\title{
Diffuse supernova neutrinos at underground laboratories
}

\author{
Cecilia Lunardini \\ Arizona State University, Tempe, AZ 85287-1504
}

\begin{abstract}
I review the physics of the Diffuse Supernova Neutrino flux (or Background, DSNB), in the context of future searches at the next generation of neutrino observatories. The theory of the DSNB is discussed in its fundamental elements, namely the cosmological rate of supernovae, neutrino production inside a core collapse supernova, redshift, and flavor oscillation effects. The current upper limits are also reviewed, and results are shown for the rates and energy distributions of the events expected at future liquid argon and liquid scintillator detectors of $\mathcal{O}(10) \mathrm{kt}$ mass, and water Cherenkov detectors up to a $0.5 \mathrm{Mt}$ mass. Perspectives are given on the significance of future observations of the DSNB, both at the discovery and precision phases, for the investigation of the physics of supernovae and of the properties of the neutrino.
\end{abstract}

Keywords: neutrinos, supernovae, neutrino detectors PACS: 14.60.Lm, 95.85.Ry, 97.60.Bw

\section{Introduction}

After a first phase of exploration, focused on solar and atmospheric neutrinos studies, neutrino physics has now entered a second phase of greater precision studies. A new generation of neutrino beam experiments is being developed to achieve a full reconstruction of the neutrino mass spectrum and mixing matrix. These experiments, primarily designed for oscillation physics, will also serve as powerful neutrino observatories: thanks to their larger detector masses and improved technologies, they will surpass their predecessors in the ability to detect and study neutrino sources of increasing distance from Earth, increasing energy, and increasing physical complexity.

The still mysterious core collapse supernovae are among these sources. After the handful of neutrino data from SN1987A, the scientific commu- 
nity is still waiting for the next detection of supernova neutrinos, to have the opportunity to learn about the physics of core collapse, to test neutrino properties, and to answer a large number of questions regarding new particles and new forces of nature. Considering that supernovae in our galaxy and its satellites are rare (1-3 per century, see e.g. $[2,3])$, it is likely that the opportunity will first be offered by the diffuse supernova neutrino flux (commonly called "diffuse supernova neutrino background", DSNB). This flux receives contributions from all the supernovae in the universe and therefore is practically constant in time ${ }^{1}$, requiring only the right experimental sensitivity to be seen. Once observed, it will turn the field of supernova neutrinos from the realm of rare events to the territory of a moderately paced and steady progress.

In addition to testing the variety of physics already probed by SN1987A - like neutrino masses and mixings, neutrino spectra formation in the star, and a number of exotica - the diffuse flux will offer other, complementary, information. Most importantly, the diffuse flux images the whole supernova population of the universe, comprised of progenitor stars of different mass and distance. Thanks to the fast rising of the supernova rate with the redshift, a substantial fraction of the DSNB at Earth originates at cosmological distances. This opens the exciting possibility to do cosmology with neutrinos, and test not only the supernova rate, but also the rate of star formation, of which supernovae are tracers.

Since the original idea that diffuse supernova neutrinos might be detectable $[4,5]$, the physics of the DSNB has matured considerably. After early upper limits that exceeded the predictions by orders of magnitude $[6,7]$, a turning point happened in 2003 when SuperKamiokande [8] placed a bound that touched the interval of existing theoretical predictions, $\Phi \sim$ $0.1-1 \mathrm{~cm}^{-2} \mathrm{~s}^{-1}$ above 19.3 of neutrino energy (see e.g.: $[10,9,11,12,13,14]$ ), thus rising the hopes that the DSNB might be detected soon. That first SuperKamiokande result, and its later updates [15, 16], have motivated more detailed theoretical predictions of the DSNB, which now include several neutrino oscillation effects $[17,18,19,20,21,22,23,24,25]$, supernova rate functions motivated by different data sets [26, 27, 28, 19, 29, 30, 21, 31], neutrino spectra from several numerical calculations [19, 32, 25, 33] and inspired

\footnotetext{
${ }^{1}$ Fluctuations in time could be seen due to individual supernovae at several megaparsecs of distance [3].
} 
by SN1987A as well [34, 21, 35, 32, 36], and even possible new non-standard physics $[37,38,39,40,41]$ and new supernova types $[30,42,43,44,31]$. Studies show that the current bound implies conditional constraints on the supernova rate $[45,43]$ and on the neutrino flux parameters $[46,15,44]$, and discuss what will be learned from a future detection on the neutrino spectra [47].

While developing the phenomenology of the DSNB, the neutrino community looks ahead to the next generation of large scale detectors $[48,49,50$, $51,52,55,56]$ of which some will expand existing projects, while others will be in completely new multi-disciplinary facilities, like the US-based Deep Underground Neutrino Experiment (DUNE) [51, 52, 53, 54].

For the new neutrino experiments, observing the DSNB is an important item of the agenda, to the point that technical upgrades are sometimes driven by this specific goal [57]. For all detection technologies backgrounds are the main limiting factors, as they often restrict the sensitive energy window considerably (to the $\sim 20-40 \mathrm{MeV}$ interval, in the case of a water Cherenkov detector). Even within the energy window, backgrounds limit the benefits of the larger detector mass.

Interestingly, searches for the DSNB show how, in the new chapter of neutrino astrophysics, what were once sought after signals - such as solar and atmospheric neutrinos - will become well known backgrounds that will have to be reduced or subtracted. This shift in the focus might have interesting implications on what characteristics might define an ideal detector several decades from now.

In this time of intense activity on the diffuse supernova neutrino flux, this review may offer a timely summary as well as a useful perspective on this new direction of research, within the activity of scoping of the next generation of neutrino observatories. The paper opens with a section of essential facts (sec. 2), which are then developed in sec. 3 for the theory and in sec. 4 for detection aspects. A section of discussion of the physics potential of the DSNB follows in closing (sec. 5).

Note: Many of the numerical results (figures and tables) that appear here were prepared specifically for this review - and not taken from previous literature - for the sake of consistency in the graphics styles and in the sets of input parameters. The original works where analogous results appear will be referenced as accurately as possible, with apologies in advance for involuntary omissions. 


\section{Diffuse supernova neutrinos: the essentials}

This section gives a minimal introduction to the subject of diffuse supernova neutrinos. It might be useful to the reader who needs only the essential information, and to others as a summary of the reminder of the review.

Stars with masses larger than $\sim 8 M_{\odot}\left(\right.$ with $M_{\odot}=1.99 \cdot 10^{30} \mathrm{Kg}$ the mass of the Sun) end their lives with the gravitational collapse of their core, followed first by neutrino emission over a time scale of about $10 \mathrm{~s}$, and then by a shock-driven, very luminous, explosion called a supernova (SN). These core collapse supernovae ${ }^{2}$ are relatively rare phenomena: their rate in the universe today (redshift $z=0$ ) is $R_{S N}(0) \sim 10^{-4} \mathrm{yr}^{-1} \mathrm{Mpc}^{-3}$. Interestingly, the supernova rate is a growing function of the redshift, $z$, signifying that supernovae were more frequent in the past (sec. 3.1).

The matter inside a supernova is dense enough (reaching nuclear density in the core, $\rho \simeq 3 \cdot 10^{14} \mathrm{~g} \mathrm{~cm}^{-3}$ ) to host a thermal population of neutrinos of all species $\left(\nu_{e}, \bar{\nu}_{e}, \nu_{\mu}, \nu_{\tau}, \bar{\nu}_{\mu}, \bar{\nu}_{\tau}\right)$ which then diffuse out and reach the Earth, carrying information on the stellar temperature in their nearly thermal energy spectrum, which peaks at $\sim 10-20 \mathrm{MeV}$. It is expected that $\nu_{e}$ and $\bar{\nu}_{e}$ have colder spectra than the other species, as they are more strongly coupled to matter (sec. 3.2). Neutrinos dominate the energetics of a supernova: they carry away about $99 \%$ of the gravitational binding energy released in the collapse, $E_{b} \simeq 3 \cdot 10^{53} \mathrm{ergs}\left(=3 \cdot 10^{46} \mathrm{~J}\right)$, which is roughly equipartitioned between the six neutrino species.

On the way between their production point and a detector on Earth, the neutrinos undergo redshift of energy and flavor conversion (oscillations), so that the flux of neutrinos (antineutrinos) of a given flavor in a detector is a linear combination of the fluxes of neutrinos (antineutrinos) originally produced in different flavors (sec. 3.3). If all supernovae are outside our immediate galactic neighborhood (farther than few megaparsecs), the flux we receive from each of them is practically infinitesimal, but the total, diffuse, flux from all supernovae combined is in principle observable. In terms of the (comoving) supernova rate, $R_{S N}(z)$, the diffuse flux of $\bar{\nu}_{e}$ in a detector at Earth, differential in energy, surface and time, is given by:

$$
\Phi_{\bar{e}}(E)=\frac{c}{H_{0}} \int_{0}^{z_{\max }} R_{S N}(z) F_{\bar{e}}\left(E^{\prime}\right) \frac{d z}{\sqrt{\Omega_{m}(1+z)^{3}+\Omega_{\Lambda}}}
$$

\footnotetext{
${ }^{2}$ Core collapse supernovae are astronomically classified as type II, Ib and Ic. Type Ia supernovae are of entirely different nature and do not involve a collapse of the stellar core.
} 
(see e.g. [19]), where $F_{\bar{e}}\left(E^{\prime}\right)$ is the contribution of an individual supernova, inclusive of neutrino oscillations and of the redshift of energy, $E^{\prime}=E(1+z)$, and differential in $E^{\prime} . \Omega_{m}$ and $\Omega_{\Lambda}$ are the fractions of the cosmic energy density in matter and dark energy respectively; $c$ is the speed of light and $H_{0}$ is the Hubble constant. $z_{\max }$ is the maximum redshift for which there is substantial star formation, $z_{\max } \sim 5$ (sec. 3.1).

Estimates (fig. 1) show that for realistic neutrino spectra and flux normalizations, the DSNB peaks around $5-7 \mathrm{MeV}$ of energy, where it can be as large as $\Phi \sim 5 \mathrm{~cm}^{-2} \mathrm{~s}^{-1} \mathrm{MeV}^{-1}$ for each neutrino species. It decays exponentially with energy above the peak. The flux in each neutrino type is typically in the range $12-20 \mathrm{~cm}^{-2} \mathrm{~s}^{-1}$ if integrated over all energies, and $\sim 0.1-0.8 \mathrm{~cm}^{-2} \mathrm{~s}^{-1}$ in the energy window of current experimental interest: $E \sim 18-35 \mathrm{MeV}$. This window is determined by backgrounds such as spallation and solar neutrinos at low energy, and atmospheric neutrinos at high energy (sec. 4.1).

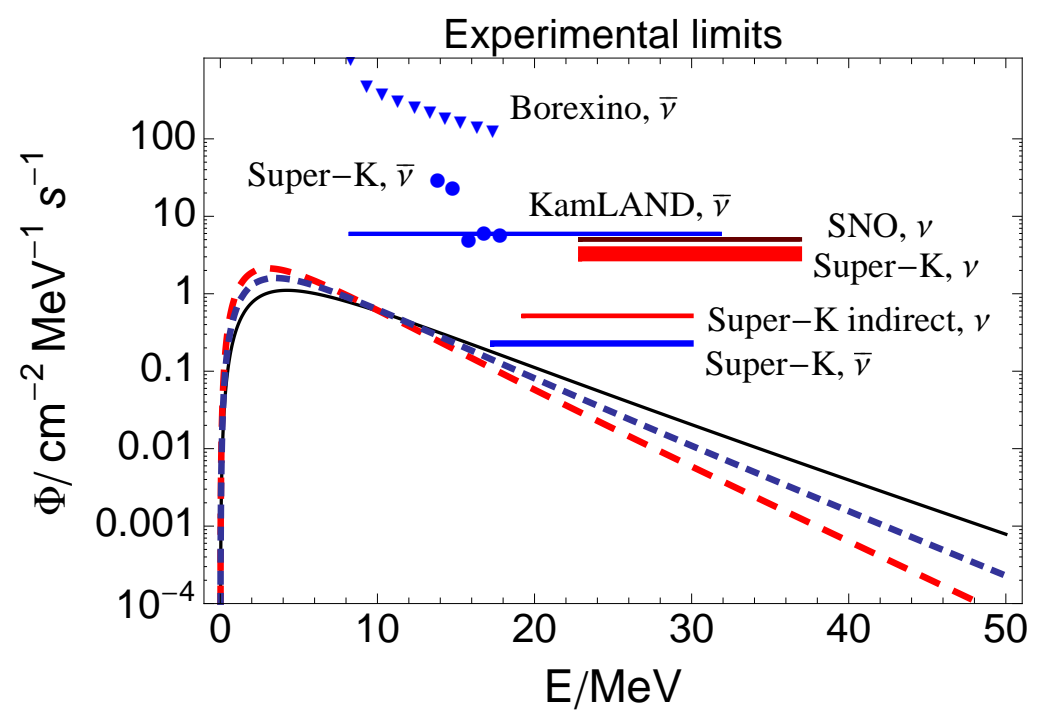

Figure 1: Experimental limits for the $\nu_{e}$ and $\bar{\nu}_{e}$ components of the DSNB, (Table 8) compared with theoretical predictions for three different examples of neutrino spectra. The upper to lower curves at $20 \mathrm{MeV}$ refer to the $\mathrm{H}, \mathrm{W}$ and $\mathrm{C}$ spectrum (Table 1), and complete flavor permutation $(p=\bar{p}=0)$, for which fluxes are maximal for the energies of interest. To make the comparison meaningful, limits on the energy-integrated fluxes have been divided by the size of the energy window of sensitivity of the experiment (see sec. 4.1). For the SK limits, the widths of the lines represent how each bound varies with the variation of the neutrino energy spectrum. See sec. 4.3 for details. 
Experimentally, many upper bounds on the DSNB exist (fig. 1; sec. 4.3). The strongest is on the $\bar{\nu}_{e}$ component, from the positron search at SuperKamiokande (SK) [15]:

$$
\Phi_{\bar{\nu}_{e}}(E>17.3 \mathrm{MeV})<2.8-3.0 \mathrm{~cm}^{-2} \mathrm{~s}^{-1} \quad \text { at } 90 \% \text { C.L. . }
$$

This limit is a factor of $\sim 2-10$ away from theoretical predictions (fig. 2), and thus suggests the detectability of the DSNB at current, or, more likely, near future detectors.

Currently, the most realistic projects for new neutrino detectors include $\mathrm{a} \sim 0.5 \mathrm{Mt}$ water Cherenkov detector [48], two $\sim 20$ kt liquid scintillator designs [55, 56] and a $\sim 40 \mathrm{kt}$ liquid argon experiment [54] (secs. 4.4-4.5). These are largely complementary in their capability for the DSNB: the Mt water Cherenkov design will have the largest statistics $(\sim 25-120$ events for a $2.5 \mathrm{Mt}$ - yr exposure, sec. 4.4), while a solution of water and Gadolinium as well as liquid scintillator allow to reduce background, and liquid argon has a unique sensitivity to electron neutrinos.

The first phase of the DSNB detection will probably be a test of the theoretically predicted event rates, and will constrain the multi-dimensional space of the parameters that govern the DSNB. A more mature phase will require high energy resolution and lower energy thresholds (compared to SK) as key elements. It would allow to reconstruct the energy spectrum of at least some flavor components of the DSNB and therefore infer information - even though in a model dependent way - on the spectrum of the neutrinos that emerge from an individual supernova (sec. 5.1). Tests of the cosmological supernova rate, $R_{S N}(z)$, will be complementary to, but probably not as sensitive as, astrophysical ones (sec. 5). Neutrino oscillation effects will be probed at a basic level, limited by the high energy thresholds and by the integration over $z$ washing out interesting spectral features (sec. 5.2). Several exotica will be probed, improving on the many strong constraints already placed by SN1987A, especially thanks to the longer propagation distances involved.

\section{The theory: diffuse neutrinos from supernovae}

\subsection{Core collapse supernovae and their cosmological rates}

How common are supernovae in the universe? The question of the rate of stellar death by core collapse is interestingly related to the question of stellar birth. Indeed, supernova progenitors have a typical life span of $\sim 10^{7}$ years, 


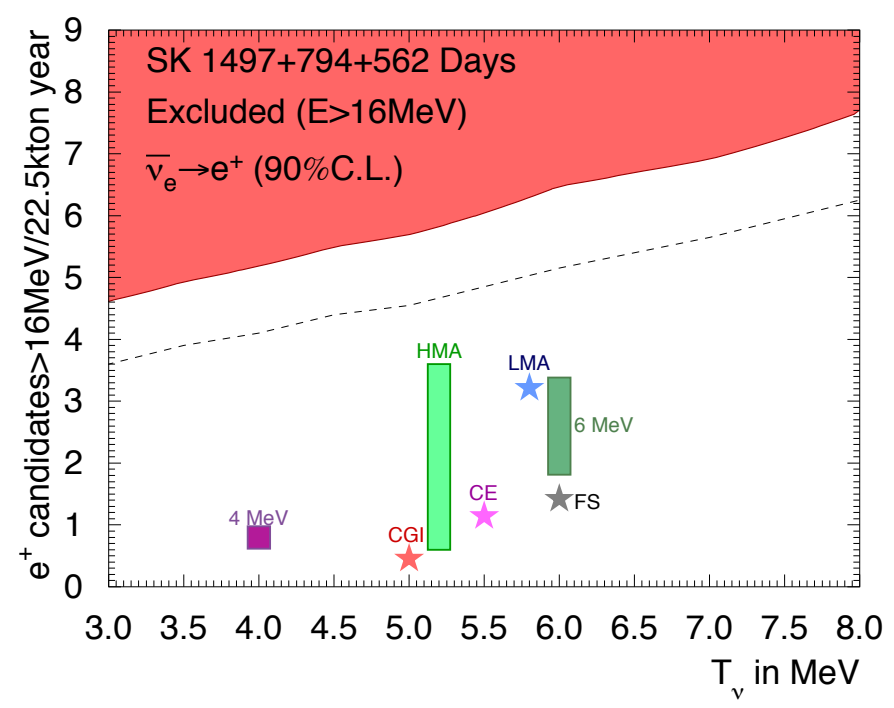

Figure 2: From [15]: The SuperKamiokande 90\% C.L. two-dimensional exclusion contour (shaded, red region) in the parameter space of event rate versus neutrino temperature (a Fermi Dirac spectrum is assumed here for the neutrinos from an individual supernova). For comparison, a number of theoretical predictions are shown. The 6 and $4 \mathrm{MeV}$ cases are from [32], and refer to Fermi-Dirac spectra. The other models use a variety of prescriptions, and are: the Cosmic Gas Infall model (CGI) [12], the Heavy Metal Abundance model (HMA) [14], the Chemical Evolution model (CE) [13], the Large Mixing Angle model [26], and Failed Supernova (FS) model [42]. For these models, the histograms represent the theoretical uncertainties. The star symbol is used for predictions with no published uncertainty. The dashed line is the one-dimensional limit obtained for each (fixed) neutrino temperature. The threshold of this analysis is $16 \mathrm{MeV}$ of positron energy (17.3 MeV of neutrino energy). 
much smaller than the characteristic time of formation of a star. Thus, it is expected that the supernova rate (SNR) as a function of the redshift $z$, should be proportional to the cosmological star formation rate (SFR), defined as the mass that forms stars per unit of (comoving) volume per unit time. Assuming that stars are are distributed in mass according to the Salpeter Initial Mass Function, $\phi(m) \propto m^{-2.35}$ [59], this proportionality between the supernova number rate $R_{S N}(z)$ and the star formation rate $R_{S F}(z)$ (which is a mass rate, note the different units) reads:

$$
R_{S N}(z)=\frac{\int_{8 M_{\odot}}^{125 M_{\odot}} d m \phi(m)}{\int_{0.5 M_{\odot}}^{125 M_{\odot}} d m m \phi(m)} R_{S F}(z) \simeq 0.014 M_{\odot}^{-1} R_{S F}(z)
$$

where a cutoff of $0.5 M_{\odot}$ has been assumed and $125 M_{\odot}$ is a tentative upper limit for the occurrence of normal core collapse supernovae (as opposed to pair instability ones or black-hole forming events) ${ }^{3}$.

The SFR is fairly well known, even though with some uncertainties, especially on its normalization. Different tracers of star formation, such as ultraviolet and far-infrared radiation, indicate that the SFR grows with $z$ (see e.g., [61] for a review). Fig. 3 shows the data and two commonly used functional fits, a piecewise one and a continuous one from [62]:

$$
\begin{aligned}
R_{S F}(z) \propto\left\{\begin{array}{cc}
(1+z)^{\beta} & z<1 \\
(1+z)^{\alpha} & 1<z<4.5 \\
(1+z)^{\gamma} & 4.5<z
\end{array}\right. \\
R_{S F}(z) \propto \frac{a+b z}{1+(z / c)^{d}}
\end{aligned}
$$

where $\alpha, \beta, \gamma, a, b, c, d$ are fit parameters. In the next sections the piecewise form will be used, for its transparency and easier comparison with other literature. Fig. 3, as well as the detailed statistical analysis in [61], show that the SFR is about $R_{S F}(0)=0.015 M_{\odot} \mathrm{yr}^{-1} \mathrm{Mpc}^{-3}$ today, grows with power $\beta \simeq 3$ ( $\beta=3.28$ best fit) up to $z \simeq 1$, flattens at larger redshift $(\alpha=-0.26$ best fit) and decreases at $z \gtrsim 4.5(\gamma=-7.8$ best fit $)$.

\footnotetext{
${ }^{3}$ Although generally motivated by stellar evolution studies (see, e.g. [60]), the integration limits in eq. (3), are not robustly known. The resulting uncertainty on the supernova rate is subdominant compared to other major uncertainties of a factor $\sim 2$ that are discussed in this section, see fig. 4 and 5 .
} 

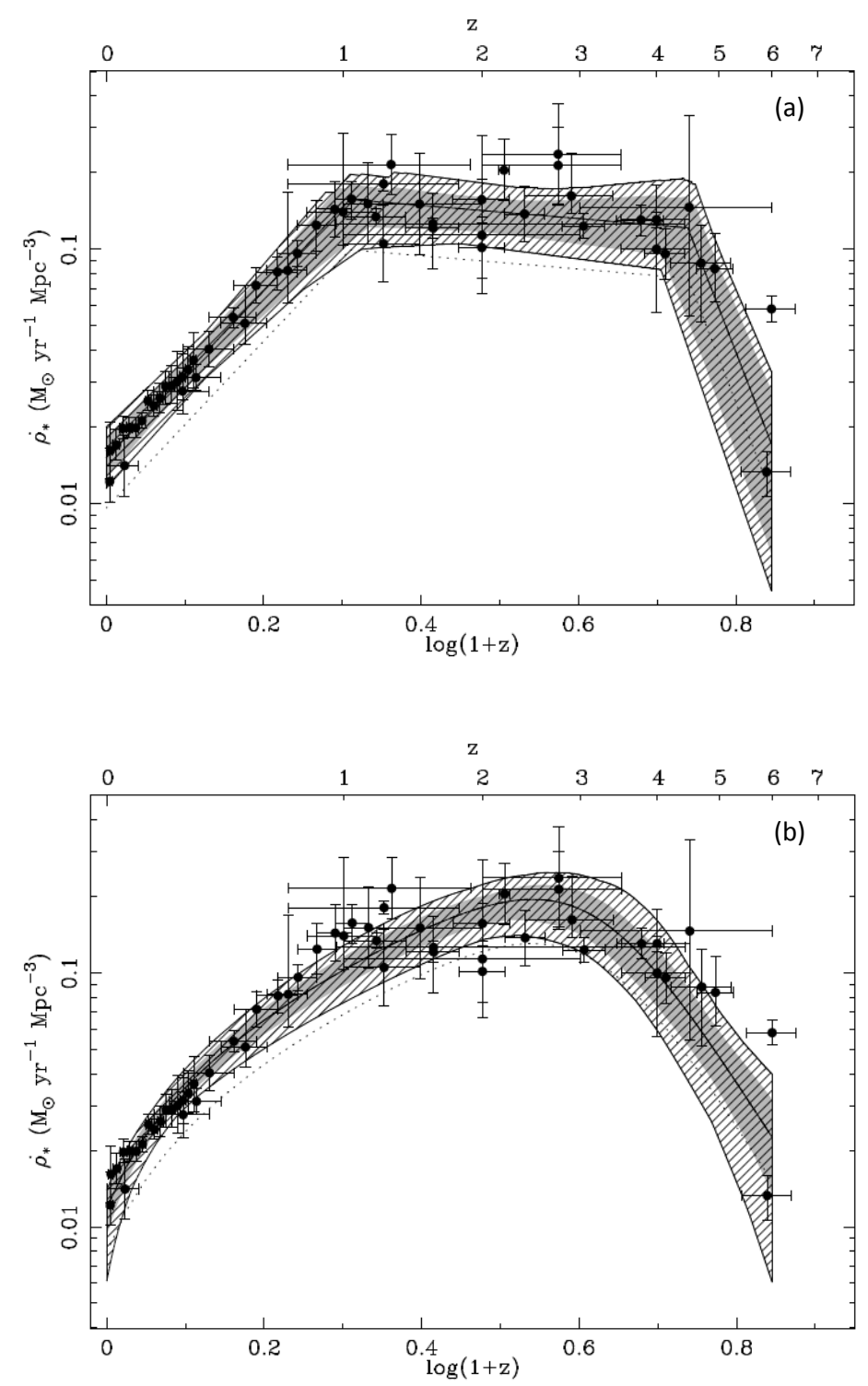

Figure 3: Measurements of the cosmological star formation rate and functional fits, from [61]. The grey shaded and hatched regions are the $1 \sigma$ and $3 \sigma$ confidence regions obtained with (a) the piecewise parameterization and (b) the Cole et al. parameterization, Eq. (4). The SK limit on the DSNB, Eq. (2), constrains the normalization to values lower by a factor $0.74-1$ depending on the neutrino spectrum; the rescaled SFR is indicated by the dotted curves, see [61] for details. 


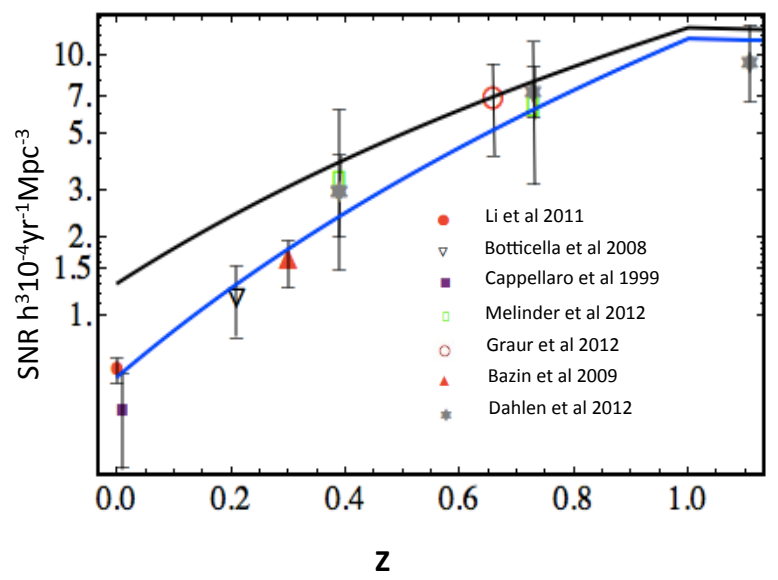

Figure 4: Direct supernova rate measurements as of 2013 [63, 64, 65, 66, 67, 68, 69]; from $[70,71]$, with their best fit function (lower curve). The flattening of the function at $z \simeq 1$ is motivated by SFR observations (fig. 3) and not by the supernova data themselves. The upper curve represents Eq. (5) and is the SNR obtained from a SFR data fit via Eq. (3) $[61]$.

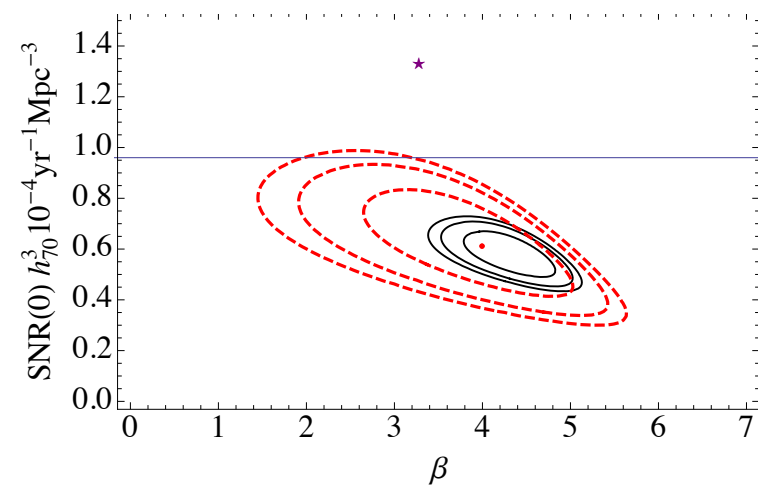

Figure 5: Best fit point and isocontours of $\chi^{2}$ in the space of the parameters describing the SNR function, $R_{S N}(z)[70,71]$. These are the intercept, $R_{S N}(0)$ (in units of $10^{-4} \mathrm{yr}^{-1} \mathrm{Mpc}^{-3}$ ) and the power, $\beta$ (for the piecewise parameterization in Eq. (4)). Results are presented for two different analyses, with statistical errors only (solid, black curves), and with statistical and correlated systematic errors (dashed regions, in red). For each, the contours refer to $68.3,90,95.4 \%$ C.L.. The star represents the star formation rate-favored parameters, $\left(\beta, R_{S N}(0)\right)=(3.28,1.33)$, [61]. The horizontal line is a lower bound from Smarrtt et al. [72]. 
The corresponding best fit SNR, obtained via Eq. (3), can be written as:

$$
R_{S N}(z)=R_{-4} 10^{-4} \mathrm{yr}^{-1} \mathrm{Mpc}^{-3}\left\{\begin{array}{cc}
(1+z)^{3.28} & z<1 \\
(1+z)^{-0.26} & 1<z<4.5 \\
(1+z)^{-7.8} & 4.5<z
\end{array}\right.
$$

with $R_{-4} \sim 1$ describing the uncertainty in the normalization. It is plotted in fig. 4 (left panel, upper dashed line).

Fig. 4 also shows the direct measurements of the SNR as of 2013 [63, 64, $65,66,67,68,69]$ and their piecewise fitting curve [70, 71]. For the same fit, fig. 5 gives the allowed region of the parameters $R_{S N}(0)$ and $\beta$; the correlation between the two quantities is evident in the figure. The comparison between the SNR obtained directly and that derived from the SFR (upper curve in

fig. 4) shows a discrepancy at low redshift, which can be interpreted as a measure of the systematic errors involved. At least part of this discrepancy is resolved when including faint or completely dark supernovae [73, 31].

\subsection{Neutrinos from supernovae}

Core collapse supernovae are the only site in the universe today where the matter density is large enough to have the buildup of a thermal gas of neutrinos. Thanks to their lack of electromagnetic interaction, these neutrinos can diffuse out of the star over a time scale of few seconds, much shorter than the diffusion time of photons. This makes the neutrinos the principal channel of emission of the $\mathcal{O}\left(10^{53}\right)$ ergs of gravitational energy that is liberated in the collapse. The energy spectrum of neutrinos of each flavor is expected to be thermal near the surface of decoupling from matter ("neutrinosphere"), but then it changes due to propagation effects. One of these effects is scattering. Numerical modeling indicates that, after scattering right outside the decoupling region, neutrinos of a given flavor $w(w=e, \mu, \tau)$ have energy spectrum:

$$
\frac{d N_{w}}{d E} \simeq \frac{\left(1+\alpha_{w}\right)^{1+\alpha_{w}} L_{w}}{\Gamma\left(1+\alpha_{w}\right) E_{0 w}^{2}}\left(\frac{E}{E_{0 w}}\right)^{\alpha_{w}} e^{-\left(1+\alpha_{w}\right) E / E_{0 w}},
$$

[74], where $E$ is the neutrino energy, $L_{w}$ is the energy emitted in the species $w$ and $E_{0 w}$ is the average energy of the spectrum. The quantity $\alpha_{w}$ is a numerical parameter, $\alpha_{w} \sim 2-5$ [74], describing the shape of the spectrum. Considering typical temperatures of matter near the collapsed core, one expects average energies in the $10-20 \mathrm{MeV}$ range. The non-electron neutrino 
flavors, $\nu_{\mu}, \nu_{\tau}, \bar{\nu}_{\mu}$ and $\bar{\nu}_{\tau}$ (each of them denoted as $\nu_{x}$ from here on ${ }^{4}$ ), interact with matter more weakly than $\nu_{e}$ and $\bar{\nu}_{e}$ (via neutral current processes only), and therefore decouple from matter in a denser and hotter region. Additionally, an asymmetry exist in the coupling with matter of $\nu_{e}$ and $\bar{\nu}_{e}$ : these are kept in thermal equilibrium by charged current interaction on neutrons and protons respectively, and the overabundance of neutrons relative to protons implies a lower decoupling temperature for $\nu_{e}$. Therefore, we expect a hierarchy of average energies:

$$
E_{0 e}<E_{0 \bar{e}}<E_{0 x}
$$

\begin{tabular}{|c|c|c|c|c|c|c|}
\hline \hline Case & $E_{0 e}(\mathrm{MeV})$ & $E_{0 \bar{e}}(\mathrm{MeV})$ & $E_{0 x}(\mathrm{MeV})$ & $\alpha_{e}$ & $\alpha_{\bar{e}}$ & $\alpha_{x}$ \\
\hline \hline Cold $(\mathrm{C})$ & 9 & 11 & 13 & 3 & 3 & 2 \\
\hline Warm $(\mathrm{W})$ & 11 & 14 & 15 & 3 & 3 & 2 \\
\hline Hot $(\mathrm{H})$ & 12 & 15 & 18 & 3 & 3 & 2 \\
\hline \hline
\end{tabular}

Table 1: The set of spectral parameters used here. Their labels, Hot, Warm and Cold, are purely conventional. The total energies emitted in each flavor are $L_{e}=L_{\bar{e}}=L_{x}=5 \times 10^{52}$ ergs in all cases.

While the basic spectral features and the total energy emitted in neutrinos are generic predictions, a detailed study of neutrino emission in a supernova requires complex numerical calculations, that have been performed by several groups in the quest of reproducing the observed explosion that follows the collapse (see e.g. [78, 79] for a review).

Here the form (6) will be used for the spectrum of each neutrino flavor before oscillations, integrated over the $\sim 10$ s duration of the neutrino burst. For illustration, three different sets of spectral parameters are used, conventionally named Hot $(\mathrm{H})$, Warm (W) and Cold (C). They are summarized in Table 1. They represent examples, loosely covering the range of parameters that have emerged from recent numerical simulations (e.g., $[80,81,82,83,84,85,86])$. The $\mathrm{C}$ case is quantitatively close to the results of the Basel group [83] (after time-integration), that were calculated over

\footnotetext{
${ }^{4}$ For the energies of interest here, the produced fluxes of $\nu_{\mu}, \nu_{\tau}$ are equal with very good approximation. They are also nearly equal to the fluxes of $\bar{\nu}_{\mu}$ and $\bar{\nu}_{\tau}$, up to corrections due to weak magnetism [75].
} 
several seconds after the collapse. The $\mathrm{W}$ case is meant to represent the situation in which the spectra of $\bar{\nu}_{e}$ and $\nu_{x}$ quickly become similar immediately after $\sim 1 \mathrm{~s}$ accretion phase (see e.g., [82]). The $\mathrm{H}$ case account for the possibility of more energetic neutrino spectra, that might be realized in a star with a longer accretion phase (see e.g., [86]).
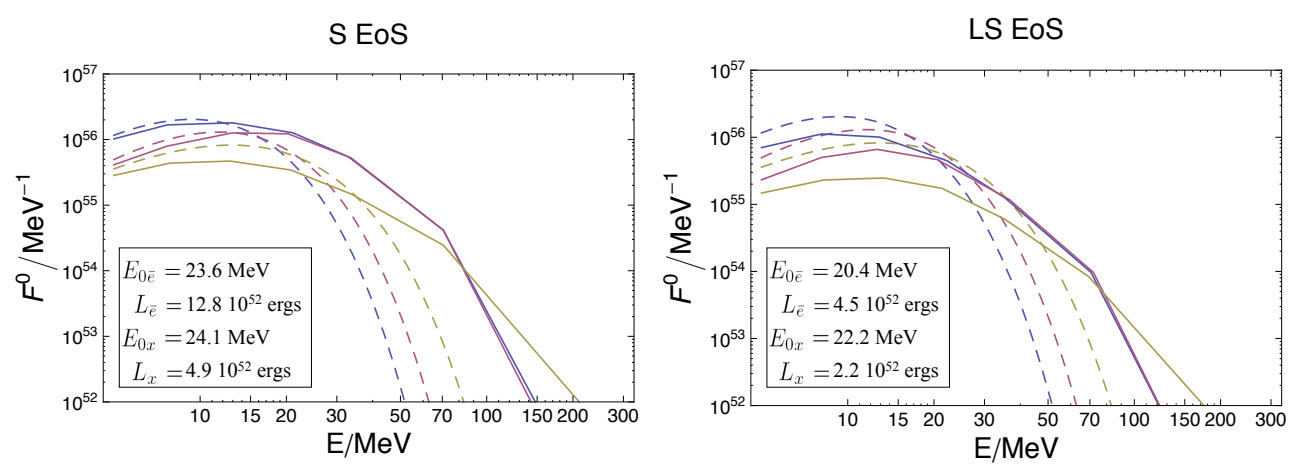

Figure 6: From [42]: neutrino fluxes at production inside the star for direct black holeforming collapse (solid lines), taken from the numerical calculation of [87]. Curves from upper to lower at $5 \mathrm{MeV}$ correspond to $\nu_{e}, \bar{\nu}_{e}, \nu_{x}$. Spectra are shown for the Shen et al. (left panel) and Lattimer-Swesty (right) equation of state. For each, the neutrino average energies and the total energy emitted per flavor are given (inserts). See text for details. The dashed lines represent typical spectra for neutron star-forming collapse (Eq. (1)), with the parameters $E_{0 \bar{e}}=15 \mathrm{MeV}, E_{0 x}=18 \mathrm{MeV}, L_{\bar{e}}=L_{x}=5 \cdot 10^{52}$ ergs, $\alpha_{\bar{e}}=3.5$ and $\alpha_{x}=2.5$.

The examples of spectra above refer to the most common scenario for a supernova: a collapse that leads to the formation of a neutron star ("neutron star-forming collapses", NSFCs). Detailed studies have appeared $[88,84,89,90,91,87]$. on the rarer case of direct collapse into a black hole without explosion, i.e., a failed supernova. This is the fate of stars with $M \gtrsim$ $25-40 M_{\odot}$, which comprise a $9-22 \%$ fraction of all supernova progenitors [42]. It was shown that the neutrino emission from these direct black holeforming collapses (DBHFCs) is somewhat more luminous and decidedly more energetic than for neutron star-forming collapses - depending on the equation of state (EoS) of nuclear matter - due to the rapid contraction of the newly formed protoneutron star preceding the black hole formation. This suggests that the hotter contribution of black hole-forming collapses to the DSNB might exceed that of neutron star-forming ones in part of the energy spectrum $[42,43,92,44]$. 
Fig. 6 shows the neutrino fluxes emitted in a direct black hole-forming collapse, from [87]. They were obtained for the $40 M_{\odot}$ progenitor in [93] with the stiffer Shen et al. (S) EoS [94] (incompressibility $K=281 \mathrm{MeV}$ ) and the softer Lattimer-Swesty (LS) one [95] (with $K=180 \mathrm{MeV}$, see [87]). It appears that average energies are $E_{0} \sim 20-24 \mathrm{MeV}$ for all neutrino flavors, with a stiffer EoS corresponding to more energetic neutrino spectra. Notice how the $\nu_{e}$ and $\bar{\nu}_{e}$ components are especially luminous due to the high rate of capture of electrons and positrons on nuclei.

\subsection{Neutrino flavor conversion}

For a long time a mere hypothesis, neutrino flavor conversion is now a reality well established experimentally [96, 1]. The leading mechanism driving conversion is neutrino oscillations due to the neutrino being massive and having non-zero mixing between the neutrino mass eigenstates, $\nu_{i}(i=$ $1,2,3)$ with masses $m_{i}$, and the flavor eigenstates, $\nu_{\alpha}(\alpha=e, \mu, \tau): \nu_{\alpha}=$ $\sum_{i} U_{\alpha i} \nu_{i}$. The standard parameterization of the mixing matrix (see e.g. [97]) is in terms of three mixing angles: $\theta_{12}, \theta_{23}, \theta_{13}$, and a complex phase, $\delta$. Oscillation effects depend on these parameters, and on the mass squared differences, $\Delta m_{i j}^{2}=m_{i}^{2}-m_{j}^{2}$, as well as on the neutrino energy and on the density and composition of the medium of propagation due to forward scattering on the matter constituents (refraction).

After an intense phase of neutrino experiments on solar, atmospheric, reactor and accelerator neutrinos, measurements are available for $\theta_{12}, \theta_{13}, \theta_{23}, \Delta m_{21}^{2}$ and $\left|\Delta m_{31}^{2}\right|$ (see e.g., [98] and references therein):

$$
\begin{array}{r}
\left|\Delta m_{31}^{2}\right|=\left(2.43_{-0.10}^{+0.06}\right) \cdot 10^{-3} \mathrm{eV}^{2}, \quad \Delta m_{21}^{2}=\left(7.54_{-0.22}^{+0.26}\right) \cdot 10^{-5} \mathrm{eV}^{2}, \\
\sin ^{2} \theta_{12}=0.307_{-0.016}^{+0.018}, \quad \sin ^{2} \theta_{23}=0.386_{-0.021}^{+0.0244}, \quad \sin ^{2} \theta_{13}=0.0241 \pm 0.0025 .
\end{array}
$$

The phase $\delta$ currently remains unmeasured; it has a subdominant or negligible effect on supernova neutrinos [99, 100], and therefore it will be set to zero from now on. Current data are not sufficient to distinguish the sign of $\Delta m_{31}^{2}$ : The two possibilities, $\Delta m_{32}^{2} \approx \Delta m_{31}^{2}>0$ and $\Delta m_{32}^{2} \approx \Delta m_{31}^{2}<0$, are referred to as normal and inverted mass hierarchy/ordering ${ }^{5}$.

\footnotetext{
${ }^{5}$ The parameter values in eq. (8) refer to the normal mass hierarchy; the corresponding numbers for inverted hierarchy are only minimally different, and consistent within the error with those in eq. (8). See [98] for details.
} 
With these information, it has been possible to study flavor conversion of supernova neutrinos in detail, uncovering a very rich pattern. One can distinguish four spatially separated stages of conversion, that contribute to the final $\nu_{e}$ and $\bar{\nu}_{e}$ survival probabilities (Table 2):

- Within $\sim 200 \mathrm{Km}$ radius in the star, collective flavor oscillations occur due to neutrino-neutrino coherent scattering $[101,102]^{6}$ when the density of neutrinos exceeds that of electrons [104]. This should be the case $\sim 1-5 \mathrm{~s}$ after the core bounce. The most common effect observed in numerical simulations is a swap of the spectra of the electron and non-electron neutrinos and antineutrinos for the inverted mass hierarchy and above a certain critical energy, $E_{c} . E_{c}$ is small (below typical detection thresholds) for antineutrinos, while for neutrinos it depends on the fluxes of $\nu_{e}$, and $\bar{\nu}_{e}$ and $\nu_{x}\left(\nu_{x}=\nu_{\mu}, \nu_{\tau}, \bar{\nu}_{\mu}, \bar{\nu}_{\tau}\right)$ as:

$$
\int_{E_{c}}^{\infty}\left(F_{e}^{0}-F_{x}^{0}\right)=\int_{0}^{\infty}\left(F_{\bar{e}}^{0}-F_{x}^{0}\right),
$$

with $E_{c} \simeq 3-10 \mathrm{MeV}$ as typical values [105]. Here I denote as $P_{c}$ and $\bar{P}_{c}$, the step-like $\nu_{e}$ and $\bar{\nu}_{e}$ survival probabilities after collective effects. They vary over the duration of the neutrino burst, due to possible suppression of collective effects in the accretion phase [106, 107, 108], and to the time variation of the fluxes in eq. (9).

- At larger radii, where neutrino-neutrino coherent scattering is negligible, conversion is driven by coherent scattering on electrons, according to the Mikheev-Smirnov-Wolfenstein effect (MSW) [109, 110], that was first elaborated in the context of solar neutrino and then applied to supernova neutrinos [111]. In essence, the MSW effect is the idea that a small neutrino mixing induces more than $50 \%$ change of flavor if neutrinos propagate in matter and:

(i) the matter density along the neutrino trajectory varies slowly enough that there are no quantum transitions between different eigenstates of the Hamiltonian (adiabatic propagation). Under this condition, a neutrino that is produced in given eigenstate of the Hamiltonian in matter, $\nu_{i, m}$ will remain in such state while the flavor composition of the state

\footnotetext{
${ }^{6}$ See e.g., [103] for a comprehensive review of the vast literature on this topic.
} 
itself varies as an effect of the varying matter density along the neutrino trajectory. At emergence from the star, the neutrino is in the vacuum state $\nu_{i}$, whose flavor composition is described by the mixing matrix. The process results in a strong change of flavor if

(ii) the neutrinos cross a region where the density is such (resonance density) that a cancellation occurs between the matter and kinetic terms of the Hamiltonian, thus producing a resonant behavior.
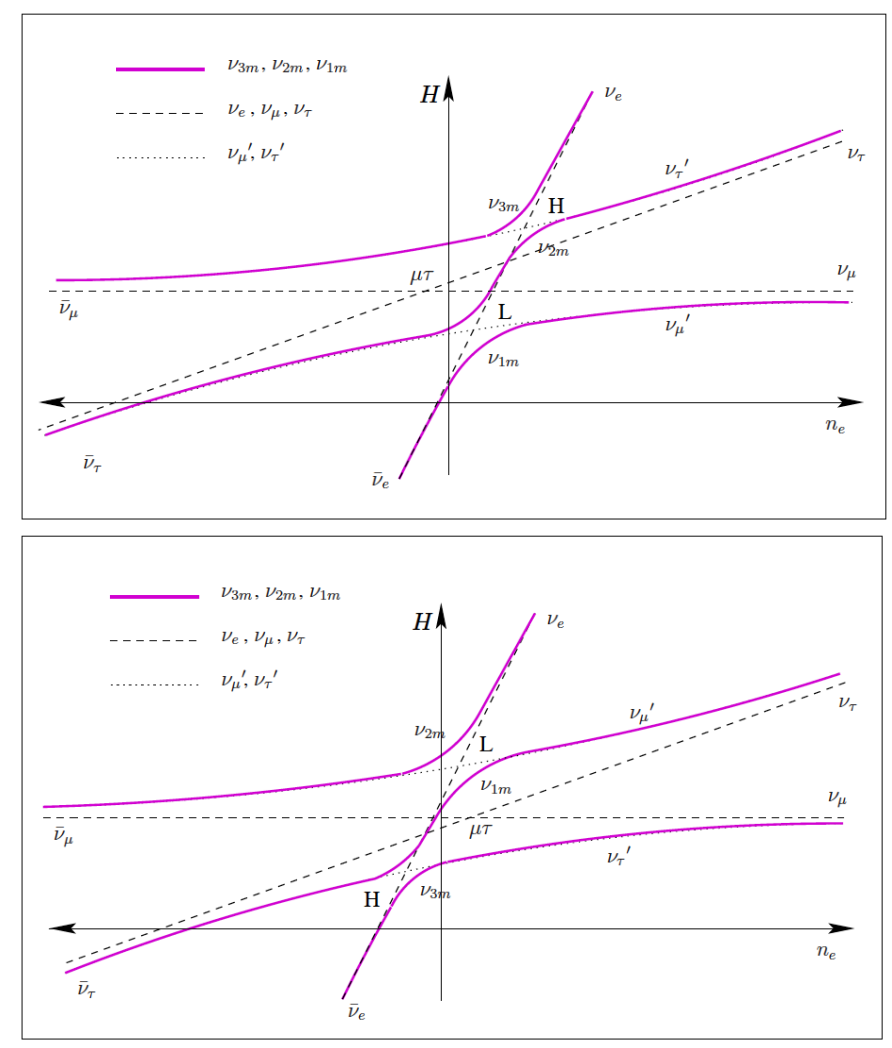

Figure 7: Schematic representation of the neutrino energy levels in matter vs electron number density, $n_{e}$, for normal (upper pane) and inverted (lower pane) mass hierarchy, from [99]. Dashed line: in the absence of mixing (unphysical), solid line: with mixing (physical case). The semi-plane with positive (negative) density, $n_{e}>0\left(n_{e}<0\right)$, describes the conversion of neutrinos (antineutrinos), to account for the fact that the refraction potential has opposite sign for neutrinos and antineutrinos. The positions of the high $(\mathrm{H})$ and low (L) density MSW resonances are marked. See [99, 112] for details.

Two MSW resonances relevant for $\nu_{e}$ and $\bar{\nu}_{e}$ conversion are realized inside a supernova, corresponding to the two independent mass squared 
splittings (fig. 7). The first resonance occurs at $\rho \sim 10^{3} \mathrm{~g} \cdot \mathrm{cm}^{-3}$. For a power-law profile

$$
\rho(r)=10^{13} C\left(\frac{10 \mathrm{~km}}{r}\right)^{3} \mathrm{~g} \cdot \mathrm{cm}^{-3} \quad C \simeq 1-15
$$

[112], that applies before the shock wave reaches the resonance layer [113], this resonance is adiabatic [114]. it depends on the neutrino mass hierarchy: it affects neutrinos (antineutrinos) if the mass hierarchy is normal (inverted).

- The second resonance is at $\rho \sim 10 \mathrm{~g} \cdot \mathrm{cm}^{-3}$ and is described by the parameters $\theta_{12}$ and $\Delta m_{21}^{2}$, known as the LMA (Large Mixing Angle MSW) solution of the solar neutrino problem. The resonance is adiabatic and it affects neutrinos. Antineutrinos undergo non-resonant conversion, thanks to $\theta_{12}$ being large.

- A fourth stage of oscillations happens inside the Earth, where the physical conditions resemble those of the second resonance. While potentially important for neutrinos from a galactic supernova, these oscillations affect the DSNB by less than few per cent [115], so they will not be discussed here.

The fluxes of $\nu_{e}$ and $\bar{\nu}_{e}$ after oscillations are a mixture of the original fluxes in these flavors and of the original muon and tau component. Up to a geometric factor due to the distance from the star (omitted here for simplicity), they are described by the permutation parameters $p, \bar{p}$ as:

$$
\begin{aligned}
& F_{e}=p F_{e}^{0}+(1-p) F_{x}^{0}, \\
& F_{\bar{e}}=\bar{p} F_{\bar{e}}^{0}+(1-\bar{p}) F_{x}^{0} .
\end{aligned}
$$

The oscillated fluxes, $F_{e}, F_{\bar{e}}$, are more energetic than the original ones due to the higher average energy of $F_{x}^{0}$. This spectral hardening is one of the main signature of neutrino flavor conversion in a supernova. The calculation of $p$ and $\bar{p}$ is at times complex, and for this we refer to the literature (e.g., $[114,112,116,117])$. Here the results are shown in Table 2.

It appears that, considering $0 \leq P_{c}, \bar{P}_{c} \leq 1, p$ and $\bar{p}$ are in the ranges:

$$
\begin{aligned}
& p=0-\sin ^{2} \theta_{12} \simeq 0-0.32, \\
& \bar{p}=0-\cos ^{2} \theta_{12} \simeq 0-0.68 .
\end{aligned}
$$




\begin{tabular}{ccl}
\hline & Normal hierarchy & Inverted hierarchy \\
\hline$p$ & $\sin ^{2} \theta_{12}\left(1-P_{c}\right)$ & $\sin ^{2} \theta_{12} P_{c}$ \\
$\bar{p}$ & $\cos ^{2} \theta_{12} \bar{P}_{c}$ & $\cos ^{2} \theta_{12}\left(1-\bar{P}_{c}\right)$ \\
\hline
\end{tabular}

Table 2: The electron neutrino (antineutrino) survival probability, $p(\bar{p})$ for neutrino emerging from a supernova. Here $P_{c}$ and $\bar{P}_{c}$ are the step-like survival probabilities after collective effects.

In general, both quantities vary with the neutrino energy and with time, via the functions $P_{c}$ and $\bar{P}_{c}$, and also due to a change in the adiabaticity of the high density resonance due to shockwave effects [17, 23]. However, for the purpose of the DSNB calculation, $p$ and $\bar{p}$ can typically be taken as constant in energy. This is justified if the critical energy $E_{c}$, is below the energy window for detection of the diffuse flux. Furthermore, integrating over the duration of the burst and over the supernova population smears out the energy dependence of the oscillation effects $[24,25]$.

As a cautionary remark, it should be noted that the phenomenology of oscillations induced by neutrino-neutrino scattering is still in the phase of initial exploration, and therefore the results in Table 2 may change as studies progress. For example, recently it has been understood that multiple spectral swaps due to neutrino-neutrino scattering can occur [118], and that a richer pattern of spectral splits may emerge from including the effect of the so called neutrino "halo" $[119,120,121]$, and the breaking of the azimuthal asymmetry of the collective oscillations $[122,123,124]$. Therefore the expressions in Table 2 may not be entirely applicable to the DSNB. Variations between individual supernovae (e.g., in the matter density profile, see [87]) may further complicate the description of conversion effects for the DSNB. In view of these uncertainties, it is adequate and convenient - for the sake of generality - to describe the effects of oscillations on the DSNB in terms of $p$ and $\bar{p}$ intended as averaged over time and over the supernova population. I neglect their energy dependence for the reasons discussed so far, and vary them in the numerical intervals (13), which are guaranteed to be valid under the sole conditions of adiabaticity of the lower density resonance and absence of turbulence (see e.g. [125]).

\subsection{Constraints from SN1987A}

It is interesting to check how predictions compare with the only data we currently have on supernova neutrinos - those from SN1987A - and what 

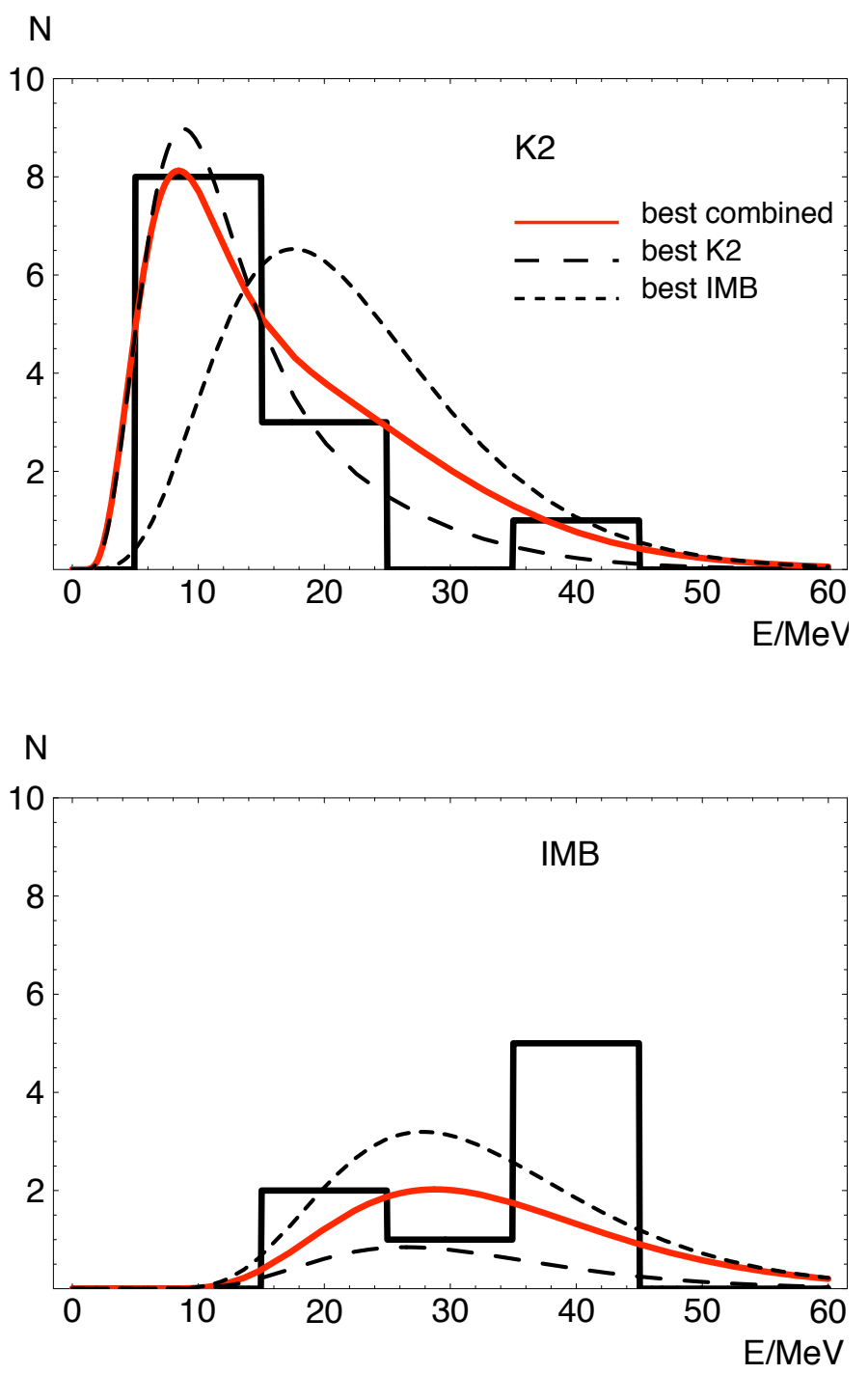

Figure 8: The observed positron energy spectra of events at Kamiokande II (K2) and IMB, compared with the predicted spectra in the points of minimum $\chi^{2}$ for the two data sets saparately and combined [21]. 
these data alone tell us without theoretical priors [34, 21, 36]. Fig. 8 shows the data published by the Kamiokande-II and IMB experiments [126, 127] and the best fits from a maximum likelihood analysis of the two datasets separately and combined [21]. The analysis was carried out assuming that all the events were from inverse beta decay $\left(\bar{\nu}_{e}+p \rightarrow n+e^{+}\right)$, and with five fit parameters: $L_{\bar{e}}, L_{x}, E_{0 \bar{e}}, E_{0 x}$ and $\bar{p}$ (the latter was constrained to be in the range in eq. (13)). For simplicity, $\alpha_{\bar{e}}=\alpha_{x}=2.3$ (which give a spectral shape close to Fermi-Dirac) was held fixed. The figure shows that the two datasets are compatible, in spite of the tension in their favoring different neutrino energy spectra. The best fit of all the data together is realized for maximum permutation of fluxes $(\bar{p}=0.68)$ and:

$$
\begin{array}{ll}
E_{0 \bar{e}}=4.2 \mathrm{MeV} & L_{\bar{e}}=4.4 \cdot 10^{53} \mathrm{ergs} \\
E_{0 x}=14.9 \mathrm{MeV} & L_{x}=0.8 \cdot 10^{53} \mathrm{ergs},
\end{array}
$$

where the $\bar{\nu}_{e}$ parameters are somewhat in tension with the theoretical expectations for the unusually low average energy and the very large total energy $L_{\bar{e}}$.

The region of the parameter space allowed at different confidence levels is large and is shown in fig. 9, where the hierarchy $E_{0 \bar{e}} \leq E_{0 x}$ (Eq. (7)) has been imposed. A comparison between this figure and Table 1 shows that the data are compatible with numerical predictions (only marginally for the $\mathrm{H}$ spectrum), but at the same time allow much softer neutrino spectra, which thus remain a possibility on the basis of data alone. How these softer spectra impact the DSNB is discussed in sec. 3.5.4.

\subsection{Diffuse flux at Earth}

\subsubsection{Generalities}

Armed with the ingredients discussed so far, one can calculate the DSNB expected at Earth, using Eq. (1). Here I first discuss a scenario in which a number of approximations and simplifying assumptions hold:

- all the neutrinos are from neutron star-forming collapses (Table 1)

- the neutrino emission is identical for all neutron star-forming progenitors, which is a condition for the validity of Eq. (1). The generalization to a mixed population (neutron star-forming and black hole-forming) will be discussed in sec. 3.5.5. 


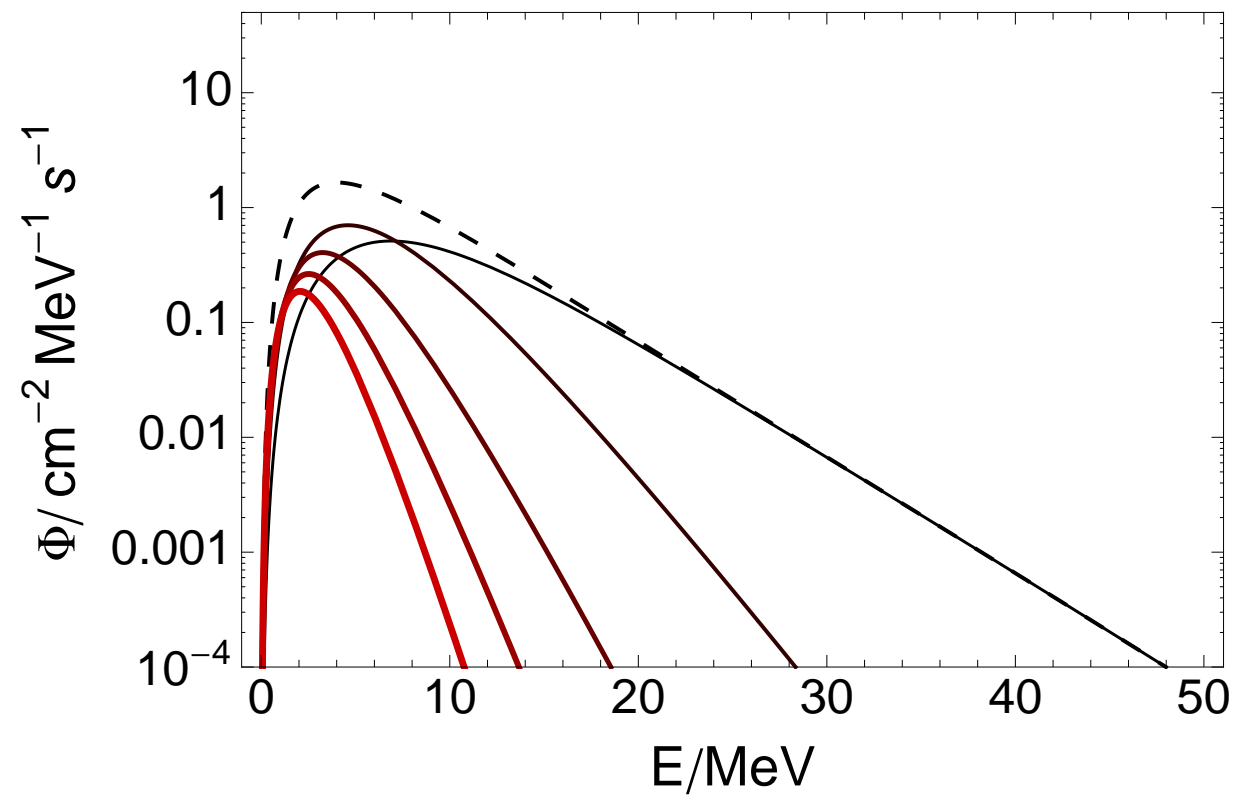

Figure 9: From [21]: projections of the $68 \%, 90 \%, 99 \%$ C.L. regions allowed by the SN1987A data on the planes $E_{0 \bar{e}}-E_{0 x}$ and $E_{0 x}-L_{x}$, with the hierarchy $E_{0 \bar{e}}<E_{0 x}$ imposed as a prior. The dots in each panel mark the projections of the points of maximum likelihood. The entire plane $E_{0 \bar{e}}-L_{\bar{e}}$ (not shown) is allowed at $68 \%$ C.L.. 
- the total energy emitted in neutrinos is fixed and equipartitioned among the flavors: $L_{e}=L_{\bar{e}}=L_{x}=0.5 \cdot 10^{53}$ ergs.

- the piecewise form of the SNR, Eq. (5), is used, with $R_{-4}=1 .^{7}$

- the survival probabilities $p, \bar{p}$ are constant in energy, which is valid in several representative cases (sec. 3.3).

- due to backgrounds, the energy thresholds that are considered realistic for the detection of the DSNB are $E_{t h} \simeq 11-20 \mathrm{MeV}$ (see sec. 4). These will be the focus of the discussion.

Under these conditions, one can write the $\nu_{e}, \bar{\nu}_{e}$ component of the DSNB as:

$$
\begin{aligned}
& \Phi_{e}(E)=p \Phi_{e}^{0}(E)+(1-p) \Phi_{x}^{0}(E), \\
& \Phi_{\bar{e}}(E)=\bar{p} \Phi_{\bar{e}}^{0}(E)+(1-\bar{p}) \Phi_{x}^{0}(E),
\end{aligned}
$$

where

$$
\Phi_{w}^{0}(E)=\frac{c}{H_{0}} \int_{0}^{z_{\max }} R_{S N}(z) F_{w}^{0}\left(E^{\prime}\right) \frac{d z}{\sqrt{\Omega_{m}(1+z)^{3}+\Omega_{\Lambda}}}
$$

is the component of the DSNB of the species $w$ in absence of oscillations and $E^{\prime}=E(1+z)$ (sec. 3.3). While not directly observable, $\Phi_{e}^{0}, \Phi_{\bar{e}}^{0}, \Phi_{x}^{0}$ are useful to understand the main features of the DSNB and to calculate results for several different oscillations scenarios.

For applications, it is interesting to study the integrated fluxes (oscillated and unoscillated) above a threshold $E_{t h}$ :

$$
\phi_{w}\left(E_{t h}\right)=\int_{E_{t h}}^{\infty} \Phi_{w}(E) d E, \quad \phi_{w}^{0}\left(E_{t h}\right)=\int_{E_{t h}}^{\infty} \Phi_{w}^{0}(E) d E .
$$

\footnotetext{
${ }^{7}$ Results can be rescaled to reproduce those of other literature, e.g. [19] $\left(R_{-4}=0.87\right)$, $[21]\left(R_{-4}=0.67\right.$, best fit $)$ and $[61]\left(R_{-4}=1.84\right)$.
} 


\subsubsection{Dependence on the original neutrino spectrum}

Here I describe how the neutrino fluxes at production, $F_{w}^{0}$, influence the DSNB. For simplicity, I consider the unoscillated fluxes $\Phi_{w}^{0}$ and $\phi_{w}^{0}$; as their features apply to the oscillated ones through the combination (15).

The integral in Eq. (16) can not be calculated exactly, and so one can resort to certain approximations or to numerical calculation. The following approximation is valid at high energy $(E \gtrsim 15-20 \mathrm{MeV})$ [128]:

$$
\Phi_{w}^{0} \simeq R_{S N}(0) \frac{c}{H_{0}} \frac{L_{w}}{\Gamma\left(2+\alpha_{w}\right) \epsilon_{w}^{2}} e^{-\frac{E}{\epsilon_{w}}} \sum_{k=0}^{\eta_{w}}\left[\left(\frac{E}{\epsilon_{w}}\right)^{\alpha_{w}-1-k} \frac{\eta_{w} !}{\left(\eta_{w}-k\right) !}\right]
$$

where $\Gamma$ stands for the Gamma function, $\eta_{w} \equiv \alpha_{w}+\beta-3 \Omega_{m} / 2(\beta$ is the power of growth of the SFR and SNR with $z$, Eq. (4)) and $\epsilon=E_{0 w} /\left(1+\alpha_{w}\right)$. The dependence on the parameters $\alpha$ and $\gamma$ of the SFR is neglected. The expression (18) exceeds the exact result by up to $40 \%$ above $19 \mathrm{MeV}$ of energy. It has also been observed $[129,128]$ that a simple exponential form, $\Phi_{w}^{0}=\Phi_{w}^{0}(0) e^{-E /\langle E\rangle_{w}}$ (with $\langle E\rangle_{w} \sim \epsilon_{w}$ ) is adequate for realistic parameters and for $E \gg\langle E\rangle_{w}$. In what follows, exact, numerically calculated results will be discussed.
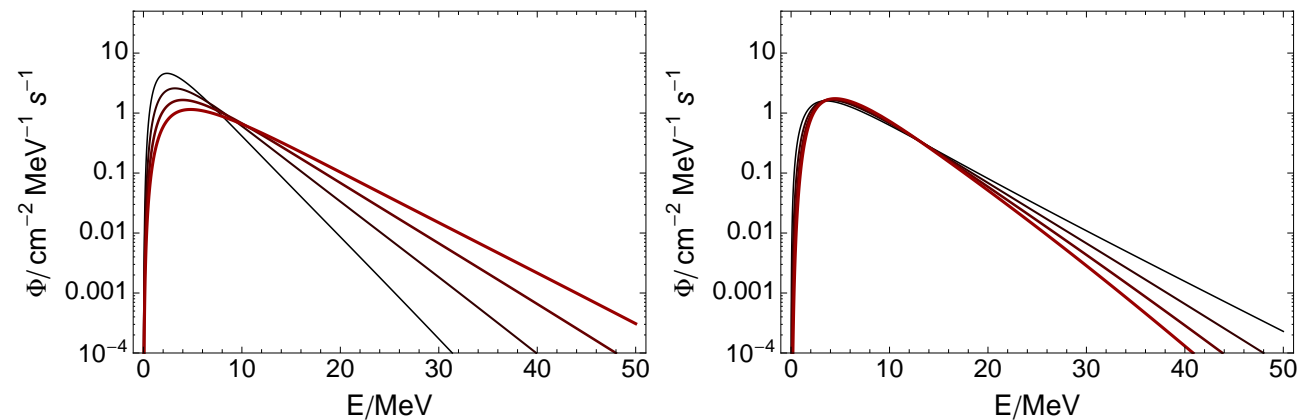

Figure 10: Examples of unoscillated flux, $\Phi_{w}^{0}(w=e, \bar{e}, x)$ (Eq. (15)), for different spectral parameters $E_{0 w}, \alpha_{w}$. Left: the curves of increasing thickness (increasing color intensity) correspond to $E_{0 w}=9,12,15,18 \mathrm{MeV}$, with $\alpha_{w}=3$. Right: the curves of increasing thickness (increasing color intensitiy) correspond to $\alpha_{w}=2,3,4,5$ with $E_{0 w}=15 \mathrm{MeV}$.

Fig. 10 shows $\Phi_{w}^{0}(w=e, \bar{e}, x)$ for different values of the the parameters $E_{0 w}, \alpha_{w}$ of the original flux $F_{w}^{0}$. It appears that $\Phi_{w}^{0}$ has a peak value of $\sim 1-5$ $\mathrm{cm}^{-2} \mathrm{~s}^{-1} \mathrm{MeV}^{-1}$ at $4-7 \mathrm{MeV}$, with an exponential decay at higher energy. As expected from Eq. (18), the decay is faster for smaller $\epsilon_{w}$, corresponding to 
lower average energy $E_{0 w}$ and/or larger $\alpha_{w}$. For $\alpha_{w}=3$, varying $E_{0 w}$ between 9 and $18 \mathrm{MeV}$ corresponds to a variation of the diffuse flux at $20 \mathrm{MeV}$ by about one order of magnitude, while variations are more modest (a factor of 2 or so) when varying $\alpha_{w}$ between 2 and 5 with $E_{0 w}$ fixed. The dependence on the parameters becomes stronger with increasing neutrino energy.
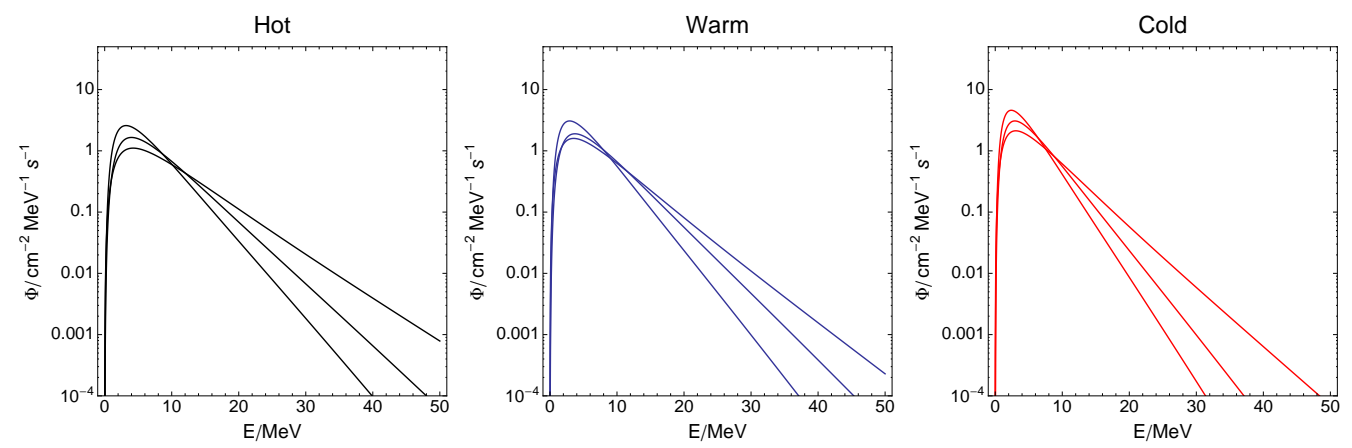

Figure 11: The fluxes $\Phi_{e}^{0}, \Phi_{\bar{e}}^{0}, \Phi_{x}^{0}$, defined in Eq. (15), for the three spectra examples in Table 1. For each of them, the colder to hotter spectra refer to $\nu_{e}, \bar{\nu}_{e}, \bar{\nu}_{x}$.

For further illustration, the fluxes $\Phi_{e}^{0}, \Phi_{\bar{e}}^{0}, \Phi_{x}^{0}$ for the H, W and C spectra (Table 1) are presented in fig. 11. Notice that the $\nu_{x}$ flux can easily be one order of magnitude larger than the $\nu_{e}$ one at $30-40 \mathrm{MeV}$. This already gives an idea of how flavor conversion can strongly enhance the potential of detection of the electron flavor components above realistic thresholds (sec. 3.5.4).
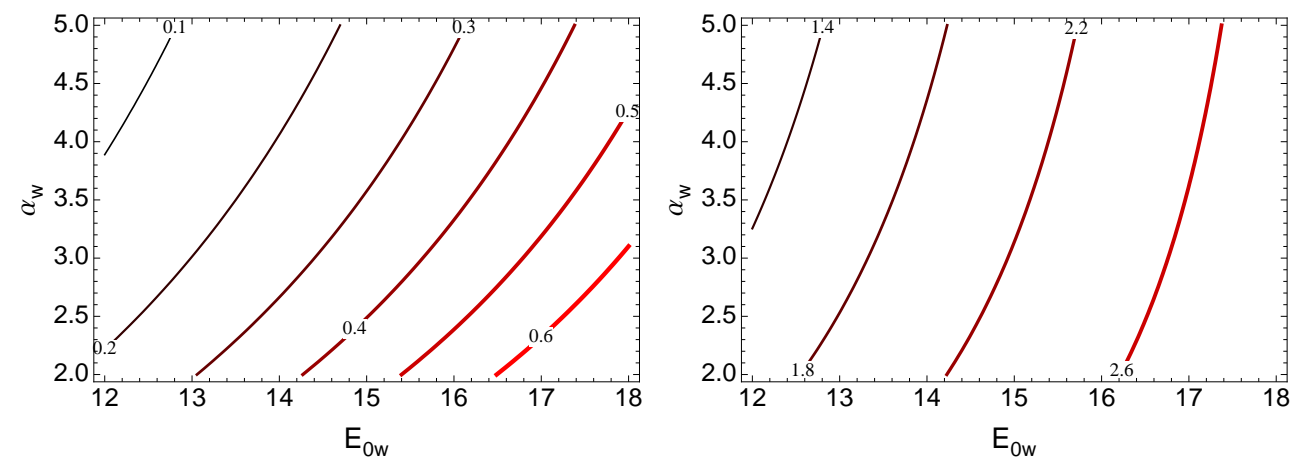

Figure 12: Isocontours of the total (unoscillated) flux, $\phi_{w}^{0}$, above the threshold energy $E_{t h}$, in $\mathrm{cm}^{-2} \mathrm{~s}^{-1}$, in the $E_{0 w}, \alpha_{w}$ plane. Left: $E_{t h}=19.3 \mathrm{MeV}$. Right: $E_{t h}=11.3 \mathrm{MeV}$.

The characteristics of the integrated flux $\phi_{w}^{0}$ reflect those already noted 
for the energy spectrum: for $E_{t h} \gtrsim 10 \mathrm{MeV}, \phi_{w}^{0}$ increases with the increasing average energy $E_{0 w}$ and with decreasing values of $\alpha_{w}$. Fig. 12 show this for $E_{t h}=19.3,11.3 \mathrm{MeV}$, that are both relevant for water Cherenkov detectors (sec. 4). In the figure one notes the dramatic increase (up to one order of magnitude!) of the flux as the energy cut is lowered, thus approaching the peak of the spectrum.

\begin{tabular}{|c|c|c|c|}
\hline \hline & \multicolumn{3}{|c|}{$\phi_{e}^{0}, \phi_{\bar{e}}^{0}, \phi_{x}^{0}$} \\
\hline & Hot & Warm & Cold \\
\hline \hline total & $17.2,13.8,11.5$ & $18.6,14.8,13.7$ & $22.4,18.6,15.7$ \\
\hline$E>11.3 \mathrm{MeV}$ & $1.44,2.22,2.90$ & $1.17,1.97,2.37$ & $0.64,1.17,1.91$ \\
\hline$E>19.3 \mathrm{MeV}$ & $0.14,0.35,0.74$ & $0.09,0.27,0.46$ & $0.03,0.09,0.30$ \\
\hline \hline
\end{tabular}

Table 3: The unoscillated fluxes at Earth integrated above energy thresholds of interest, in $\mathrm{cm}^{-2} \mathrm{~s}^{-1}$, for the three cases in Table 1 .

This is further illustrated in Table 3 , where the values of $\phi_{e}^{0}, \phi_{\bar{e}}^{0}, \phi_{x}^{0}$ are given for the $\mathrm{H}, \mathrm{W}$ and $\mathrm{C}$ spectra. The Table evidences the large differences between the fluxes in the different flavors and in the different cases. Among the total fluxes, integrated over all energies, the $\nu_{e}$ flux is larger: indeed, it has the same total energy than the other flavors, but each $\nu_{e}$ carries on average a lower energy. When the integration is restricted to increasingly high energy, the $\nu_{x}$ unoscillated flux starts to dominate, being higher than the $\nu_{e}$ flux by a factor of 3-10. For the $\mathrm{H}$ example, which has the most energetic $\nu_{x}, \phi_{x}^{0}$ is close (within a factor $\sim 3-4$ ) to the current SK limit, Eq. (2), thus confirming that experiments are already probing the interesting region of the parameters. For example, with a neutrino spectrum equally or more energetic than the $\mathrm{H}$ one, one can constrain the SNR normalization $[45]$.

\subsubsection{Dependence on the core collapse rate}

It is interesting to study what fraction of the DSNB is due to sources at cosmological distances, i.e., $z \gtrsim 1$. Fig. 13 addresses this question, showing the contributions to the flux of sources in bins of the form $[z, z+1]$, for the unoscillated $\bar{\nu}_{e}$ flux obtained with the $\mathrm{H}$ spectrum and the SNR in Eq. (5). It appears that the flux above $\sim 20 \mathrm{MeV}$ is practically all due to supernovae at $z<1$, while the contribution of more distant sources becomes increasingly 


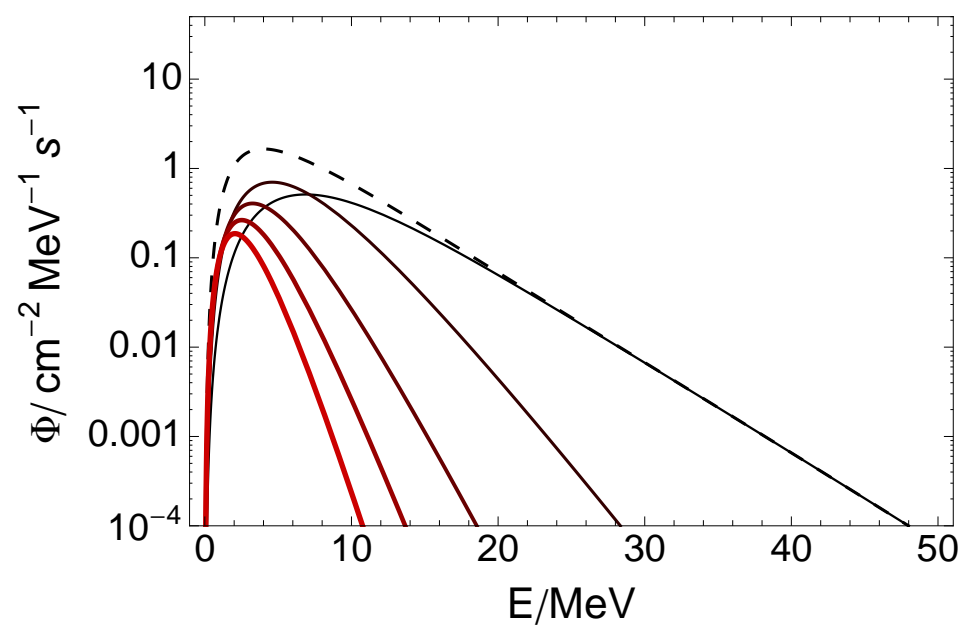

Figure 13: The contribution to the unoscillated $\bar{\nu}_{e}$ flux of sources in bins of increasing redshift, for the best fit SNR parameter $\beta=3.28$ [61]. The solid curves from thinner to thicker (darker to lighter color) refer to the intervals: $z=0-1, z=1-2, z=2-3$, $z=3-4$ and $z=4-5$. The dashed line is the total flux integrated over all redshifts. The parameters of the $\mathrm{H}$ case were used (Table 1 ).

important at decreasing energy: sources at $z \sim 2$ should be included to reproduce the flux at $10 \mathrm{MeV}$, and at $2 \mathrm{MeV}$ the dominant contribution is from sources at $z>2$. This feature is explained with the larger redshift of the energy of neutrinos emitted at larger distances. If we consider the total flux of neutrinos of all energies, the contributions of the first three redshift bins are about $\sim 40 \%, 35 \%, 14 \%$, while the remaining bins contribute for less than $\sim 10 \%$. See $[19]$ for further details.

How does the DSNB depend on the SNR parameters, $\beta, R_{S N}(0)^{8}$ ? This question is especially relevant if one considers the region allowed by supernova observations only (fig. 5), which is wider than that allowed by measurements of the star formation rate and exhibits a correlation between the normalization, $R_{S N}(0)$ and the power $\beta$. Fig. 14 shows the unoscillated $\bar{\nu}_{e}$ flux obtained with the $\mathrm{H}$ spectrum and with a set of point in the space $\left(\beta, R_{S N}(0)\right)$ that roughly map the $95.4 \%$ C.L. region in fig. 5 (see caption of fig. 14 for details). One can see that larger $\beta$ (faster growth of the SNR with $z$ ) corresponds to less energetic spectrum, reflecting the increased contribution to the flux of

\footnotetext{
${ }^{8}$ The dependence on $\alpha$ and $\gamma$ in Eq. (4) is weak, and negligible in first approximation.
} 


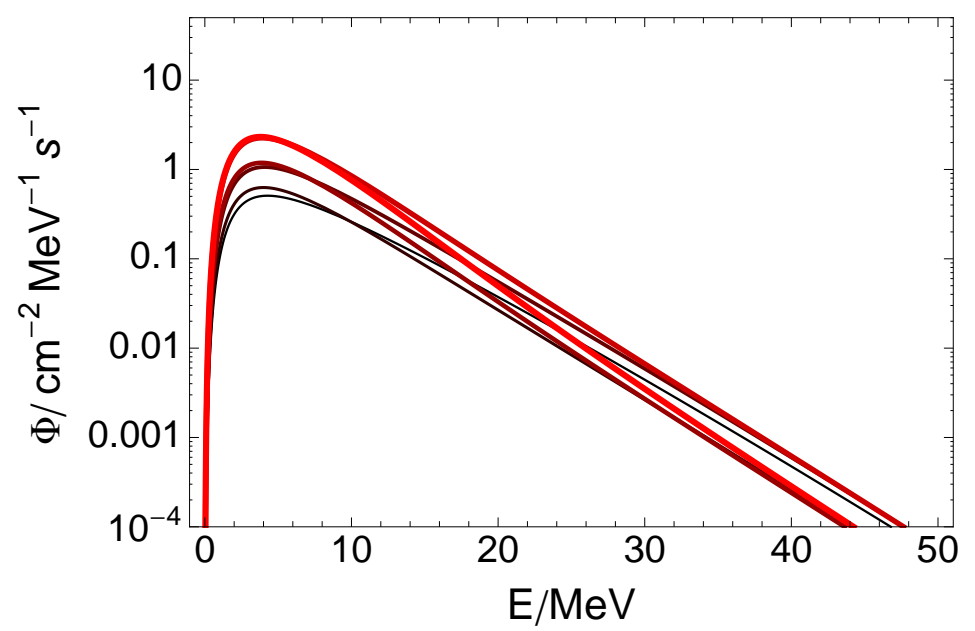

Figure 14: The unoscillated $\bar{\nu}_{e}$ flux calculated for different points in the largest allowed region of the parameters $\beta, R_{S N}(0)$ (fig. 5, outer dashed contour). The curves from thinner to thicker (darker to lighter color) refer to the points: $\left(\beta, R_{S N}(0)\right)=$ $(1.6,0.9),(3.2,0.4),(4.6,0.3),(2.6,1),(5.6,0.3),(4,0.85)$, where $R_{S N}(0)$ is in units of $10^{-4} \mathrm{yr}^{-1} \mathrm{Mpc}^{-3}$. The parameters of the $\mathrm{H}$ spectrum were used (Table 1 ).

the more redshifted neutrinos from cosmological sources. At certain points of the energy spectrum, the flux can vary by up to a factor of 5 as an effect of the variation of the SNR parameters, however the variation does not exceed a factor of 2 for the integrated fluxes above thresholds on interest.

\subsubsection{The $\bar{\nu}_{e}$ and $\nu_{e}$ fluxes in a detector}

When oscillation effects are included, the $\bar{\nu}_{e}$ and $\nu_{e}$ components of the DSNB receive a contribution from the original $\nu_{x}$ flux produced inside the star, eq. (15). The oscillated $\nu_{e}$ and $\bar{\nu}_{e}$ fluxes are described in figs. 15 and 16 and in Tables 4 and 5 for the $\mathrm{H}, \mathrm{W}$ and $\mathrm{C}$ spectra, as well as for the flux that best fits the SN1987A data (Eq. (14)). All results refer to the extreme values of the survival probabilities $p, \bar{p}$, as they illustrate the maximum range of variation of the DSNB with the varying oscillations parameters. Intermediate cases can be calculated from the unoscillated fluxes in the different flavors (sec. 3.5.2, Table 3).

From the energy spectra in fig. 15 one can see that large variations (up to a factor of 3 or so) in the $\bar{\nu}_{e}$ flux can be expected for each model above 19.3 $\mathrm{MeV}$ as a consequence of the wide range of variation of $\bar{p}$. The $\nu_{e}$ flux instead is always within $30-40 \%$ from the unoscillated flux $\Phi_{x}^{0}$ as a consequence of 

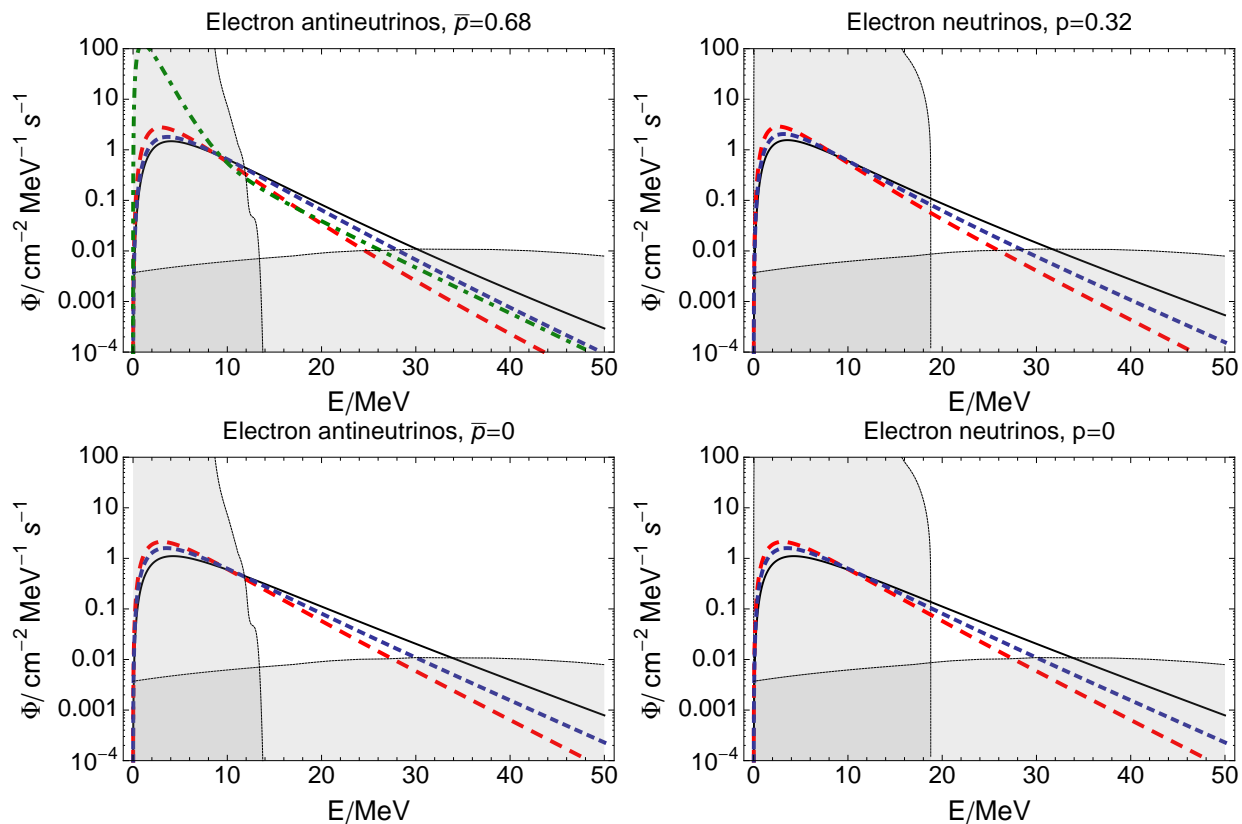

Figure 15: Examples of energy spectra of the $\nu_{e}$ and $\bar{\nu}_{e}$ components of the DSNB from the literature for the two extreme values of $p$ and $\bar{p}$. The solid, short dashed and long dashed curves are for the Hot (H), Warm (W) and Cold (C) spectra (Table 1). The dashed-dotted line corresponds to the best fit of a multi-parameter statistical analysis of SN1987A data [21] (Eq. 14). All the curves were obtained for the piecewise SNR function (Eq. 5) with $R_{-4}=1$. The four panels also show backgrounds due to other neutrino sources (see sec. 4.1): $\bar{\nu}_{e}$ s from the atmosphere [130] and from reactors (below $\sim 11 \mathrm{MeV}$, inclusive of oscillation effects, from [58]), and $\nu_{e} \mathrm{~s}$ from the atmosphere [130] and from the Sun (below $\sim 19 \mathrm{MeV}$ ) [131]. All these background fluxes are for the Kamioka site. 
the small component due to $\Phi_{e}^{0}, p \leq 0.32$. Thanks to the larger contribution of the $\nu_{x}$ original flux, the $\nu_{e}$ spectrum is always more energetic than the $\bar{\nu}_{e}$ one. The $\nu_{e}$ and $\bar{\nu}_{e}$ diffuse fluxes can be equal in the limiting case $p=\bar{p}=0$. This is realized for inverted (normal) hierarchy if collective oscillations are maximally effective for $\bar{\nu}_{e}\left(\nu_{e}\right)$ and completely suppressed for $\nu_{e}\left(\bar{\nu}_{e}\right)$ (see Table 2).

Large variations in the flux are also seen for fixed $p, \bar{p}$ and varying spectral model, as already commented about the unoscillated fluxes. Expectedly, at high energy the $\mathrm{H}$ spectrum gives a flux that is much larger (up to one order of magnitude at $50 \mathrm{MeV}$ ) that the other spectra. For the SN1987A best fit spectrum the DSNB has a high peak at $2 \mathrm{MeV}$, reflecting the very high $\bar{\nu}_{e}$ original flux, and exceeding the other predictions by two orders of magnitude. For the energies of interest here, however, the flux is intermediate between the $\mathrm{W}$ and $\mathrm{C}$ spectra cases.

\begin{tabular}{|c|c|c|c|c|}
\hline \hline & Hot & Warm & Cold & SN1987A best fit (Eq. (14)) \\
\hline \hline total & 13.2 & 14.6 & 18.1 & 305 \\
\hline$E>11.3 \mathrm{MeV}$ & 2.43 & 2.10 & 1.41 & 1.3 \\
\hline$E>19.3 \mathrm{MeV}$ & 0.47 & 0.33 & 0.16 & 0.21 \\
\hline \hline
\end{tabular}

Table 4: Integrated $\bar{\nu}_{e}$ flux above thresholds of interest, in $\mathrm{cm}^{-2} \mathrm{~s}^{-1}$, for $\bar{p}=0.68$. All parameters are as in fig. 15. The results for the SN1987A best fit flux are from [21], and are in agreement with those of [36], where a different method of analysis is used.

\begin{tabular}{|c|c|c|c|}
\hline \hline & Hot & Warm & Cold \\
\hline \hline total & 13.31 & 15.3 & 17.8 \\
\hline$E>11.3 \mathrm{MeV}$ & 2.43 & 1.98 & 1.51 \\
\hline$E>19.3 \mathrm{MeV}$ & 0.55 & 0.35 & 0.21 \\
\hline \hline
\end{tabular}

Table 5: Integrated $\nu_{e}$ flux above thresholds of interest, in $\mathrm{cm}^{-2} \mathrm{~s}^{-1}$, for $p=0.32$. All parameters are as in fig. 15 .

The integrated fluxes are given in fig. 16 and in Tables 4 and 5 for completeness. They reflect the features already noted for the unoscillated fluxes: the fast (exponential) decay of the flux with the increase of threshold energy $E_{t h}$, and the variation by a factor of $\sim 2$ of the flux at high energy 

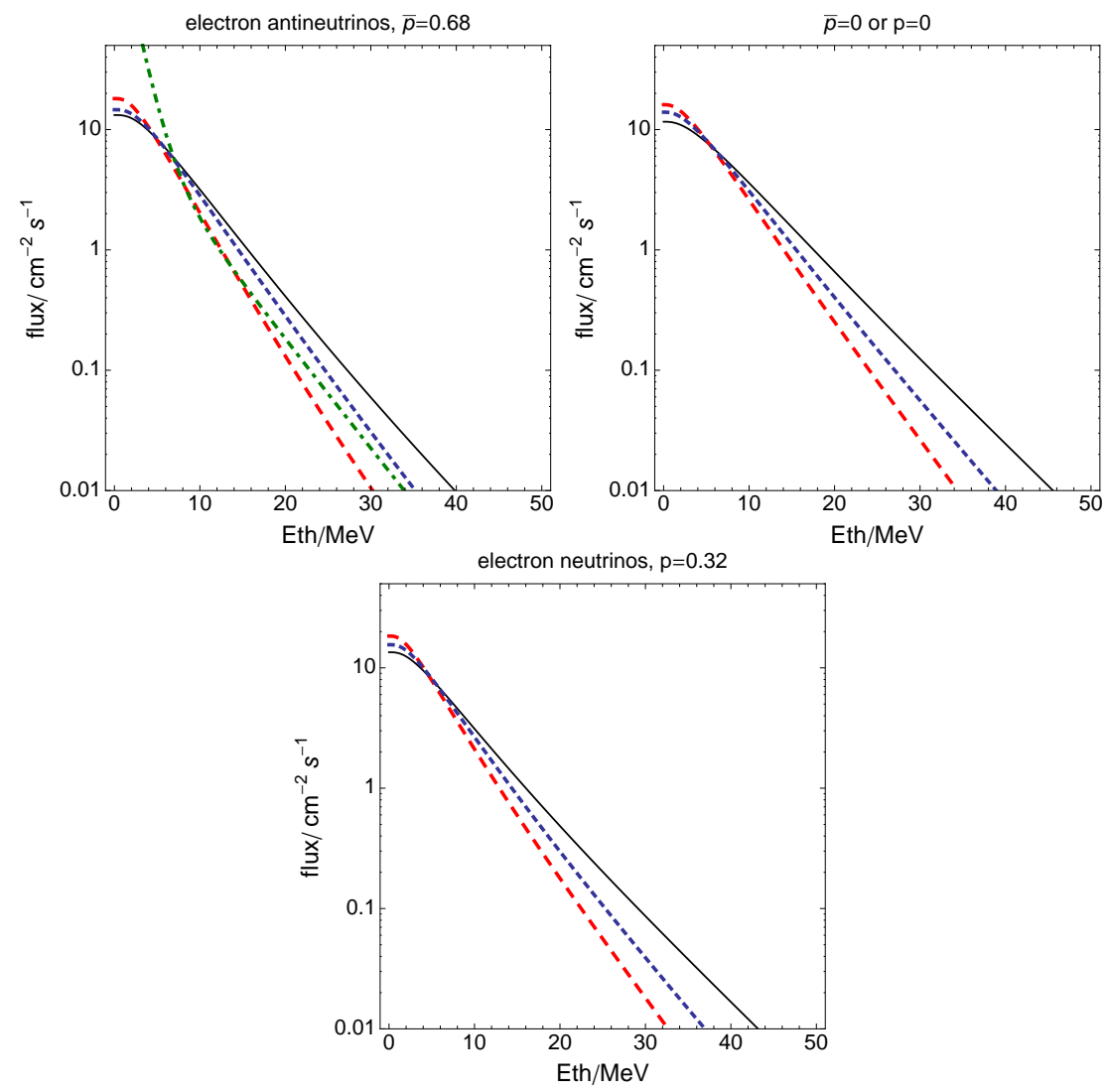

Figure 16: Diffuse $\nu_{e}$ and $\bar{\nu}_{e}$ fluxes integrated above a threshold energy $E_{t h}$, as a function of $E_{t h}$ for selected values of $p, \bar{p}$. The middle panel refers to the case in which the $\bar{\nu}_{e}$ and $\nu_{e}$ fluxes both undergo complete flavor permutation into $\nu_{x}$, and therefore are equal. The solid, short dashed and long dashed curves are for the Hot $(\mathrm{H})$, Warm $(\mathrm{W})$ and Cold $(\mathrm{C})$ spectra (Table 1). The dashed-dotted line corresponds to the best fit of a multi-parameter statistical analysis of SN1987A data [21] (Eq. (14)). All other parameters are as in fig. 15. 
depending on the spectrum adopted. Again, the $\nu_{e}$ flux is generally larger above realistic thresholds due to the larger flavor permutation.

\subsubsection{Diffuse flux from failed supernovae}

Analogously to what done so far for neutrinos from neutron star-forming collapses, one can calculate the diffuse flux of neutrinos from black holeforming ones, $\Phi_{B H}[42,43,92,44,33,31]$, using the spectra in fig. 6 and considering that neutron star-forming (black hole-forming) collapses amount to a fraction $f_{N S}=0.78-0.91\left(1-f_{N S}=0.09-0.22\right)$ of the total (sec. 3.2).
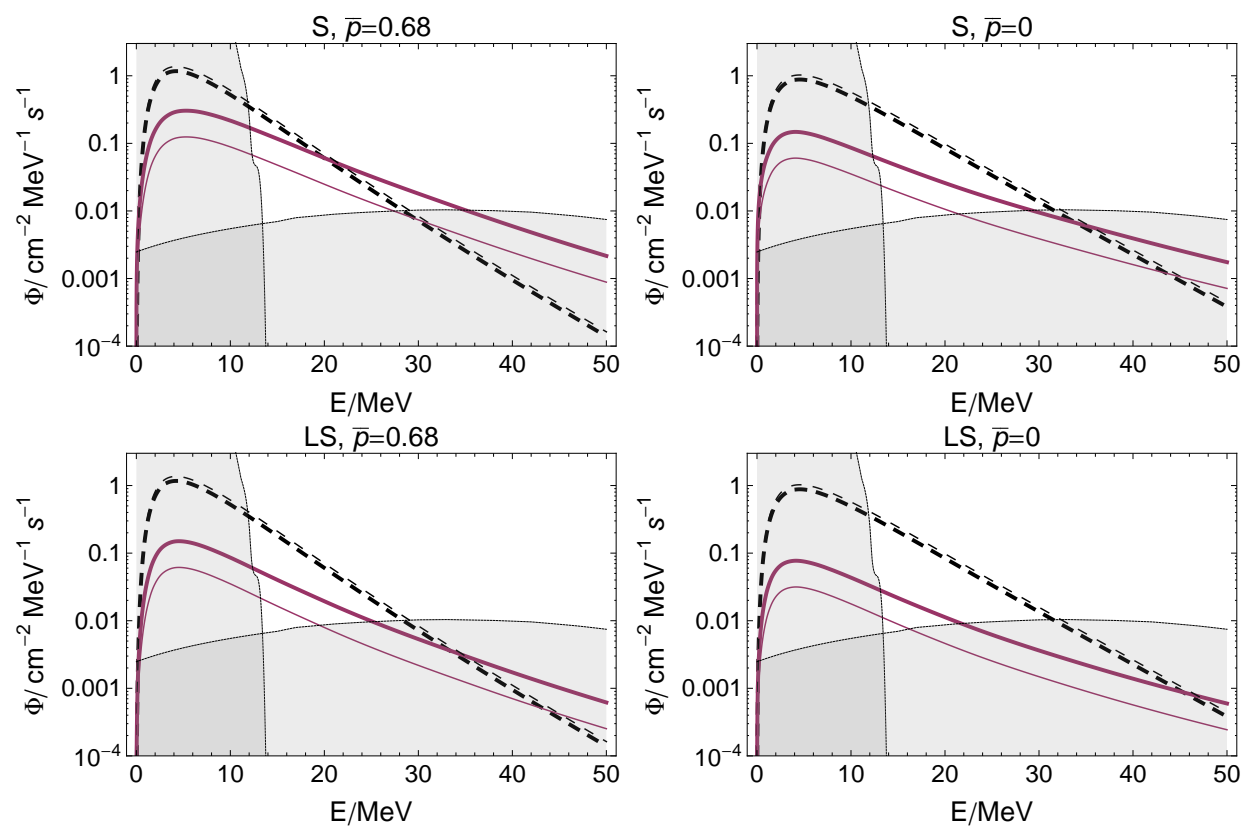

Figure 17: From [42, 92]: the diffuse flux of $\bar{\nu}_{e}$ at Earth from direct black hole-forming collapses, i.e. failed supernovae (solid lines), for the Shen at al. (S) and Lattimer-Swesty (LS) equation of state and different values of the survival probability $\bar{p}$. The flux from neutron star-forming collapses is also plotted (dashed curves). Direct black hole -forming collapses are assumed to be $22 \%$ (thick curves) or $9 \%$ (thin curves) of the total. The $\bar{\nu}_{e}$ atmospheric and reactor backgrounds are shown for comparison.

Results are given in fig. 17 for the $\bar{\nu}_{e}$ component of $\Phi_{B H}$ (similar conclusions hold for the $\nu_{e}$ component, see e.g., [92]) and the extreme values of $f_{N S}$ and of $\bar{p}$, which is assumed to be the same for both types of collapses. For comparison, examples of diffuse flux from neutron star-forming collapses are shown; they were calculated using the parameters $E_{0 \bar{e}}=15 \mathrm{MeV}, E_{0 x}=18$ 
$\mathrm{MeV}, L_{\bar{e}}=L_{x}=5 \cdot 10^{52} \mathrm{ergs}, \alpha_{\bar{e}}=3.5$ and $\alpha_{x}=2.5$, that is similar to the $\mathrm{H}$ spectrum case. As expected, $\Phi_{B H}$ has hotter spectrum compared to the flux from neutron star-forming collapses, and thus it is increasingly important at higher energy. Oppositely to the case of neutrinos from neutron star-forming collapses, $\Phi_{B H}$ is larger for minimal permutation $(\bar{p}=0.68)$ [87], because of the especially luminous original fluxes in the electron flavor (fig. 6). The dependence of the original fluxes on the EoS is evident in $\Phi_{B H}$.

Fig. 17 evidences that $\Phi_{B H}$ might dominate already at $E \sim 22 \mathrm{MeV}$, implying a strong effect at SuperKamiokande. For the most favorable parameters the total flux from both types of collapses above $19.3 \mathrm{MeV}$ is more than twice as large as the case of $100 \%$ neutron star-forming collapses. It reaches the value $\phi_{B H} \simeq 0.89 \mathrm{~cm}^{-2} \mathrm{~s}^{-1}$, tantalizingly close to the current upper limit. It is more likely, however, that $\Phi_{B H}$ becomes dominant only above $30-40 \mathrm{MeV}$, as the figure shows. If so, its effect would be below the sensitivity of SK - which would therefore place limits on neutrinos from failed supernovae [43] - but might be visible at the more massive and more sensitive detectors of the next generation.

\section{Detection: the diffuse neutrino flux at neutrino observatories}

\subsection{The energy window}

A fortunate circumstance makes the DSNB detectable: the fact that part of it falls in a relatively quiet region of the neutrino spectrum, and precisely above the typical energies of neutrinos from nuclear processes $\left(E_{n u c} \lesssim 18\right.$ $\mathrm{MeV}$ ) and below the bulk of the flux of neutrinos from cosmic rays (atmospheric neutrinos, $E_{\text {atm }} \gtrsim 10^{2} \mathrm{MeV}$ ). These neutrino fluxes are for the most part ineliminable backgrounds, and thus place a natural limit to the sensitivity of any experiment to the DSNB. Here I discuss the generalities of the $\nu_{e}$ and $\bar{\nu}_{e}$ components of these backgrounds, which dominate the relevant detection channels. They are shown in fig. 18. Detector-specific backgrounds are discussed in secs. 4.4-4.6.

- solar neutrinos. Electron, muon and tau neutrinos from the sun (with $\nu_{\mu}, \nu_{\tau}$ generated by oscillations of originally produced $\nu_{e} \mathrm{~s}$ ) extend to $\sim$ $16 \mathrm{MeV}$ energy if they come from the ${ }^{8} B$ chain, and even to $\sim 19 \mathrm{MeV}$ if produced through the hep process. Reaching $\sim 10^{2} \mathrm{~cm}^{-2} \mathrm{~s}^{-1} \mathrm{MeV}^{-1}$, the hep flux dominates over the DSNB in the whole energy range nearly up to its endpoint. Its $\nu_{e}$ component is shown in fig. 18, where the 


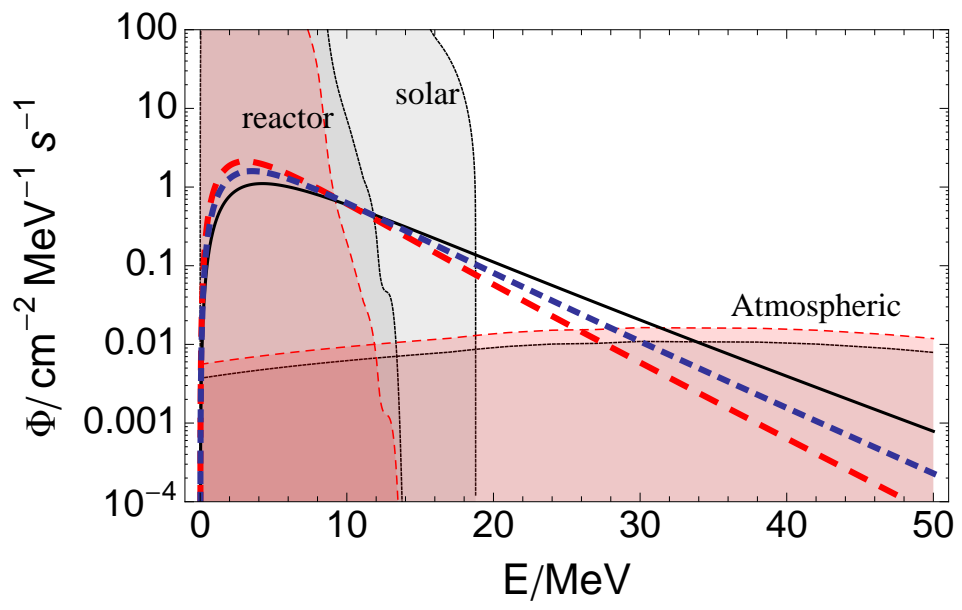

Figure 18: Backgrounds fluxes: $\bar{\nu}_{e} \mathrm{~s}$ from reactors (taken from [58]) and from the atmosphere [130], and $\nu_{e}$ from the Sun [131] and from the atmosphere [130]. These backgrounds are compared with the signal from the DSNB with $p=\bar{p}=0$ for different neutrino spectra, as in fig. 15. The atmospheric and reactor fluxes are shown for the Kamioka (solid, gray) and Homestake (dashed, red) sites. The atmospheric fluxes of $\nu_{e}$ and $\bar{\nu}_{e}$ are very similar, so only one of them is plotted. The calculations of the background fluxes include oscillation effects, which are responsible for the visible modulation of the reactor spectrum.

\begin{tabular}{|c|c|}
\hline \hline Detector location & energy window $(\mathrm{MeV})$ \\
\hline \hline Kamioka (J) & $11.1-28.1$ \\
Frejus (F) & $10.8-26.4$ \\
Kimballton (US) & $10.6-28.1$ \\
Pyhasalmi (FIN) & $9.7-25.1$ \\
Pylos (GR) & $9.4-28.1$ \\
Homestake (US) & $9.0-26.4$ \\
Henderson (US) & $8.9-27.2$ \\
Hawaii (US) & $8.4-29.0$ \\
Wellington (NZ) & $8.2-27.2$ \\
\hline \hline
\end{tabular}

Table 6: The location-dependent energy window for the detection of the $\bar{\nu}_{e}$ component of the DSNB. This window is defined (optimistically) as the interval where the signal exceeds the background fluxes of atmospheric and reactor neutrinos. The table is adapted from [58], where the parameters used for the DSNB are $R_{-4}=0.87, E_{0 \bar{e}}=15.4 \mathrm{MeV}$, $E_{0 x}=15.7 \mathrm{MeV}, \alpha_{\bar{e}}=4.2, \alpha_{x}=2.5$ and $\bar{p}=0.71$. 
effect of oscillations in the Sun has been included using the best fit oscillation parameters in Eq. (8).

- reactor antineutrinos. Nuclear powerplants are a copious source of electron antineutrinos of energies up to $\sim 14 \mathrm{MeV}$. This reactor flux acquires a muon and tau component on the way to a detector due to oscillations [132]. Very important as a signal for oscillation tests, the reactor antineutrino flux is a serious obstacle to the study of diffuse antineutrinos from supernovae. For example, at the Kamioka site the reactor flux is as high as $\sim 10^{2} \mathrm{~cm}^{-2} \mathrm{~s}^{-1} \mathrm{MeV}^{-1}$ at $8 \mathrm{MeV}$, and dominates over the DSNB below $\sim 12 \mathrm{MeV}$ (fig. 18). The lower reactor flux in less nuclearized areas would allow a slightly larger energy window for the DSNB, down to $9 \mathrm{MeV}$ for Homestake (where the reactor flux is lower by a factor of $\sim 30$ compared to Kamioka) and even to 8.2 $\mathrm{MeV}$ for a detector in New Zealand (table 6). Even in the best case, however, the reactor flux obscures the peak of the DSNB.

- geoneutrinos. Even in the ideal scenario of complete absence of reactors, there is still another low energy background: a flux of $\bar{\nu}_{e}$ from the natural radioactivity of the Earth. These geoneutrinos have been seen by KamLAND [133] and Borexino [134], and - according to calculations [5] - they should dominate over the DSNB below 3.26 MeV, where the spectrum of $\bar{\nu}_{e}$ from ${ }^{214} B i$ ends. This energy is sufficiently low to expose the peak of the DSNB at 5-7 MeV; however the possibility to reach such lower threshold is highly speculative, and is presented here only as motivation for minimizing the reactor flux.

- atmospheric neutrinos. Collisions of cosmic rays with the Earth's atmosphere generate a shower of $\nu_{e}, \bar{\nu}_{e}, \nu_{\mu}, \bar{\nu}_{\mu}$ (and $\nu_{\tau}, \bar{\nu}_{\tau}$ via oscillations) that appears in neutrino detectors primarily in the energy window $0.1-10^{3}$ $\mathrm{GeV}$. Still, a low energy tail of this atmospheric flux extends down to few $\mathrm{MeV}$. The spectrum of $\nu_{e} \mathrm{~s}$ and $\bar{\nu}_{e} \mathrm{~s}$ in this tail [130] is shown in fig. 18. Oscillation effects have not been taken into account, since they are at the level of $5-10 \%$ at these energies [135]. The figure shows that typically the atmospheric flux exceeds the DSNB above $\sim 30 \mathrm{MeV}$ or so depending on the intensity and spectrum of the DSNB. It should also be noticed that the atmospheric neutrino flux is location-dependent due to the dependence of the cosmic ray flux on the latitude, and increases as one moves towards the magnetic poles of our planet. For example, 
the atmospheric flux is about $\sim 1.5$ times higher at Homestake than at Kamioka [58], resulting in a smaller energy window for the DSNB. This appears in fig. 18 and in Table 6 [58], which gives the energy windows for $\bar{\nu}_{e}$ detection in different locations.

It is interesting to consider whether the fluxes discussed here are truly ineliminable backgrounds or can in principle be distinguished from the DSNB. While the answer ultimately depends on technology, here I observe that only the atmospheric neutrino flux is truly similar to the DSNB: it shares the same energy window, has similar flavor composition, and has the same isotropic distribution in direction. The other fluxes are not isotropic, and therefore can be distinguished in principle, while most likely not in practice.

Besides the neutrino fluxes discussed here, one should consider a number of non-neutrino processes and various instrumental effects that also constitute background for a given detector and that generally restrict the energy window compared to what discussed here. These backgrounds are described in sec. 4.2 .

\subsection{Detection concepts}

Neutrinos in a detector are studied through the products of their interaction with electrons and nuclei. For a charged current process of the type $\nu_{e}+{ }_{Z}^{A} X \rightarrow{ }_{Z+1}^{A} Y+e^{-}$, the rate of events with electrons of observed kinetic energy $E_{e}$ is

$$
\frac{d N_{e}}{d E_{e}}=N_{T} \int_{-\infty}^{+\infty} d E_{e}^{\prime} \mathcal{R}\left(E_{e}, E_{e}^{\prime}\right) \mathcal{E}\left(E_{e}^{\prime}\right) \int d E \Phi_{e}(E) \frac{d \sigma\left(E_{e}^{\prime}, E\right)}{d E_{e}^{\prime}}
$$

where $E_{e}^{\prime}$ is the true energy of the electron, $N_{T}$ is the number of target nuclei in the fiducial volume and $\mathcal{E}$ represents the detection efficiency. Here $d \sigma\left(E_{e}^{\prime}, E\right) / d E_{e}^{\prime}$ is the differential cross section of the detection reaction and $\mathcal{R}\left(E_{e}, E_{e}^{\prime}\right)$ is the energy resolution function. An expression analogous to (19) holds for the events due to the $\bar{\nu}_{e}$ flux, $\Phi_{\bar{e}}$.

From what discussed so far, it appears that the detection of diffuse supernova neutrinos requires a detector with: (i) large mass (at least comparable with SK, 50 kt), (ii) high detection cross section (iii) good energy resolution (to identify and study the DSNB energy window) and (iv) excellent discrimination of the signal over the background. Currently neutrino observatories are entering their mature phase, and several technologies are now available 


\begin{tabular}{|c|c|c|c|c|c|}
\hline Concept & $\begin{array}{l}\text { energy } \\
\text { window } \\
(\mathrm{MeV})\end{array}$ & $\begin{array}{l}\text { detection } \\
\text { processes }\end{array}$ & experiment & $\begin{array}{l}\text { fiducial } \\
\text { mass (kt) }\end{array}$ & $\begin{array}{l}\text { events } \\
\text { per year }\end{array}$ \\
\hline \multirow[t]{3}{*}{$\overline{\mathrm{H}} \mathrm{H}_{2} \mathrm{O}$} & \multirow{3}{*}{$\begin{array}{c}19.3-30 \\
{[17.3-30]}\end{array}$} & \multirow{3}{*}{$\begin{array}{c}\bar{\nu}_{\mathbf{e}}(\mathbf{p}, \mathbf{n}) \mathbf{e}^{+} \\
\bar{\nu}_{e}\left({ }^{16} O, X\right) e^{+} \\
\nu_{w}\left(e^{-}, e^{-}\right) \nu_{w} \\
\nu_{w}(p, p) \nu_{w} \\
\nu_{w}\left({ }^{16} O, X\right) \nu_{w} \\
\end{array}$} & SK [15] & 22.5 & $0.23-1.0$ \\
\hline & & & Hyper-K [48] & 560 & $5.7-25.3$ \\
\hline & & & & & \\
\hline $\mathrm{H}_{2} \mathrm{O}+\mathrm{Gd}$ & $11.3-30$ & " same as $\mathrm{H}_{2} \mathrm{O}$ & SuperK-Gd [57] & 22.5 & $0.93-2.3$ \\
\hline \multirow[t]{3}{*}{ Scintillator } & \multirow[t]{3}{*}{$\sim 11-30$} & \multirow{3}{*}{$\begin{array}{c}\bar{\nu}_{\mathbf{e}}(\mathbf{p}, \mathbf{n}) \mathbf{e}^{+} \\
\nu_{e}\left({ }^{12} C, X\right) e^{-} \\
\bar{\nu}_{e}\left({ }^{12} C, X\right) e^{+} \\
\nu_{w}\left(e^{-}, e^{-}\right) \nu_{w} \\
\nu_{w}(p, p) \nu_{w} \\
\nu_{w}\left({ }^{12} C, X\right) \nu_{w} \\
\end{array}$} & JUNO [55] & 17 & $0.8-1.9$ \\
\hline & & & RENO-50 [56] & 18 & $0.8-2.0$ \\
\hline & & & & & \\
\hline Argon & $\bar{c} \sim 18-30$ & $\begin{array}{c}\nu_{\mathbf{e}}\left({ }^{40} \mathbf{A r}, \mathbf{X}\right) \mathbf{e}^{-} \\
\bar{\nu}_{e}\left({ }^{40} A r, X\right) e^{+} \\
\nu_{w}\left(e^{-}, e^{-}\right) \nu_{w} \\
\nu_{w}\left({ }^{40} A r, X\right) \nu_{w}\end{array}$ & DUNE [52] & up to 40 & up to 1.0 \\
\hline
\end{tabular}

Table 7: Summary of running (SK only) and near-future detectors for the DSNB having mass above $10 \mathrm{kt}$. The neutrino energy windows are indicative (as they depend on a number of factors, see e.g. sec. 4.4.2) and refer to the main detection process, which is highlighted in bold. For water Cherenkov I give the energy windows of both the first and second search of SK (in brackets, see sec. 4.4.2); the rates are for the more conservative window. Here $X$ stands for any final state and $\nu_{w}$ indicates a neutrino or antineutrino of any flavor. The intervals of event rates include the different example spectra $\mathrm{H}, \mathrm{W}$ and $\mathrm{C}$ (Table 1) and different oscillation scenarios. 
to achieve these requirements. They are illustrated in Table 7, and reviewed briefly in this section. A more detailed discussion on specific designs is given in secs. 4.4-4.5.

- Water and water-based detectors. Water Cherenkov detection is probably the oldest and best known technology. It has already been used successfully to detect supernova neutrinos in the SN1987A event [126, 127]. SK has proven the feasibility of this technology for tens of kilotons mass, and - thanks to its robustness and and contained cost - masses up to $1 \mathrm{Mt}$ are considered realistic (sec. 4.4).

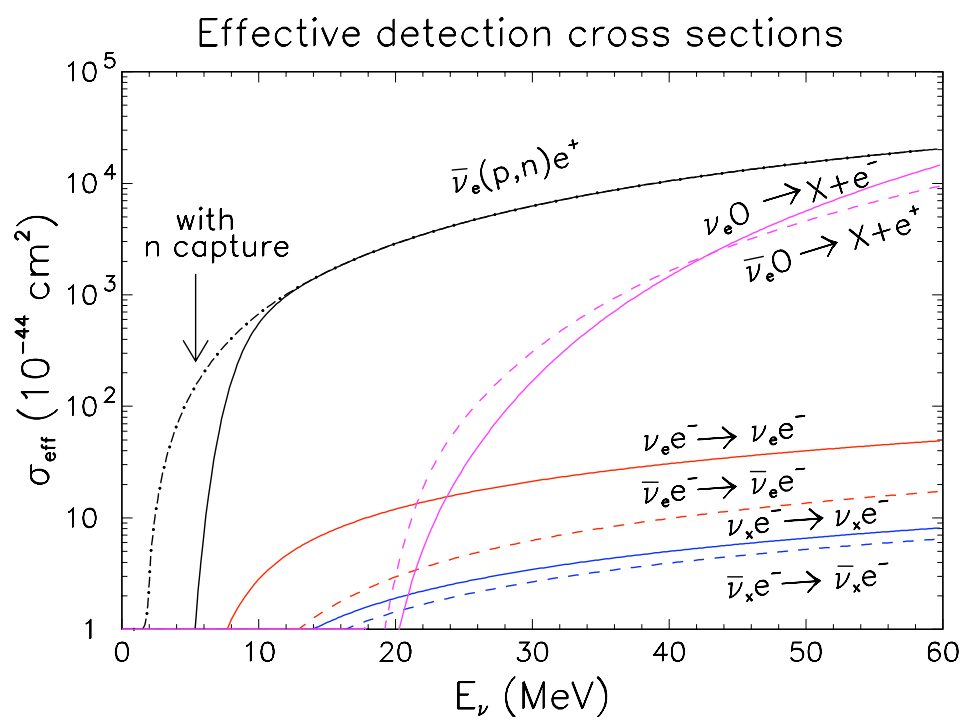

Figure 19: Effective cross sections for neutrinos in water [138], including the SK energy resolution and threshold effects. Figure from [18].

In water, supernova neutrinos undergo several scattering processes with the production of an electron or positron (energies are sub-threshold of muon and tau production) that can be detected through its Cherenkov cone. These processes (Table 7) are elastic scattering of neutrinos of all flavors on electrons and charged-current interactions of $\nu_{e}$ and $\bar{\nu}_{e}$ on hydrogen and oxygen nuclei. Their cross sections are shown in fig. 19. In realistic energy windows, inverse beta decay $\left(\bar{\nu}_{e}+p \rightarrow n+e^{+}\right)$ exceeds all other channels by at least one order of magnitude in cross section, and therefore it dominates a signal from the DSNB. All other channels can be neglected in first approximation. 
The energy resolution $\mathcal{R}\left(E_{e}, E_{e}^{\prime}\right)$ of a water Cherenkov detector depends on photocatode coverage. For example, the resolution of SK is well modeled by a Gaussian function of width $\Delta / \mathrm{MeV} \simeq(0.5-0.6) \times$ $\sqrt{E_{e} / \mathrm{MeV}}[136,18]$, corresponding to a resolution of $\sim 11-13 \%$ at 20 $\mathrm{MeV}$. At energies above 5-7 MeV, the (hardware) detection efficiency is very good, being close to $100 \%$ at SK [137].

The DSNB detection in water is background-dominated. Among backgrounds from other neutrino fluxes, events from solar neutrinos can be subtracted very effectively: they are due mostly to elastic scattering on electrons, which is directional (the emitted electron is nearly collinear with the incoming neutrino) and therefore pointing back to the Sun. The events from reactors and atmospheric neutrinos instead are mostly due to interactions with nuclei; they have little directional information, and thus are likely ineliminable backgrounds. Water detectors are also limited by other two backgrounds: events from spallation and events due to atmospheric invisible muons ${ }^{9}$ in the detector. At SK, most spallation events are excluded by a combination of an energy cut and of other techniques (see sec. 4.4.2), while invisible muons are included in data analyses (see sec. 4.3).

A relatively recent chapter in the development of water-based detectors is the idea to use of a Gadolinium compound dissolved in water for enhanced signal discrimination over background [57]. Gadolinium is a strong neutron capturer, already used in the past for the detection of reactor neutrinos (see e.g. [141]). The capture of a neutron on $\mathrm{Gd}$ is followed by gamma ray emission from de-excitation; the detection in coincidence of the gamma ray and positron from inverse beta decay allows to distinguish this process from spallation and from invisible muons in most cases ${ }^{10}$. This results in a strong reduction of backgrounds, by a factor of $\sim 5$ for invisible muons and by at least an order of magnitude for spallation $[57,18]$. Therefore, the energy window would extend down to the barrier posed by reactor neutrinos.

\footnotetext{
${ }^{9}$ The wording "invisible muon" denotes a muon whose only signature in the detector is the track of the electron (or positron) produced by its decay. See e.g. [129].

${ }^{10} \mathrm{~A}$ fraction of spallation and invisible muon events is accompanied by neutrons, and of these, some will be indistinguishable from inverse beta decay events in water with Gd. Still the reduction in background is expected to be substantial [137].
} 
Feasible at tens of kilotons scale, the water+Gadolinium technology might be impractical at larger masses. Still, it is a very attractive idea for a fast and cost-effective enhancement of current detectors (see sec. $4.5)$.

- Liquid scintillator. Another well known technology, liquid scintillator detection has been used extensively in studies of neutrinos of all types, including neutrinos from galactic supernovae [142, 143, 144]. Current liquid scintillator detectors reach $1 \mathrm{kt}$ mass, and a scaling of about one order of magnitude is envisioned [55, 56]. Made of organic materials of the type $C_{n} H_{2 n}$, scintillator can detect supernova neutrinos via elastic scattering on electrons and scattering on hydrogen and carbon nuclei (Table 4.2). Like in water, inverse beta decay dominates the

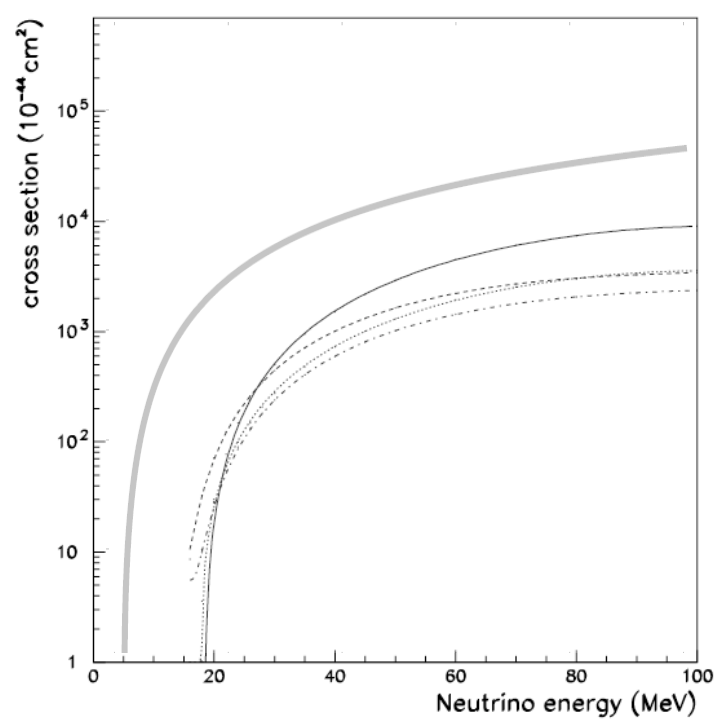

Figure 20: Cross sections for neutrino scattering on nuclei relevant to a liquid scintillator experiment: inverse beta decay (thick solid), $\nu_{e}$ and $\bar{\nu}_{e}$ charged current scattering on ${ }^{12} \mathrm{C}$ (thin solid and dashed), $\nu_{e}$ and $\bar{\nu}_{e}$ neutral current scattering on ${ }^{12} \mathrm{C}$ (dotted and dot-dashed). Figure adapted from [145].

event rates. Compared to water, scintillator offers better background discrimination and better energy resolution. Spallation and invisible 
muons ${ }^{11}$ are distinguished, for the most part, from inverse beta decay because the latter is accompanied by a gamma ray from the neutron capture on a free proton, $n(p, d) \gamma$. The coincident positron can be detected with energy resolution up to one order of magnitude better than water: $\Delta / \mathrm{MeV}=0.03 \sqrt{E_{e} / \mathrm{MeV}}$, i.e., less than $1 \%$ at $20 \mathrm{MeV}$ [55]. The same scaling to Mt mass as water detectors does not appear realistic, however. Thus, scintillator detectors might be ultimately limited by low statistics (sec. 4.5).

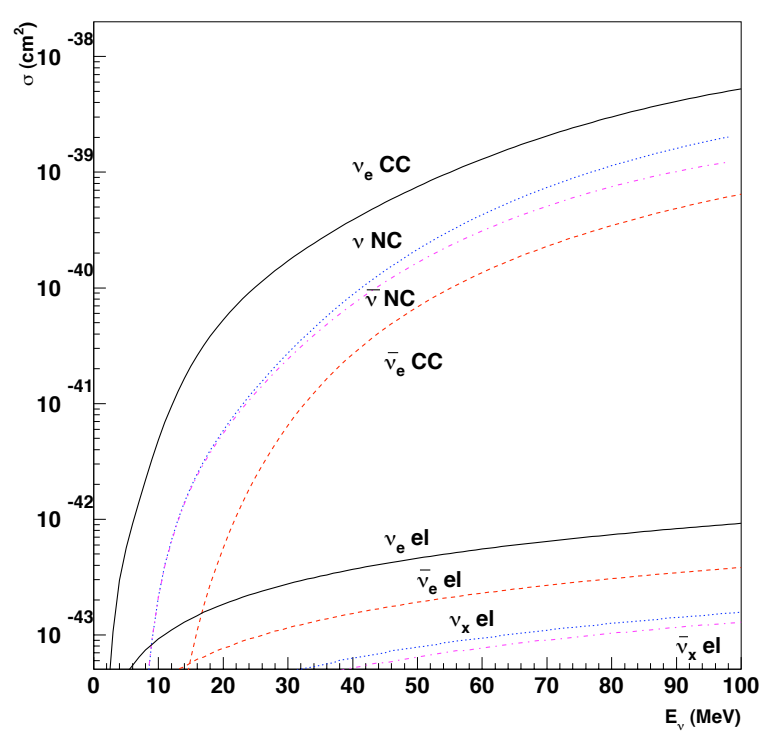

Figure 21: Cross sections for neutrinos in liquid argon, from [20]. CC (el) stands for Charged Current (elastic scattering).

- Liquid Argon (LAr). A newcomer in the panorama of neutrino detection, the liquid argon technology has only been explored at the prototype level with the 600 tons ICARUS project [146]. The new generation project DUNE will reach $\mathcal{O}(10)$ kt mass $[53,51,54]$ (Table 7). A LAr detector operates by imaging the tracks left by charged, ionizing particles, using the Time Projection Chamber (TPC) method.

\footnotetext{
${ }^{11}$ Backgrounds, of cosmogenic nature are present in scintillator, but reducible to low levels; see [58].
} 
This imaging capability - similar to that of a bubble chamber [147, 148] - allows very good particle and process identification, as well as very good energy resolution. Of the several detection processes, given in Table 7 (see fig. 21), charged current scattering of $\nu_{e}$ on the ${ }^{40} \mathrm{Ar}$ nucleus dominates, making liquid argon absolutely unique for its potential to detect the $\nu_{e}$ component of the DSNB. Neutral current scatterings on ${ }^{40} \mathrm{Ar}$ are not relevant for the detection of the DSNB because their signature - the emission of gamma rays below $11 \mathrm{MeV}$ energy from the deexcitation of the daughter nucleus - would be completely buried by solar neutrinos [20]. Scattering on electrons is subdominant due to its smaller cross section.

The energy window for the DSNB at LAr is determined by atmospheric and solar neutrinos (sec. 4.1), under the sensible assumption that other backgrounds can be effectively separated ${ }^{12}$. A full determination of backgrounds (including rare events, since the DSNB detection rate would be low) will come from extensive R\&D studies that are currently ongoing. Until then, the discussion of DSNB detection at LAr will necessarily be indicative.

\subsection{Upper limits}

\subsubsection{Data and flux constraints}

So far, the DSNB has escaped detection. Thanks to their larger volumes, currently active detectors $[8,149,150,151,152,15,16]$ have improved dramatically on the bounds set by the previous generation of experiments $[6,7]$. Table 8 summarizes the most stringent upper limits (see also fig. 1 for a graphical representation).

For a long time, the strongest bounds on all neutrino flavors were those established in 2003 by the 1496 days search at SK [8] for events with energy between the threshold of $18 \mathrm{MeV}$ (lepton energy, established after a spallation cut) and $80 \mathrm{MeV}$. The data were analyzed by the SK collaboration in the dominant detection channel, inverse beta decay induced by electron antineutrinos (Table 7). The resulting bound was $\Phi_{\bar{\nu}_{e}}(E>19.3 \mathrm{MeV})<1.2 \mathrm{~cm}^{-2} \mathrm{~s}^{-1}$ at $90 \%$ C.L., obtained by fitting the data with a single parameter, the flux

\footnotetext{
${ }^{12}$ Spallation products are expected in LAr detectors. Their presence is likely to be irrelevant for the DSNB detection, since they lie below $20 \mathrm{MeV}$ of energy, which is anyway precluded by solar neutrinos.
} 


\begin{tabular}{|c|c|c|c|c|c|}
\hline \hline Species & Experiment & reference & energy $(\mathrm{MeV})$ & limit $\left(\mathrm{cm}^{-2} \mathrm{~s}^{-1}\right)$ & C.L. \\
\hline \hline \multirow{4}{*}{$\nu_{e}$} & SK & {$[153]$} & $>19.3$ & $73.3-154$ & $90 \%$ \\
& SK (indirect) & {$[115]$} & $>19.3$ & 5.5 & $\sim 98 \%$ \\
& SNO & {$[151]$} & $22.9-36.9$ & 70 & \\
\hline \multirow{2}{*}{$\bar{\nu}_{e}$} & SK & {$[153]$} & $22.9-36.9$ & $39-54$ & $90 \%$ \\
& SK (2853 days) & {$[15]$} & $>17.3$ & $2.8-3.0$ & $90 \%$ \\
& SK (960 days) & {$[16]$} & $13.3-31.3$ & $(1.48-9.9) \times 10^{2}$ & $90 \%$ \\
& KamLAND & {$[152]$} & $8.3-31.8$ & $1.39 \times 10^{2}$ & $90 \%$ \\
& Borexino & {$[154]$} & $1.8-17.8$ & $\sim 3 \times 10^{5}$ & $90 \%$ \\
\hline$\nu_{\mu}+\nu_{\tau}$ & SK & {$[153]$} & $>19.3$ & $(1.0-1.4) \times 10^{3}$ & $90 \%$ \\
\hline $\bar{\nu}_{\mu}+\bar{\nu}_{\tau}$ & SK & {$[153]$} & $>19.3$ & $(1.3-1.8) \times 10^{3}$ & $90 \%$ \\
\hline \hline
\end{tabular}

Table 8: Summary of the most stringent bounds on the DSNB from current detectors, with their confidence level (C.L.). Unless otherwise noted, the SK bounds refer to the 1496 live days data published in [129]. The limit on the $\nu_{e}$ component labeled as "indirect" proceeds from the SK $\bar{\nu}_{e}$ limit with considerations of similarity of the $\nu_{e}$ and $\bar{\nu}_{e}$ fluxes at Earth due to neutrino oscillations in the star [115]. Where applicable, intervals of limits are given, corresponding to the range of neutrino spectra or models used in the analysis. The SNO result is also spectrum-dependent: the quoted bound is the median of several 90\% C.L. limits found with different neutrino spectra. The Borexino bound is an extrapolation from figure 2 in [154]. 
normalization. A separate, multi-parameter, study of the same data [153] in the subdominant detection channels, gave limits on the $\nu_{e}$ component of the DSNB (from $\nu_{e}$ scattering on oxygen and on electrons, Tab. 7) and on the non-electron flavors components as well (from scattering on electrons). These limits, although loose, improved on the previous constraints from SNO [151] (heavy water, $\nu_{e}$ channel) and LSD [7] (liquid scintillator, all flavors).

In 2012 the SK collaboration updated the $\bar{\nu}_{e}$ bound, giving the result in Eq. (2) [15]. This latter analysis uses 2853 days of data, is multi-parameter, and has a lower threshold, $16 \mathrm{MeV}$ of positron energy (corresponding to 17.3 MeV of neutrino energy). For comparison with earlier results, however, a limit with the $18 \mathrm{MeV}$ threshold was also given for the model in [26]: $\Phi_{\bar{\nu}_{e}}(E>19.3 \mathrm{MeV})<2.0 \mathrm{~cm}^{-2} \mathrm{~s}^{-1}$ at $90 \%$ C.L.. The reasons for obtaining a looser bound, compared to the 2003 result, are improvements in the cross section and in the statistical method used, and a (non-significant) excess in the post-2003 data [15].

A different limit on the $\bar{\nu}_{e}$ flux was obtained in 2014 by a SK search for inverse beta decay with tagging of neutron capture on hydrogen [16]. The neutron tagging allowed for a lower energy threshold, $12 \mathrm{MeV}$ of positron energy, however its efficiency is $\sim 17-18 \%$ [16]. Therefore the resulting DSNB limit is looser than that in eq. (2), but it is nevertheless interesting due to the different energy region probed.

Complementary to water results, a limit on $\bar{\nu}_{e}$ s at lower energy (threshold $7 \mathrm{MeV}$ positron energy) was established by KamLAND [149, 152], in a study originally designed mainly to constrain a possible $\bar{\nu}_{e}$ flux from the Sun. While limited by the small mass of KamLAND (1 kt), the result is an interesting test of the liquid scintillator technology, with its better signal discrimination, in anticipation of a next generation of $\mathcal{O}(10) \mathrm{kt}$ mass experiments [155]

Looking at the future, the SK collaboration plans new, improved analyses, which could further lower the energy threshold by up to $\sim 2 \mathrm{MeV}$ [137]. However, major improvements will be possible only with new concepts like Gadolinium addition, currently under study (sec. 4.5).

Aside from detailed data analyses, indirect upper bounds can be established on the basis of naturalness and theoretical considerations. In particular, a constraint on diffuse $\nu_{e}$ f follows from the constraint on the $\bar{\nu}_{e}$ component at SK by considering that the two components must be similar due to their common origin in the non-electron neutrino flavors inside the star through neutrino oscillations [115]. This is the "indirect" limit given in Table 8, and, while considerably looser than the $\bar{\nu}_{e}$ bound, it is currently the strongest for 
the $\nu_{e}$ species.

\subsubsection{Implications of the SK limit}

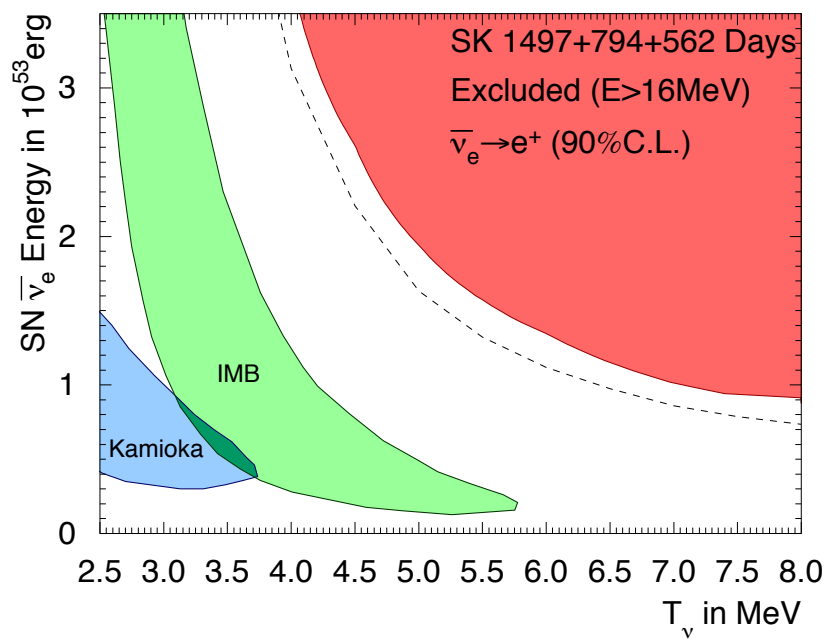

Figure 22: From [15]: $90 \%$ C.L. exclusion region from the 2011 SK analysis (upper shaded area) in the space of the total and effective temperature of the $\bar{\nu}_{e}$ flux from an individual supernova. A thermal neutrino spectrum is assumed, and the parameters $R_{-4}=1.25$, and $\beta=3.4$ (from $[156,157]$ ) were used to describe the SNR (Eq. (4)). Regions allowed by SN1987A data are shown as well (contours, originally from [158]; see also [46]). The dashed line refers to a one-dimensional analysis with the total energy $L_{\bar{e}}$ as free parameter and each value of the temperature taken as fixed.

As already observed (fig. 2), the SK limit approaches the region of theoretical predictions of the DSNB, leaving most models unconstrained. Loose constraints can be obtained in the space of parameters that describe the flux, namely the neutrino spectra and total energies, and the normalization of the SNR. Only combinations of parameters giving the highest DSNB are excluded, and generally degeneracies imply only weak constraints on individual parameters.

Two different sets of constraints are shown in figs. 22 and 23 (from $[15,43]$ ). Fig. 22 refers to fixed SNR (taken from [156]) and shows the excluded region in the space of the total energy and effective temperature of the $\bar{\nu}_{e}$ flux from an individual supernova. Parameters compatible with SN1987A are allowed, and for the theoretically natural energy of $L_{\bar{e}}=5 \cdot 10^{52}$ ergs, temperatures as high as $8 \mathrm{MeV}$ are allowed. The allowed region is expected to be even wider if uncertainties on the SNR are included. 


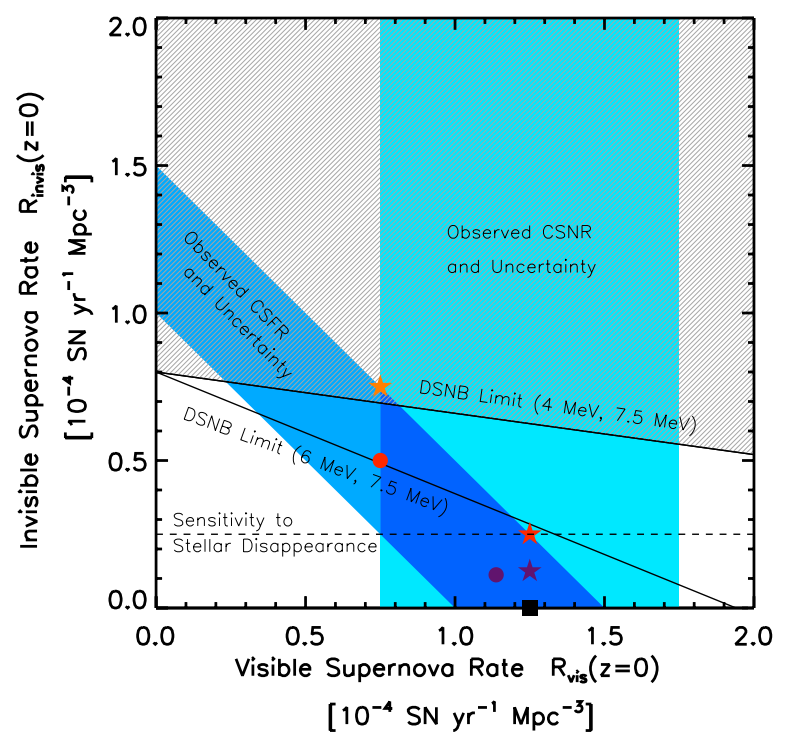

Figure 23: From [43]: exclusion region (shaded grey) from the 2003 SK limit, in the space of the cosmic rates (at $z=0$ ) of neutron star-forming (successful) and black hole-forming (failed) supernovae. For these two stellar populations, thermal neutrino spectra were used with temperatures of 4 and $7.5 \mathrm{MeV}$ respectively. A second contour gives the constraint for a different set of temperatures $(6$ and $7.5 \mathrm{MeV})$. The remaining shaded regions are those allowed by current measurements of the star formation rate (dark blue, diagonal) and of the supernova rate (light blue, vertical). The dashed line represents the projected sensitivity of future searches for failed supernovae. Stars, circles and squares refer to the parameters used in [43] for flux predictions. 
Fig. 23 instead gives the exclusion of rates of neutron star-forming (successful) and direct black hole-forming (failed) supernovae depending on the neutrino spectra for these two supernova populations, compared with astrophysical limits on the same quantities from star formation rate measurements and supernova observations. Interestingly, the rate of failed supernovae alone is constrained to be below $\sim 0.8 \cdot 10^{-4} \mathrm{yr}^{-1} \mathrm{Mpc}^{-3}$, which is however well above the rate expected for $\mathrm{a} \sim 20 \%$ fraction of failed supernovae (sec. 3.2).

While detailed analyses of parameter constraints from the SK limits have yet to be done, current results are sufficient to give a perspective of what can be expected from next generation searches (see sec. 5). With a factor of 2-4 improvement in flux sensitivity, the parameter constraints from the DSNB will become competitive with those from astronomy and from SN1987A [46].

\subsection{Water Cherenkov detectors towards megaton scale}

\subsubsection{Number of events}

After the very successful experience of the $50 \mathrm{kt}$ mass of SK, water technology is now mature to expand to megaton scale. In this section I discuss the DSNB signal for a representative setup of a $0.5 \mathrm{Mt}$ fiducial volume and 5 years running time, with energy resolution equal to that of SK (sec. 4.2). An efficiency of $93 \%[159,160]$ is used as representative of a detector performance. However one must consider that the real efficiency depends on the details of the hardware and of the data analysis and therefore results will have to be rescaled once these details are known. I include only inverse beta decay events and neglect the subdominant channels (which contribute to less than $\sim 4 \%$ [40]) and use backgrounds for the SK location, taken from [18]. To be conservative, all other specifications (like the energy threshold) will be taken to be the same as in the earliest SK analysis of [129]. This setup is necessarily indicative; some technical details will be discussed in sec. 4.4.2. All results refer to the same flux parameters as in sec. 3.5.4.

Fig. 24 shows the energy distribution of the signal and background events. It appears immediately that the signal to background ratio is always smaller than $0.2-0.3$, and is maximum just above the threshold of $18 \mathrm{MeV}$. There the signal is comparable to or larger than the atmospheric background but is exceeded by the invisible muon one. The atmospheric background starts to dominate at $\sim 25 \mathrm{MeV}$, thus closing the energy window (sec. 4.1). It appears that even for the most energetic neutrino spectrum, the peak of the event distribution is below the spallation cut, and would require a better background discrimination to be observed. 

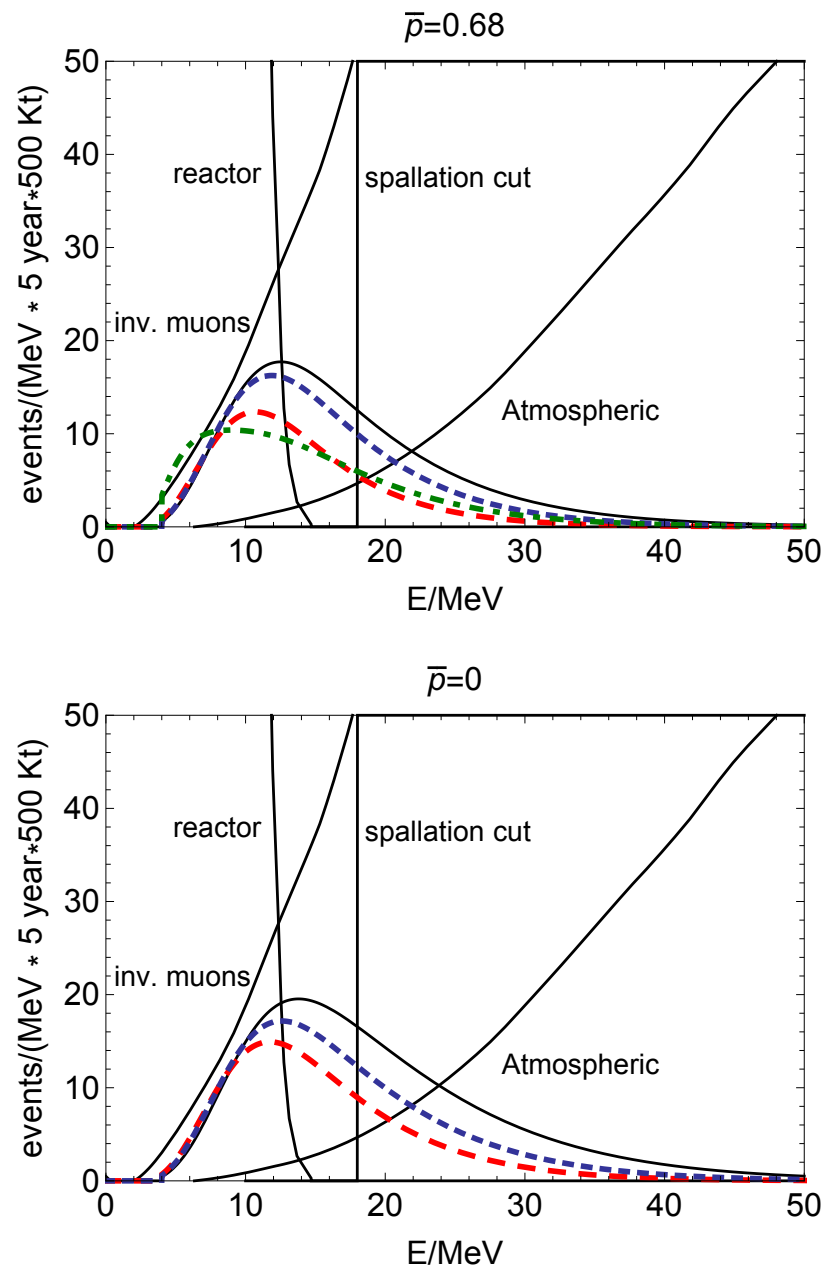

Figure 24: Energy distribution of positrons from inverse beta decay expected at a water Cherenkov detector with a $2.5 \mathrm{Mt} \cdot \mathrm{yr}$ exposure, located at Kamioka, for the two extreme values of $\bar{p}$. The solid, short dashed, long dashed and dash-dotted lines refer to the different neutrino spectra shown in fig. 15 , and specifically to the $\mathrm{H}, \mathrm{W}$ and $\mathrm{C}$ spectra, and the SN1987A best fit spectrum. The spectra of events from reactor neutrinos, atmospheric neutrinos and invisible muons are shown for comparison (from [18]). A $18 \mathrm{MeV}$ energy cut (due to spallation) is shown as well. 
Tables 9 and 10 give the numbers of signal and background events in two intervals of positron energy. While the smaller bin $\left(18<E_{e}<28 \mathrm{MeV}\right)$ is realistic and reflects the region where the signal/background ratio is maximal, the wider interval $\left(10<E_{e}<28 \mathrm{MeV}\right)$ might be relevant to a possible upgrade (for example water plus Gadolinium, sec. 4.2) where spallation is effectively removed and only reactor neutrinos remain as background at low energy.

\begin{tabular}{|c|c|c|c|c|c|c|}
\hline \hline & \multicolumn{4}{|c|}{ Signal } & \multicolumn{2}{c|}{ Background } \\
\hline & Hot & Warm & Cold & SN1987A best fit & atmosph. & inv. $\mu$ \\
\hline \hline total & 275 & 227 & 150 & 166 & & \\
\hline $10<E_{e}<28 \mathrm{MeV}$ & 203 & 167 & 103 & 102 & 115 & 1064 \\
\hline $18<E_{e}<28 \mathrm{MeV}$ & 74.5 & 53.6 & 25.8 & 34.0 & 95 & 791 \\
\hline \hline
\end{tabular}

Table 9: Numbers of signal (from inverse beta decay) and background events from atmospheric neutrinos and invisible muons in energy intervals of interest at a detector of mass $0.5 \mathrm{Mt}$ and livetime 5 years, for $\bar{p}=0.68$. The total number of signal events over the whole spectrum is also given. The neutrino fluxes in fig. 15 were used; the backgrounds are for the Kamioka site [130].

\begin{tabular}{|c|c|c|c|c|c|}
\hline \hline & \multicolumn{3}{|c|}{ Signal } & \multicolumn{2}{c|}{ Background } \\
\hline & Hot & Warm & Cold & atmosph. & inv. $\mu$ \\
\hline \hline total & 358 & 268 & 207 & & \\
\hline $10<E_{e}<28 \mathrm{MeV}$ & 259 & 199 & 151 & 115 & 1064 \\
\hline $18<E_{e}<28 \mathrm{MeV}$ & 113 & 47.9 & 73.5 & 95 & 791 \\
\hline \hline
\end{tabular}

Table 10: The same as Table 9 for $\bar{p}=0$.

From the Tables it appears that the number of signal events above 18 $\mathrm{MeV}$ varies between $\sim 20$ and $\sim 100$ depending on the neutrino energy spectra and oscillation effects. An even larger variation should be expected in consideration of the uncertainty in the normalization of the core collapse rate (sec. 3.1). The $E^{2}$ dependence of the cross section magnifies the differences due to different energy spectra of the neutrino flux, so that the event rate for the $\mathrm{H}$ spectrum can easily be twice as large as in the other cases.

To answer the question of statistical significance, one should consider the $\sim 900$ background events in the $18-28 \mathrm{MeV}$ bin, corresponding to a 

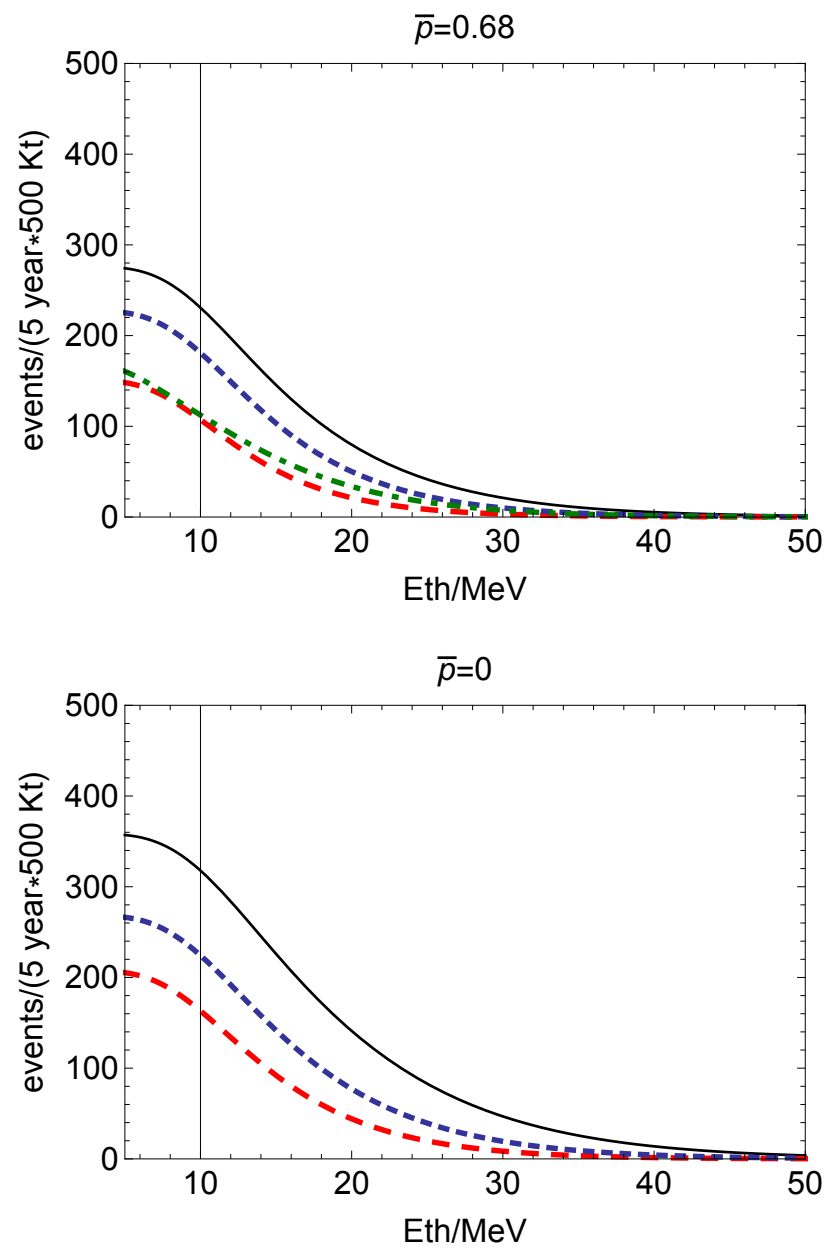

Figure 25: Inverse beta decay events above a threshold energy $E_{t h}$, as a function of $E_{t h}$ for the two extreme values of $\bar{p}$. The solid, long dashed and short dashed curves correspond to the fluxes in fig. 15 (with the same coding of colors and dashings). 
$1 \sigma$ statistical error of about 30 events. It follows that, for the exposure considered here, the signal could reach a $3 \sigma$ significance for the $\mathrm{H}$ spectrum. In general, and depending on the DSNB normalization, a longer exposure would be necessary for a high significance observation. In the worst case of $\sim 26$ signal events, an exposure 12 times longer (for example, 20 years running time for a $1.5 \mathrm{Mt}$ detector) would be required to reach a $3 \sigma$ significance ${ }^{13}$.

For an upgraded configuration with lower threshold and subtracted spallation background, the number of events from the signal can exceed 200. If the invisible muon background is subtracted as well, the signal could be comparable to or larger than the background, thus ensuring excellent statistical significance even for the most unfavorable parameters. This is a very strong motivation for improvements in this direction. A discussion of possibilities is given in sec. 4.5.

For generality, I also give numbers of signal events above a range of thresholds, in fig. 25. They can be used to calculate the event rates in other energy bins of interest. For selected thresholds the figure also gives the $90 \%$ C.L. interval predicted by imposing compatibility with SN1987A and with direct measurements of the supernova rate [21] (sec. 3.1 and 3.4). One can see the substantial decline in the event rate with the increase of the threshold beyond the peak of the DSNB ( $E \sim 6-7 \mathrm{MeV}$, see sec. 3.2).

The diffuse flux, and therefore the event rate, could be substantially enhanced if failed supernovae are relatively numerous ( $\sim 20 \%$ or so) in the universe, as discussed in sec. 3.2. Figure 26 gives the expected energy distribution and integrated rate of events expected in the best case scenario of fig. 17 (largest failed supernova flux, with S EoS, $\bar{p}=0.68$ and $f_{N S}=0.79$ ). It appears that above $18 \mathrm{MeV}$ of positron energy the events due to failed supernovae could amount to about $2 / 3$ of the total, enhancing the rate up to about 200 events for an exposure of $2 \mathrm{Mt} \cdot \mathrm{yr}$. This enhancement could allow an earlier detection at $3 \sigma$ of the DSNB, already with half the exposure. Thanks to the more energetic spectrum of failed supernovae, lowering the energy threshold to $14 \mathrm{MeV}$ or so would be sufficient to capture the bulk of

\footnotetext{
${ }^{13}$ Notice that these considerations on statistical significance assume that the normalization of the background is well know independently, which is not the case at this time. If poorly constrained, this normalization will be included in the data analysis as a fit parameter, as is currently done by the SK collaboration $[129,15]$, and this weakens the sensitivity to the signal.
} 


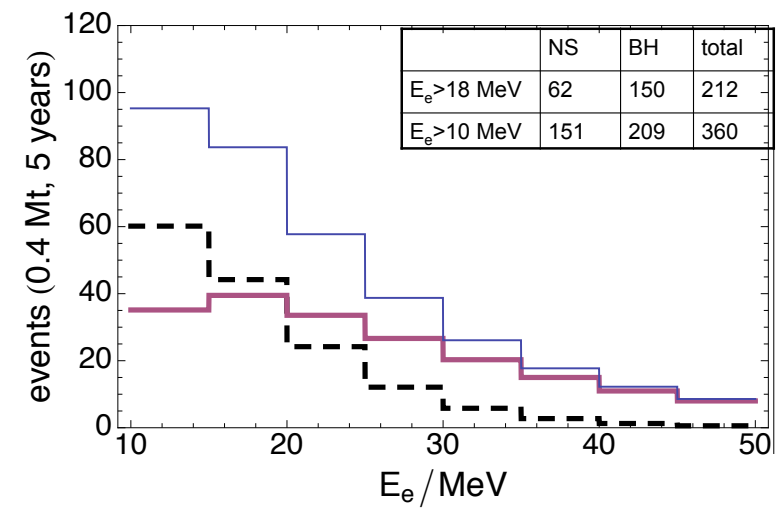

Figure 26: From [42]: events in water from direct black hole-forming collapses (solid thick), from neutron star-forming collapses (dashed) and the total of the two (thin line) for the best case scenario in fig. 17 (largest failed supernova flux, with the $\mathrm{S} \operatorname{EoS}, \bar{p}=0.68$ and $\left.f_{N S}=0.78\right)$. A $2 \mathrm{Mt} \cdot \mathrm{yr}$ exposure is used; $E_{e}$ is the positron energy. The inset gives the number of events above selected thresholds.

the events from these objects and see the peak of their event distribution. With a $10 \mathrm{MeV}$ threshold the number of signal events can exceed 300.

\subsubsection{Technical considerations}

Certainly the event rates given here are indicative, since they ultimately depend on the specific detector design. For example, the energy resolution and the fiducial volume (for a given total mass) are influenced by the detector's geometry, photocathode coverage, electronics, etc.. As discussed in sec. 4.1, location and depth influence the backgrounds importantly. Choosing the technical specifications of an experiment always involve balancing scientific, financial, technical and logistical requirements. Here I discuss some of the currently favored setups in their main physics aspects.

- geometry. The excavation of a cavern large enough to contain a single, undivided, $\sim 1$ Mt mass of water is certainly challenging but possible [161]. However, current designs favor a modular approach, with the detector consisting of multiple separate volumes. An example is the proposed two-volume layout for Hyper-Kamiokande (fig. 27) [48] (see 
also $[162,49]$ for different concepts), where considerations of rock stability suggest a quasi-cylindrical design for each module. Compared to a single volume, modularity would result in a smaller fiducial volume (for equal total mass), but is attractive because it breaks several challenges (excavation, instrumentation, maintenance, etc..) into more manageable parts that can be completed sequentially or in parallel depending on circumstances. The modular design also shortens the waiting time, since it can start to deliver data as soon as the first module is completed, similarly to what happened for another supermassive project, IceCube [163].

For Mt masses, the same photocatode coverage of SK (40\%) may be unrealistic, and therefore a lower coverage (20-30\%) is envisioned for at least part of the detector [48]. This would result in a slight worsening of the low energy performance $(\sim 7 \mathrm{MeV}$ rather than $\sim 5 \mathrm{MeV}$ threshold, which is however inconsequential for the energy window of the DSNB), as well as a somewhat poorer energy resolution [164], with consequent poorer background discrimination and ultimately higher energy threshold for DSNB searches.

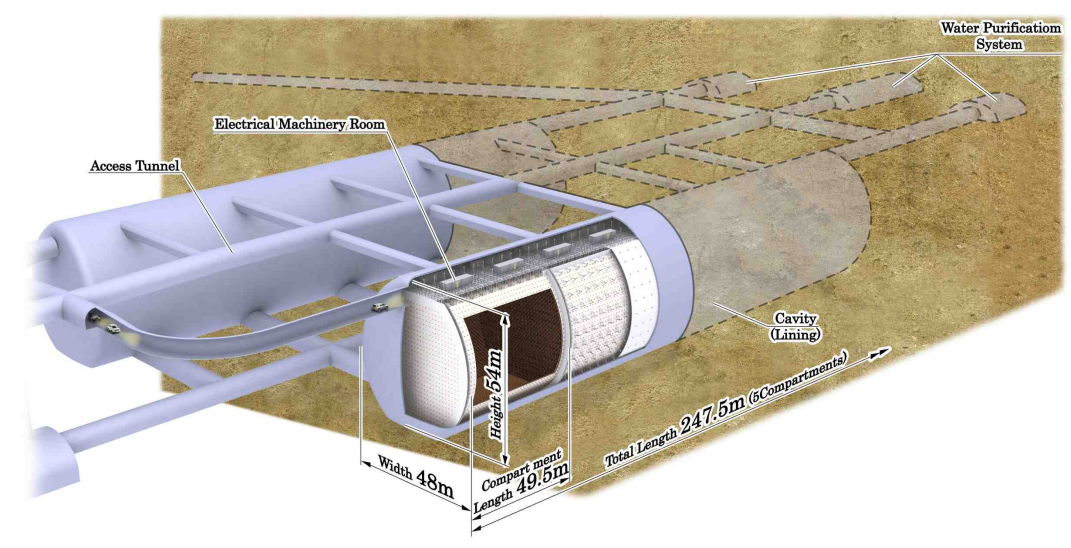

Figure 27: Conceptual design of Hyper-Kamiokande, [48].

- depth. Since the cosmic ray flux in a detector rapidly decreases with 
depth, even modest gains in depth result in substantially enhanced performance. This is especially true for the DSNB, for which detection is dramatically limited at low energy by spallation products of cosmic ray muons. A detailed study on depth requirements for a Mt Cherenkov detector [165], gives the expected extension of the energy window with the increase of depth, relative to that of SK $(3300 \mathrm{ft}$, equivalent to $1005 \mathrm{~m}$ and to $2900 \mathrm{~m}$ water-equivalent). Results are given in Table 11. For pure water, by going to $4850 \mathrm{ft}$ depth the energy window can be pushed down to $15.5 \mathrm{MeV}$ of positron energy, with an enhancement of the DSNB signal of about $40 \%$. This depth is also required to have

\begin{tabular}{|l|l|l|l|}
\hline $\begin{array}{l}\text { rock depth } \\
\text { ft. }\end{array}$ & $\begin{array}{l}\text { water equiv } \\
\text { km-w-e }\end{array}$ & $\begin{array}{l}\text { Energy thres. without } \\
\text { (with) Gd }(\mathrm{MeV})\end{array}$ & $\begin{array}{l}\text { Signal rate without (with) } \\
\text { Gd relative to } 18 \mathrm{MeV}\end{array}$ \\
\hline 4850 & 4.3 & $15.5(12.0)$ & $1.4(2.0)$ \\
3500 & 3.1 & $17.5(15.0)$ & $1.1(1.5)$ \\
3300 & 2.9 & $18.0(15.5)$ & $1.0(1.4)$ \\
2000 & 1.8 & $20.5(18.0)$ & $0.73(1.0)$ \\
300 & 0.27 & $25.0(22.5)$ & $0.36(0.55)$ \\
\hline
\end{tabular}

Table 11: Expected energy threshold for a water Cherenkov detector with and without Gd addition as a function of depth for detection of the DSNB, from [165]. The DSNB spectrum used in [165] is close to the $\mathrm{H}$ spectrum (but with slightly hotter $\nu_{x}, E_{0 x}=21.6$ $\mathrm{MeV}$ ) with $\bar{p}=0.68 \quad$ (see sec. 3.2).

sensitivity to the day-night asymmetry of solar neutrinos [166].

- Location/latitude Even though latitude is important for the atmospheric neutrino background, considerations of depth always prevail in the choice of a particular underground location, together with considerations like access of people and technology to the site. Although several sites have been considered recently for a Mt-scale detector (Table 6), at this time efforts are concentrated on the Hyper-Kamiokande project in Japan [48].

\subsection{Reducing backgrounds with liquid scintillator and Gadolinium}

\subsubsection{SuperK-Gd}

The idea of dissolving gadolinium in water was proposed by Beacom and Vagins [57], who discussed its cost-effectiveness and non-toxicity. They envisioned applying the idea to the SK detector, thus initiating a new phase 
of SK called SuperK-Gd ${ }^{14}$. This initiative is especially attractive for its relatively low cost and short timeline; in July 2015, it has been officially approved by the SK collaboration [167].
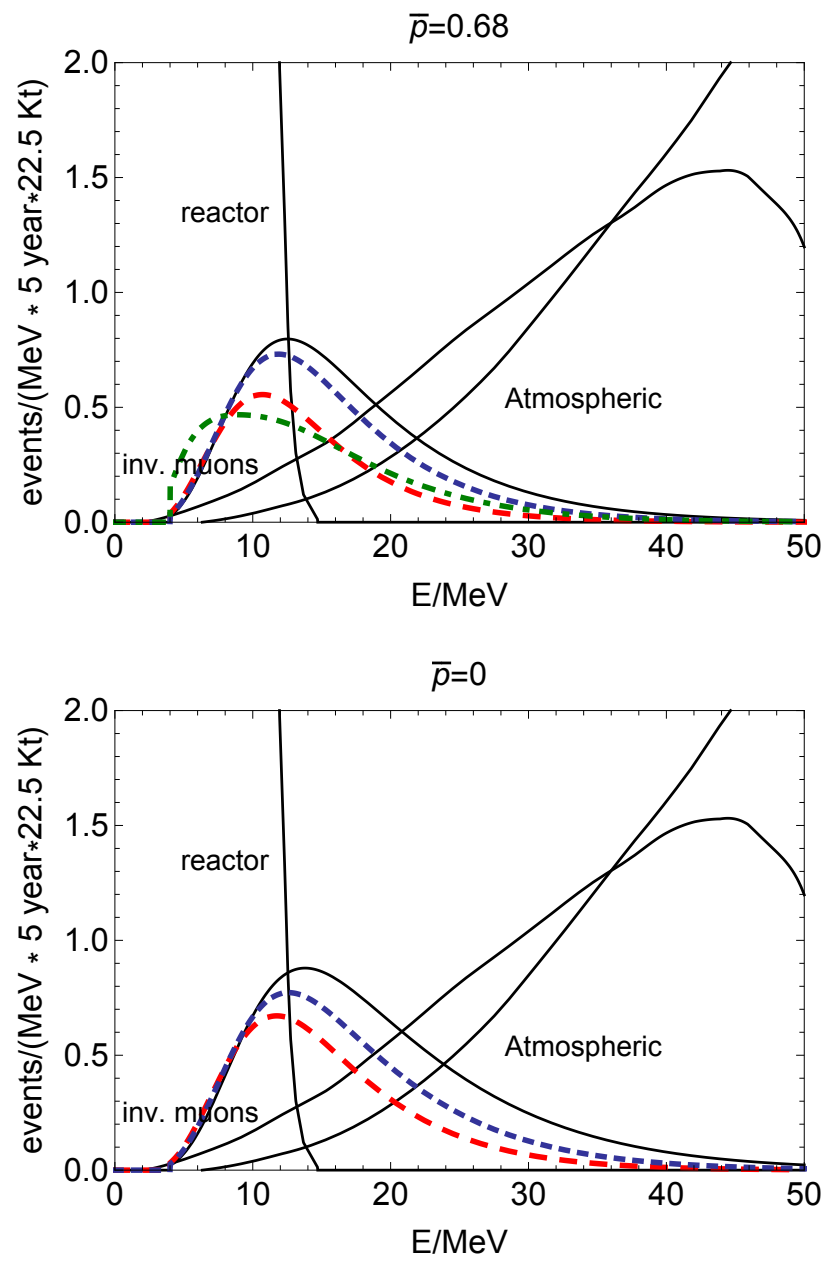

Figure 28: The same as fig. 24 for SuperK-Gd (22.5 kt water with Gd). Notice the strong reduction of the invisible muon background.

It is estimated that, with Gd, the background due to spallation will be subtracted almost completely and the one due to invisible muons will be

\footnotetext{
${ }^{14}$ In the original work by Beacom and Vagins [57], and until recently, this upgrade was called GADZOOKS! "Gadolinium Antineutrino Detector Zealously Outperforming Old Kamiokande, Super!".
} 
reduced by a factor of $\sim 5$ [57]. As a result, the lower end of the energy window for DSNB detection is determined by reactor neutrinos, and would typically be $11-12 \mathrm{MeV}$ in neutrino energy. In the interval $\sim 12-20 \mathrm{MeV}$ the signal dominates over the background. This is illustrated in fig. 28, where the enlarged energy window appears, possibly including (for the $\mathrm{H}$ spectrum ) the peak of the event energy distribution.

The number of events expected at SuperK-Gd can be evaluated by rescaling the event rates of Tables 9 and 10 by the appropriate volume factor. Assuming 10 years running time and an indicative $22.5 \mathrm{kt}$ fiducial volume one expects 9 - 28 signal events and 29 background events in the window of $10-28 \mathrm{MeV}$ of positron energy. Thus, the excess due to the DSNB would be statistically significant for part of the parameter space, and the significance will likely be enhanced by the spectral analysis of the data. However, detailed studies of the signal properties would probably be beyond reach.

The recent intense R\&D work on SuperK-Gd focused on finding a Gd compound that meets four basic criteria: (i) solubility, (ii) limited impact on water transparency, (iii) negligible reactions with the detector materials (e.g., corrosion, etc.) and (iv) compatibility with a detector's filtration system ${ }^{15}$.

Initially $\mathrm{GdCl}_{3}$ was considered [168], but was then discarded due to poor performance in water transparency, and since then a $0.2 \%$ solution of Gd sulfate $\left(\mathrm{Gd}_{2}\left(\mathrm{SO}_{4}\right)_{4}\right)$ has emerged as the favorite option, as it meets the criteria outlined above and is safe for stainless steel tanks [169].

This compound is now being tested further at EGADS ("Employing Gadolinium to Autonomously Detect Supernovas"), a dedicated 200 t prototype at Kamioka, where data-taking is in progress $[170,171]^{16}$.

\subsubsection{Liquid scintillator at the $20 \mathrm{kt}$ scale}

Liquid scintillator detectors of tens of kt mass have been envisioned for at least a decade. Initial studies focused on a $50 \mathrm{kt}$ configuration, the european LENA (Low Energy Neutrino Astronomy) [155], whose potential

\footnotetext{
${ }^{15} \mathrm{~A}$ detector like SK requires constant purification of the water to avoid deterioration of its transparency. It is necessary to prove that a Gd compound can be effectively filtered and reinserted into the detector. Another requirement is the feasibility to completely remove the Gd compound from the water to restore the detector to a pure water phase.

${ }^{16}$ Other important questions have to be addressed before SuperK-Gd can be realized: one of them is environmental concerns (real or perceived by the public opinion), related to the existence of a leak in the SK tank. I leave this aspect to more specialized literature (e.g., [172]).
} 
for the DSNB was assessed in dedicated studies [58, 50]. Lately, upcoming projects have settled on a more realistic $\sim 20 \mathrm{kt}$ mass, with the Asia-based JUNO (Jiangmen Underground Neutrino Observatory, in China) [55] and RENO-50 (Reactor Experiment for Neutrino Oscillation, in South Korea) [56]. For definiteness, here I consider the parameters for JUNO : $17 \mathrm{kt}$ fiducial volume, corresponding to $N_{p}=1.23 \cdot 10^{33}$ protons [55]. When appropriate, a number of early results of the LENA collaboration will be adapted to the current context, as they are still the most detailed in many respects. Thanks to the detection in coincidence of the positron and neutron capture (sec. 4.2), the detection of diffuse $\bar{\nu}_{e} \mathrm{~s}$ at JUNO will be similar to that at SuperK-Gd : the spallation and invisible muon backgrounds are effectively subtracted, so the energy window is determined by reactor and atmospheric neutrinos and extends from $\sim 10 \mathrm{MeV}$ to $\sim 28 \mathrm{MeV}$. The signal event rate will be similar as well, considering that the number of protons in JUNO is only $22 \%$ smaller than that of SK/SuperK-Gd $\left(N_{p}=1.5 \cdot 10^{33}\right)$. Therefore about $8-24$ events are expected in 10 years at JUNO.

The relevant backgrounds for liquid scintillator are currently under study. Well understood backgrounds are the inverse beta decay events due to atmospheric and reactor neutrinos, and to a residual spallation product, fast neutrons [58]. They contribute to $\sim 11$ events per decade [55]. Additional backgrounds are due to $\beta n$ emitters (mainly ${ }^{9} \mathrm{Li}$ ) [58, 50], and to neutral current scattering of atmospheric neutrinos [50]. The latter is especially dangerous, with a rate that exceeds the signal by about one order of magnitude [173]. However, subtraction techniques are being studied, and, preliminary results on pulse-shape discrimination indicate that it might be possible to achieve a signal-to-background ratio larger than $1[50,174,55]$.

The current design of JUNO is shown in fig. 29 [55]. It is characterized by a spherical geometry, with the inner detector surrounded by a water Cherenkov volume acting as muon veto. A 70-80\% wall coverage of photomultipliers is envisioned. The detector will be located in Jinji town, in the Guangdong province of China; its construction is now approved by the Chinese Academy of Sciences, and it is expected to start taking data in 2020 $[55]$.

\subsection{Liquid argon for $\nu_{e}$ detection}

\subsubsection{Numbers of events}

From the intense scoping work of the past years it has been recognized that a mass of several tens of kilotons is necessary for non-beam physics with 


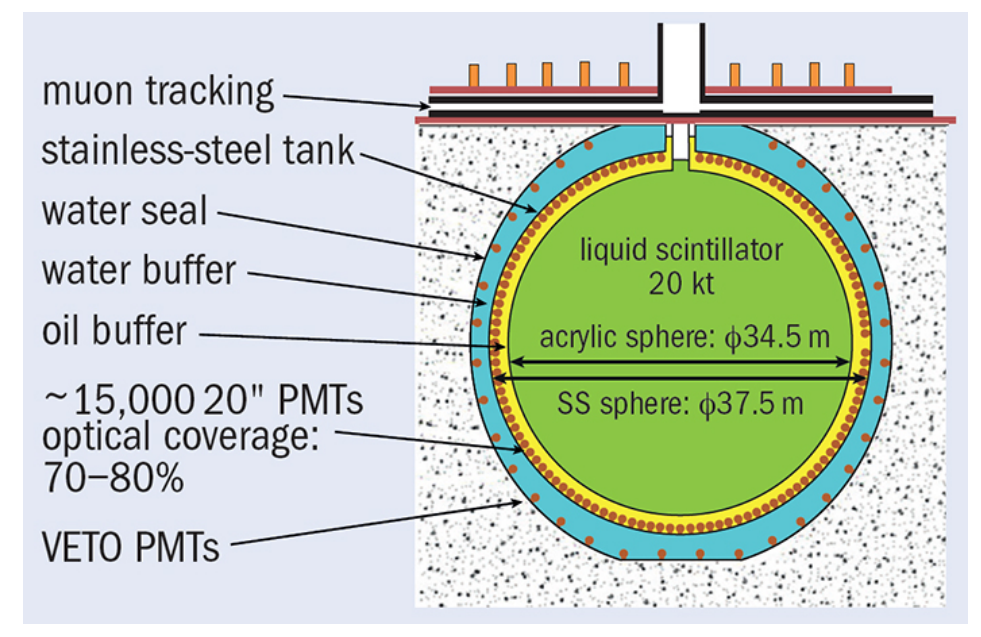

Figure 29: JUNO conceptual design, from [175].

liquid argon. This scale will be reached by the USA-EU joint project DUNE - to be built in the Homestake mine, South Dakota, USA - for which $40 \mathrm{kt}$ fiducial mass is envisioned in its full configuration [54]. Here this mass is considered; and a running time of 5 years is chosen for illustration. Here I include only the dominant process, charged current $\nu_{e}$ interaction with argon, (Table 7). For this, the energy of the emitted electron differs from that of the incoming neutrino by $\sim 3-4 \mathrm{MeV}$ depending on the nuclear transition taking place [20]. Since detailed information on the spectrum of these transitions is not available, however, here event rates will be discussed in terms of neutrino energy. All results refer to the diffuse flux as in fig. 15.

Fig. 30 shows the distribution of signal and backgrounds in neutrino energy, for the extreme values of the survival probability $p$. The signal peaks between 12 and $18 \mathrm{MeV}$ depending on the oscillation scenario and on the original neutrino spectra. At the peak, $\sim 0.3-0.6$ events $/ \mathrm{MeV}$ are expected. Backgrounds dominate below $\sim 19 \mathrm{MeV}$ and above 24-32 MeV depending on the parameters. This energy window is similar to that of a water detector (fig. 24). Unlike the case of water, however, here it may happen (for the most optimistic parameters) that the peak of the signal might fall near or inside the energy window. This is thanks to the faster rise of the cross section with energy, compared to inverse beta decay.

Tables 12 and 13 give the number of events from the signal and from the atmospheric background in energy intervals of interest. In the $19-39 \mathrm{MeV}$ 

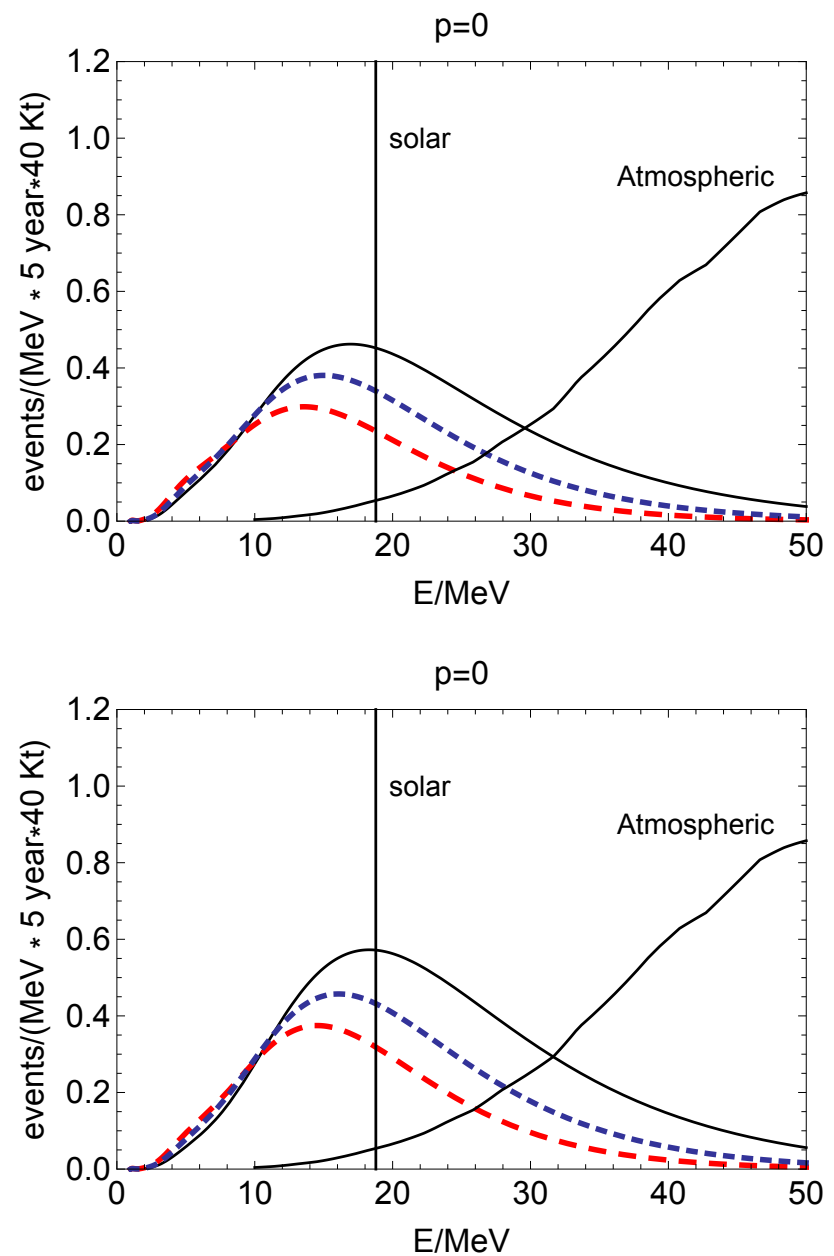

Figure 30: Distribution of charged current $\nu_{e}$ events in liquid argon (Table 7) in neutrino energy for a $40 \mathrm{kt}$ LAr detector located at Homestake for the different models of $\nu_{e}$ diffuse flux given in fig. 15 (same dashings and color coding), and the two extreme values of $p$. The spectra of events from the relevant backgrounds are shown for comparison. 


\begin{tabular}{|c|c|c|c|c|}
\hline \hline & Hot & Warm & Cold & atmospheric \\
\hline \hline $19<\mathrm{E} / \mathrm{MeV}<29$ & 3.6 & 2.3 & 1.4 & 1.3 \\
\hline $19<\mathrm{E} / \mathrm{MeV}<39$ & 5.3 & 3.2 & 1.9 & 5.2 \\
\hline $5<\mathrm{E} / \mathrm{MeV}<39$ & 9.9 & 7.3 & 5.3 & 5.4 \\
\hline \hline
\end{tabular}

Table 12: Rate of charged current $\nu_{e}$ interactions on ${ }^{40} \mathrm{Ar}$ (Tab. (7)) in three energy windows of interest (given in terms of the neutrino energy, $E$ ) at a liquid argon detector of mass $40 \mathrm{kt}$ and livetime 5 years, for $p=0.32$. All flux parameters are as in fig. 15 . The atmospheric background is for the Homestake location, and is expected to be a factor of $\sim 1.5$ lower at Kamioka, see fig. 18 .

\begin{tabular}{|c|c|c|c|c|}
\hline \hline & Hot & Warm & Cold & atmospheric \\
\hline \hline $19<\mathrm{E} / \mathrm{MeV}<29$ & 4.8 & 3.1 & 2.0 & 1.3 \\
\hline $19<\mathrm{E} / \mathrm{MeV}<39$ & 7.3 & 4.3 & 2.6 & 5.2 \\
\hline $5<\mathrm{E} / \mathrm{MeV}<39$ & 12.3 & 8.9 & 6.7 & 5.4 \\
\hline \hline
\end{tabular}

Table 13: Same as Table 12 for $p=0$.

window the detector may register between $\sim 3$ and 5 events of signal and $\sim 5$ events of atmospheric background. The signal increases with the average energy of the $\nu_{e}$ flux entering the detector, and therefore is higher for the $\mathrm{H}$ spectrum and for $p=0$ (complete swap between the original $\nu_{e}$ and $\nu_{x}$ fluxes). Even in the most favourable case, a longer exposure (at least 20 years) would be required to reach a $3 \sigma$ statistical significance of the signal. A similar result holds for a narrower energy window, $19-29 \mathrm{MeV}$, where the increase in the signal to background ratio is roughly compensated by the decrease in statistics. One should remember, however, that the overall normalizations of the DSNB and of the atmospheric neutrino flux are highly uncertain, and therefore the signal to background ratio could very well be a factor of a few larger for all models, thus leading to more encouraging results.

Tables 12 and 13 also give numbers of events in a larger energy window, 5 - $39 \mathrm{MeV}$, which might be applicable if a method is found to subtract events from solar neutrinos, probably on the basis of directional information from kinematics reconstruction. This possibility has not been investigated so far, and so, while speculative, it remains open. The Tables show that accessing 
lower energies might enhance the event rate by up to a factor of 3 for the models with lower neutrino average energy.
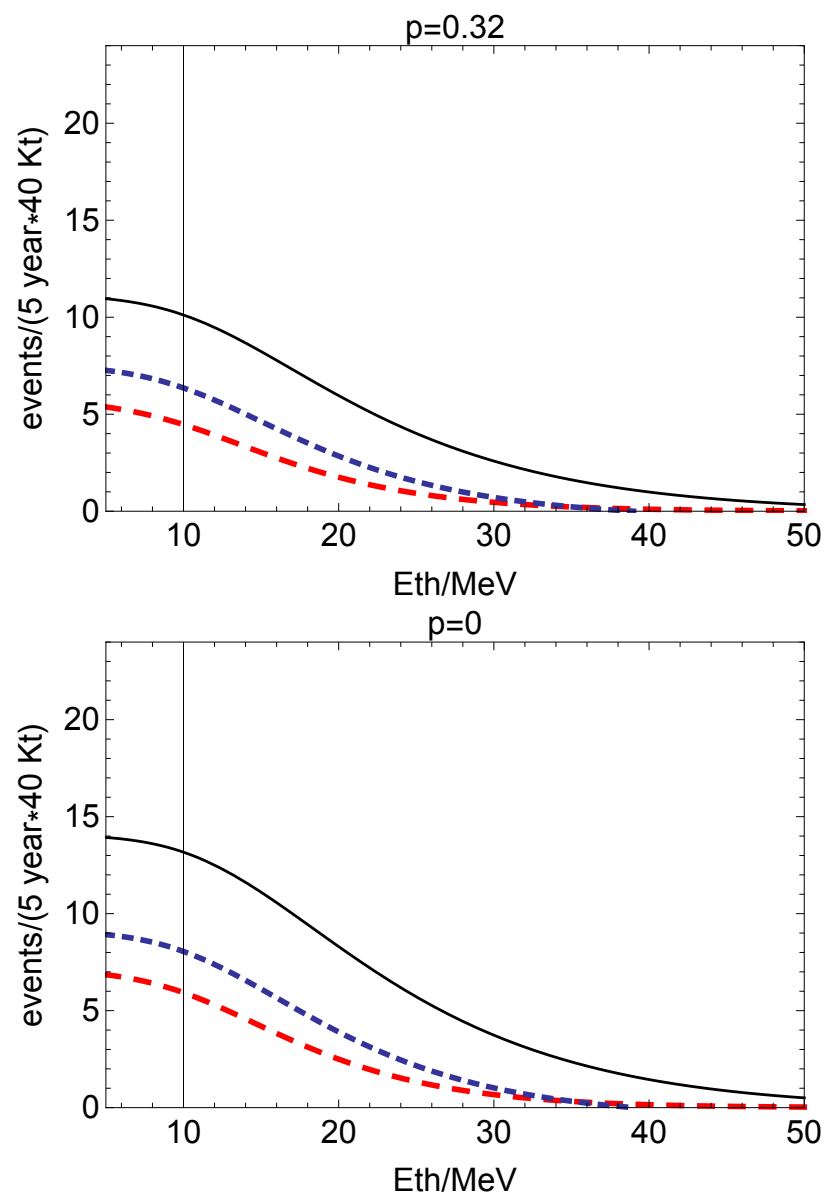

Figure 31: $\nu_{e} \mathrm{CC}$ events in liquid argon above a certain neutrino energy $E_{t h}$, as a function of $E_{t h}$ for the two extreme values of $p$. The solid, short dashed and long dashed curves are from the $\mathrm{H}, \mathrm{W}$ and $\mathrm{C}$ spectra examples (see Table 1). All other parameters are as in fig. 30 .

For completeness, fig. 31 gives the numbers of DSNB events above a certain neutrino energy $E_{t h}$, as a function of $E_{t h}$.

Similarly to water detectors, also for LAr the event rate could be enhanced by failed supernovae. Fig. 32 illustrates this, by showing the number and energy distribution of events from normal and failed supernovae for the most optimistic parameters (largest failed supernova flux, with the S EoS, $p=0.32$ 


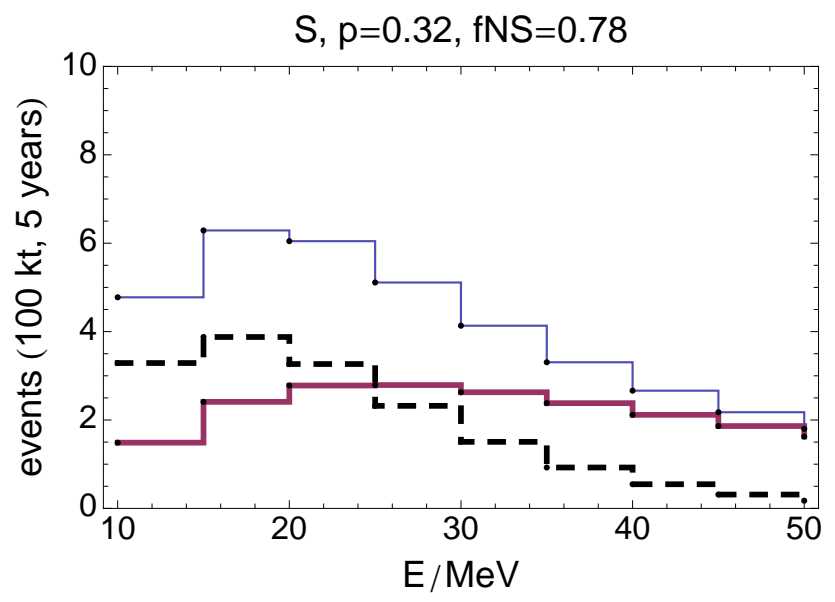

Figure 32: From [92]: events in LAr from black hole-forming collapses (solid thick), from neutron star-forming collapses (dashed) and the total of the two (thin line) for the parameters that maximize the failed supernova flux (S EoS, $p=0.32$ and $f_{N S}=0.78$, see sec. 3.5.5). Events are plotted in bins of neutrino energy. Note the long exposure: $500 \mathrm{kt} \cdot \mathrm{yr}$, equivalent to 12.5 years of operation for DUNE.

and $f_{N S}=0.78$, sec. 3.5.5). It appears that the contribution of failed supernovae peaks in the energy window and enhances the event rate by a factor of $\sim 2$ in the same energy interval. This implies a higher chance that a LAr experiment might see an indication of signal in its earliest phase of operation.

\subsection{2. technical considerations}

The technical realization of the liquid argon concept at $40 \mathrm{kt}$ mass is still a subject of discussion. Current research is focused on testing the performance of liquid argon in lower mass projects, on designing the necessary electronics, and on the engineering of the final, full scale, structure and its accommodation underground. Essential criteria are the scalability (in mass) of the design and safety in operations of handling and containing the argon under pressure.

Current low mass efforts that are specific for neutrino physics include ICARUS $[146,177,178]$, MicroBOONE $[179,180]$ (now under construction), and ArgoNEUT [181, 182] (running).

Detailed designs for DUNE already exist; fig. 33 illustrates a project for a $10 \mathrm{kt} \mathrm{LAr}$ module, functioning as a time-projection chamber (TPC), where the readout anode is composed of wire planes inside the LAr volume. 


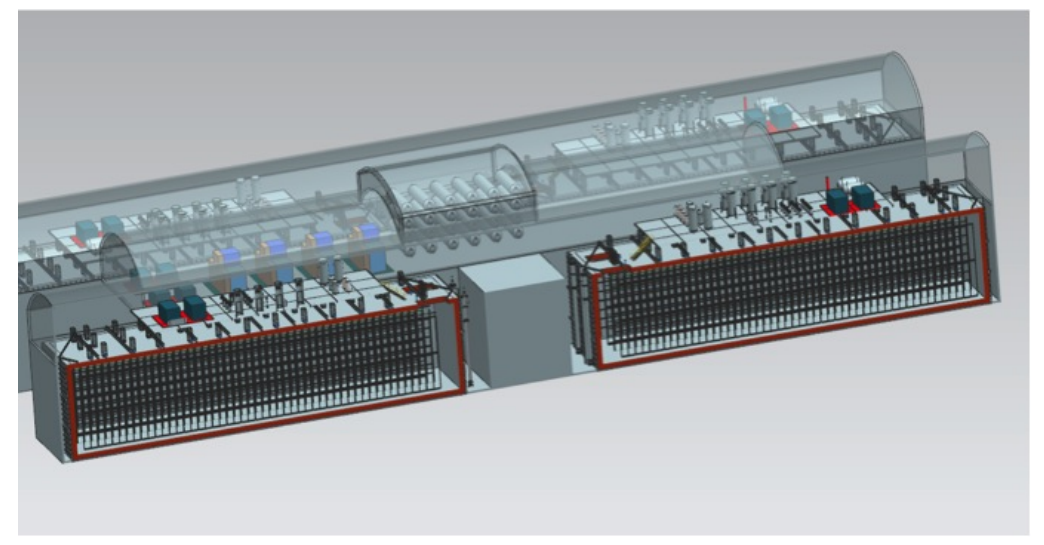

Figure 33: Conceptual design of a $10 \mathrm{kt}$ module of the US-based DUNE detector [54]. This is the proposed single phase solution; an alternative dual phase design is also considered, see [54] for details. The full DUNE detector will be made of four modules.

An alternative concept is characterized by a dual phase, with the ionization charges being detected in a layer of gaseous Argon above the liquid phase. See [54] for more details.

\section{Perspectives: what can be learned and how?}

The question of what can be learned from data on the DSNB has been explored only partially so far. It is reasonable to expect that, as data start to appear, there will be a first phase of analysis that will constrain the basic ingredients of the DSNB at the coarse level, with errors of the order of 50$100 \%$ due to backgrounds and parameter degeneracies. What these basic ingredients are emerges from the previous sections: the neutrino spectra in the different flavors, conversion/survival probabilities, and normalization parameters like the total energy emitted in neutrinos. These are discussed in sec. 5.1 and 5.2. Exotic physics and subdominant effects are briefly discussed in sec. 5.3 and 5.4; directions for improvements and final considerations then follow to conclude this section.

\subsection{Testing the physics of supernovae: neutrino spectra}

Naturally, the sensitivity of an experiment to the DSNB spectrum depends critically on its energy window. Limited sensitivity is expected for pure water and LAr, where the window is only 10-15 MeV wide, while liquid scintillator and SuperK-Gd-type (water+Gd) designs should perform better 
thanks to the larger window. Detailed studies have been done for water, water $+\mathrm{Gd}$, and liquid scintillator. Most of them discussed the dependence of event rates on the neutrino spectra (e.g, [18, 22, 40]), while the question of parameter degeneracy has been addressed only in part [128, 25, 33, 31].

One way to eliminate the degeneracy with normalization parameters $\left(L_{\bar{e}}\right.$, $L_{\bar{x}}$ and $R_{-4}$ ), is to study ratios of events, for example the ratio $r$ of the number of events in the first and second energy bin $[128]^{17}$. Taking bins of 5 $\mathrm{MeV}$ width, this ratio is:

$$
r \equiv \frac{N\left(18 \leq E_{e} / \mathrm{MeV}<23\right)}{N\left(23 \leq E_{e} / \mathrm{MeV}<28\right)},
$$

for the water only case, and

$$
r \equiv \frac{N\left(10 \leq E_{e} / \mathrm{MeV}<15\right)}{N\left(15 \leq E_{e} / \mathrm{MeV}<20\right)}
$$

for water $+\mathrm{Gd}$, in terms of the positron energy $E_{e}$.

Fig. 34 [128] shows isocontours of $r$ in the space of the spectral parameters $\alpha_{w}-E_{0 w}$, for fixed SNR and in the assumption that the spectral form in Eq. (6) applies to the $\bar{\nu}_{e}$ flux at Earth.

For pure water $r$ varies in the range $r \simeq 1.5-4.3$. For a $2 \mathrm{Mt} \times \mathrm{yr}$ exposure, the error on $r$ is larger than $\sim 100 \%$ due to the high background rate [128]; this confirms the conclusion that the sensitivity to the neutrino spectrum is limited for pure water. For the configuration with extended energy window (water+Gd) one gets $r \simeq 1-2.5$. Thanks to the better background reduction, the error is down to $\sim 20-30 \%$, meaning that at least the extreme cases should be distinguishable, for typical values of the normalization parameters.

If in the future the normalizations and the background fluxes become known precisely, the number of signal events $N$ in the energy window can be used jointly with the ratio $r$ to constrain the spectral parameters. Isocontours of $N$ are given in fig. 34; they intersect the isocontours of $r$, suggesting that a joint measurement of $N$ and $r$ could be more constraining than the two separately.

\footnotetext{
${ }^{17}$ Including higher energy bins enhances the backgrounds more than the signal and therefore it worsens the sensitivity to the neutrino spectrum [128].
} 

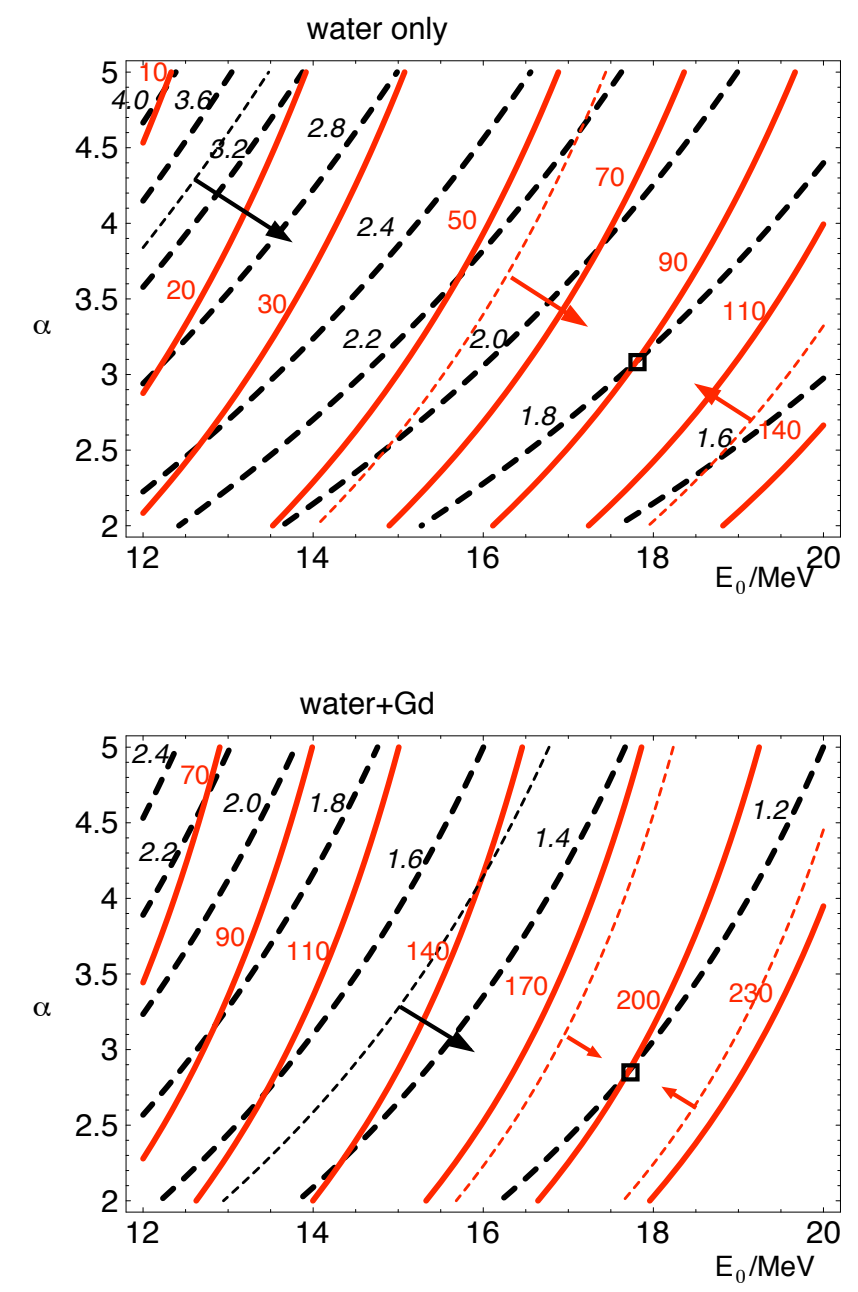

Figure 34: From [128]: isocontours of the numbers of events $N$ (solid red lines), and of the ratio $r$ of events in the first two energy bins (Eqs. (20) and (21), long dashed black lines), at a water Cherenkov detector with $1.8 \mathrm{Mt} \times \mathrm{yr}$ exposure, in the space of the spectral parameters $\alpha_{w}-E_{0 w}$ (in the assumption that the spectral form in Eq. (6) applies to the $\bar{\nu}_{e}$ flux at Earth). In each panel a possible measurement of $N$ and $r$ is shown, with central values (diamond) and $1 \sigma$ statistical errors (regions within the short dashed lines, marked with arrows; the wider region is the error on $r$ ). The SNR parameters and total energy emitted in neutrinos are as in fig. 15. 
In essence, the results in fig. 34 tell us that data will allow us to reconstruct the spectrum of the neutrino emitted by a supernova in terms of two effective parameters, an average energy and a shape parameter. The next step of the analysis would be to reconstruct the more fundamental quantities that determine the effective ones, such as the neutrino spectra in the different flavors at the production point, and the neutrino mixings and mass hierarchy. This second level of detail is certainly more challenging, and is likely to require a combination of precision-phase experiments and improved theoretical modeling. An example of this is given in a detailed study [58] for a liquid scintillator detector with an exposure of $\sim 400 \mathrm{kt} \times \mathrm{yr}$ (equivalent to about 24 years of running for JUNO). Considering the number of events in two different energy bins, and including neutrino oscillations, it was found that different spectrum models can be discriminated with $\sim 2-2.5 \sigma$ confidence level. This conclusion is only moderately encouraging, and may become weaker when it is updated with a full inclusion of all the backgrounds (see sec. 4.5.2).

\subsection{Testing neutrino flavor conversion}

The sensitivity of the DSNB to neutrino oscillation effects, and therefore to the mass hierarchy and to the phenomenology of collective oscillations (sec. 3.3), remains an open question. Many studies have included conversion effects in predictions of the DSNB, examining their impact on the rates and energy distributions of events at detectors (sec. 4). Effects are strong for both quantities, however, what exactly can be concluded on oscillation parameters is not clear, in consideration of the many degeneracies. Specifically, event rates have a degeneracy with the normalization parameters, while energy distributions are meaningful only with priors (or a measurement from a galactic supernova) on the originally emitted neutrino spectra in the different flavors. A detailed reconstruction of spectral shapes might resolve this degeneracy to some extent, but only for the larger energy windows of SuperK-Gd and of a liquid scintillator detector, provided that they run for at least a decade to accumulate sufficient statistics.

Some of the degeneracies can be resolved by the combination of several complementary datasets. For example, a set of data from $\nu_{e}$ and another from $\bar{\nu}_{e}$ would help to eliminate degeneracies with the normalization parameters and would allow to probe the permutation parameters $p$ and $\bar{p}$. This can lead to the discrimination of the mass hierarchy, as already observed for an individual supernova (e.g., $[112,183,184]$ ). For the DSNB, this step of 
connecting measured probabilities with the fundamental oscillation parameters might be complicated by the integration over the diverse supernova population; therefore it might be advisable to focus on probability determination, with the understanding that the measured probabilities should be intended as effective/averaged parameters. The connection with the swap probabilities $P_{c}, \bar{P}_{c}$, and with the mass hierarchy ${ }^{18}$ may be attempted if the formulae in Table 2 are assumed to apply for the DSNB.

\subsection{Sensitivity to neutrino exotica}

Analogously to what happened with SN1987A, the data from the diffuse supernova neutrinos will test innumerable new physics hypotheses -typically the existence of new particles and/or new forces - from searches of unexpected features in the neutrino signal.

The sensitivity of the DSNB to these exotica has been studied only minimally. In view of the substantial cosmological contribution to it (see sec. 3.5.3), one expects the DSNB to be particularly constraining, compared to SN1987A, of phenomena that require large propagation distances, such as effects of decay or absorption of the neutrinos during their propagation across the universe.

Effects of neutrino decay on the DSNB were studied by [37, 38]. Depending on the neutrino mass spectrum, the decay can enhance or suppress the electron flavor component, in a way that could be observable for ratios of the neutrino lifetime over mass as high as $\tau / m \simeq 10^{10} \mathrm{~s} / \mathrm{eV}$ (fig. 35). One should be mindful, however, of the possible degeneracy with the normalizations of the neutrino flux and of the SNR.

Exotic absorption effects could be caused by a high rate of neutrinoantineutrino annihilation in the cosmological relic neutrino background, mediated by new light gauge bosons [39], or by resonant neutrino-dark matter scattering [41].

About non-propagation (between source and detector) effects, it has been shown that resonant spin flavor conversion inside the star influences the ex-

\footnotetext{
${ }^{18}$ Rigorously, the mass hierarchy is the only independent parameter that is still unknown. In principle, $P_{c}$ and $\bar{P}_{c}$ are completely determined by neutrino masses and mixings, as well as by neutrino flavor spectra and total energies at production. However, they are also very sensitive to subtle and poorly known effects of non-linearity and symmetry breaking. For this reason, it may be practical to consider them as independent parameters in a data analysis.
} 


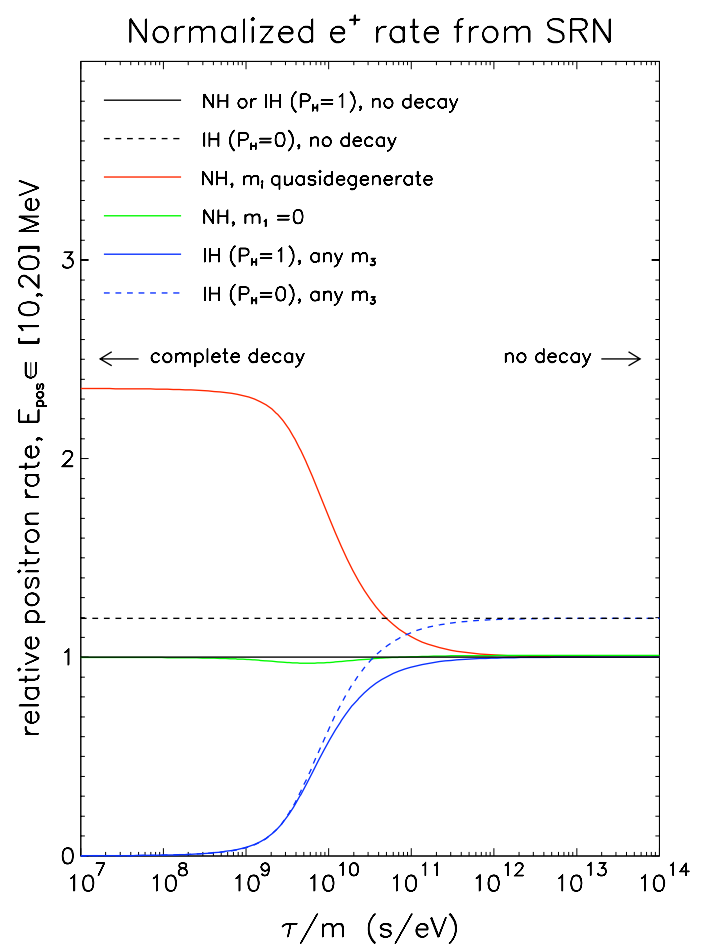

Figure 35: From [38]: positron event rates in water in the energy range [10,20] MeV for various decay scenarios, relative to the case of no decay with $\bar{p} \simeq 0.68$ (solid horizontal line). 
pected event rates [40]. It has also been observed that the DSNB could in principle provide a test of dark energy, complementary to astrophysical measurements [185].

Besides affecting the DSNB, physics beyond the Standard Model could also mimic it under certain circumstances. These could be the annihilation or decay of light dark matter into neutrinos of energy comparable to the DSNB (see e.g. $[186,187,188]$ ). A flux similar to the DSNB could be made of solar antineutrinos, if their production is enhanced by resonant spin-flavor conversion [189]. Ultimately, when detectors reach the precision phase, it will be possible to distinguish the DSNB from other, non-supernova fluxes, on the basis of spectral and/or directional information. For the first detection phase, however, it is likely that a complete disambiguation might not be possible, so that a first detection of a diffuse flux will have multiple interpretations.

\subsection{Subdominant effects}

For completeness, it is worth mentioning other phenomena that will be difficult to study with the DSNB, either due to their modest $(\sim 10 \%$ effect or less) impact, or due to background. They might become of interest for an advanced precision phase of the DSNB in the more distant future.

- regeneration of $\nu_{e}$ and $\bar{\nu}_{e}$ due to oscillations inside the Earth. In the DSNB these effects are below 5\% [115] and therefore statistically insignificant.

- the swap in the neutrino energy spectrum due to neutrino-neutrino refraction (sec. 3.2). The spectral step caused by the swap for a single supernova will be smoothed out by the integration over the redshift and by the presence of individual differences between supernova progenitors $[24,25]$. Besides, for part of the parameter space, the swap occurs below $10 \mathrm{MeV}$ of energy, and thus is likely to fall outside the energy window for DSNB detection.

- the SNR at large redshift, $z \gtrsim 1$. This will be difficult to probe because neutrino produced at large redshifts accumulate at low energy, where backgrounds dominate (see sec. 3.5.3).

- the normalization of the supernova rate. This is degenerate with the neutrino flux normalization. Probably, it will be better constrained by astronomical surveys of supernovae. 
- shockwave effects on the neutrino spectrum due to oscillations. First pointed out in [113], these effects are very interesting probes of oscillation parameters as well as of the physics of the shock. Once integrated over the duration of a neutrino burst, and over a diverse population of stars at different redshifts, they reduce to a $\sim 10 \%$ size or less $[17,23]$, with no distinctive spectral features in the DSNB. Therefore, they are likely to be indistinguishable from many other effects of similar magnitude.

- the time structure of neutrino emission from a supernova, including the neutronization burst, the accretion phase and the cooling phase, with their characteristic time scales $[25,33]$. The DSNB is a sum of many time-integrated supernova bursts, which causes loss of information.

\subsection{Directions for improvement}

\subsubsection{Phenomenology of the DSNB}

The directions in which phenomenology can develop are many. Here I outline some that I consider most important to advance the field.

- Contribution of different supernova types. Perhaps, the truly unique value of the DSNB - in comparison to neutrinos from individual supernovae - is its sensitivity to the diverse stellar population that contributes to it, which spans decades of progenitor masses and different stellar properties (magnetic fields, metallicities, etc.). A comprehensive study of how this diversity is reflected in the DSNB would be extremely important to fully explore the physics potential of this flux. It would require a systematic modeling of the neutrino emission for a large variety of supernova progenitors, a goal that is certainly ambitious but realistic, in principle. At this time, numerical simulations exist for selected values of the progenitor mass, and some $[83,86]$ ) have been used for initial studies on the DSNB $[25,33]$. Results indicate that the effect of the progenitor mass distribution is of the order of $10 \%$ or less.

- from one supernova to many: what does really matter? As commented previously, when summing over many sources and including redshift effects, some loss of information is unavoidable. It would be desirable to have a systematic study of the features and size of each different effect (e.g., oscillations, propagation effects, time structures, etc.). An initial study obtained with a specific set of numerical flux predictions [25] 
shows that the MSW-driven flavor conversion may dominate over other effects like spectrum cooling and the dependence on the progenitor mass. However, it is possible that, in certain circumstances, several small effects may sum to a sizable contribution, with no single one of them dominating. Other literature discusses the dependence on the stellar dynamics; the effect of the time of shock revival was found to be of the order of tens of per cent [33]. A description at such level of detail might be premature at this time, but will be needed when the precision phase begins.

- What fundamental physics will we learn? Related to the previous point is the question of how much information will be possible to extract from the DSNB data on specific quantities of interest, like the SNR, neutrino spectra, oscillation parameters, etc. This question is closely connected to that of experimental capability, and requires a careful study of how data can be processed for best parameter extraction. It will be of immediate relevance after the DSNB is first detected.

In addition to these directions, it is expected that studies of the DSNB will be updated over time, following the developments in the physics of neutrinos and of supernovae. New, more precise determinations of the SNR will be used after a new phase of supernova surveys, some of which are already taking data e.g., [197, 198, 199, 200, 201]. A more detailed description of oscillation effects will have to be adopted once the phenomenology of neutrino self-interactions (sec. 3.3) is completely understood. Progress in the determination of oscillation parameters - especially if the mass hierarchy is established - will help to converge towards a uniquely determined oscillation scenario. Finally, as the modeling of neutrino emission and transport in supernovae progresses, updated neutrino fluxes will be available with a reduced uncertainty on the neutrino flavor spectra and total energies.

If a galactic supernova is observed in the next decade or so, the study of the DSNB will take a different turn. The abundance of data from the single nearby supernova will eliminate or greatly reduce the uncertainty on the original neutrino fluxes and on oscillations. This will allow more precise predictions for the DSNB, with more focus on its sensitivity to the supernova population of the universe and a number of cosmological and astrophysical effects. 


\subsubsection{Experimental searches}

From what was discussed so far, one can see that the experimental study of the DSNB has two strong limiting factors: the detector mass and the backgrounds.

Increasing the mass of a detector is of course desirable for a higher statistical significance of a DSNB signal. At the moment, any expansion beyond $\sim 1 \mathrm{Mt}(\sim 50 \mathrm{kt})$ mass for water Cherenkov (LAr) seems speculative. However, there a conceptual study has been presented for a $5 \mathrm{Mt}$ mass water Cherenkov detector to be built under sea, off the coast of Japan [190]. The sensitivity of this design to supernova neutrinos has been studied at a basic level [191], but many questions of technical nature remain open. In particular, the larger volume is likely to come at the price of worse performance in terms of energy threshold, background, etc., in a way that remains to be assessed. Similarly, a $\sim 10 \mathrm{Mt}$ under-ice detector has been suggested as an upgrade of the IceCube detector [192]. Although a rough similarity with an under-sea concept is expected, for this proposal the sensitivity to the DSNB has not been determined yet.

For currently planned detectors, efforts to better discriminate the backgrounds at low energy, and therefore expand the energy window, are in progress (sec. 4). Even though it might be possible to gain 2-3 MeV by purely technical upgrades, a new approach might be needed to discriminate backgrounds that are orders of magnitude larger than the signal, like solar neutrinos for LAr detectors or reactor antineutrinos for water and liquid scintillator. In the absence of concrete ideas, here I outline some possible directions for further thinking, some of which are purely speculative at the moment.

- directional detection. Reconstructing the direction of arrival of neutrinos and antineutrinos in a detector would be a very effective way to discriminate the solar and reactor backgrounds. Unfortunately, the relevant detection processes have weak correlation between the direction of the neutrino and that of the daughter lepton at the energies of interest (see e.g., [138]). Therefore the complete kinematics of the process would need to be reconstructed for directional sensitivity. This seems unrealistic for water and LAr detectors [139]. Studies in the context of geoneutrinos show that some directional information can be extracted by adding ${ }^{6} \mathrm{Li},{ }^{10} \mathrm{~B}$ or Gadolinium to liquid scintillator [193, 194, 140], with ${ }^{6} \mathrm{Li}$ emerging as the best candidate $[194,140]$. It is not clear, 
however, if the improvement in directionality would be sufficient to separate reactor neutrinos at the level needed to study the DSNB.

- turning off or optimizing nuclear reactors. Considering the current efforts in developing renewable energy, it is conceivable that in the far future fission reactors will be abandoned. The absence of reactor neutrinos would allow a sensitivity to the $\bar{\nu}_{e}$ component of the DSNB down to at least $4.5 \mathrm{MeV}$, where the flux of geoneutrinos terminates $[5]^{19}$. This implies the possibility to study the peak of the DSNB, which would allow a detailed reconstruction of the neutrino spectrum and would provide precious information on supernovae at large redshift $(z>1$, see sec. 3.5.3). If a new generation of fission reactors is built, instead, its flux might be reduced by negotiating down times with the reactor managements, or even by negotiating a reactor-free zone within a certain distance from major detectors. Negotiations could prove unreliable, however. Interestingly, one could minimize the reactor flux also by arranging the detector-reactor distance to maximize the suppression effect of oscillations, which can be as large as $\left(1-\sin ^{2} 2 \theta_{12}\right) \simeq 0.13$. This could be done either by planned construction of detectors and reactors, or with a movable detector [196].

- extraterrestrial detectors. In the light of a renewed interest in manned exploration of space, one can dream that a few centuries from now underground laboratories might exist on the moon and/or on Mars or other planets of the solar system. Even without a colonization of them, scientific labs may flourish there the same way they do in Antarctica today, even though with the new challenges of extraterrestrial conditions. A large neutrino detector on Mars, for example, would benefit from the lower solar flux and from the suppressed atmospheric neutrino flux reflecting the thinner atmosphere. Reactor neutrinos might be absent there if power is provided by alternative forms of energy (fusion, solar, etc.). Of course, investing in such detector would require a strongly motivated and diverse scientific agenda, which might include studying the radioactivity of the planet with geoneutrinos.

\footnotetext{
${ }^{19}$ In fact, such situation has already been realized, with the temporary general shutdown of nuclear reactors in Japan, following the Fukushima nuclear accident in 2011. This allowed a background-free study of geoneutrinos [195].
} 


\subsection{Final considerations}

At the opening of a new phase of neutrino observatories , the possibility to observe diffuse supernova neutrinos has a quiet appeal of its own, that well complements the thrill of playing the lottery of galactic supernovae. As new detectors turn on, the DSNB could be the first new signal to emerge, nicely sandwiched between higher precision data from known sources at low and high energy. This is a guaranteed flux that must be there - even though with a large normalization uncertainty - and once detected, it will mark the transition of supernova neutrinos from rare events (SN1987A) to everyday physics, like solar and atmospheric neutrino have been in the past several years.

Over decades, the DSNB will go through the phases of discovery, maturity and precision, delivering unique information on the population of core collapse supernovae all the way to cosmological distances, and thus complementing information from individual supernovae on the physics of core collapse and on the properties of the neutrino as a particle. In addition, there might be surprises: unexpected turns of which neutrino physics has been rich so far.

\section{Acknowledgments}

I am indebted to Alessandra Tonazzo, Michael Smy, Mark Vagins and Michael Wurm for important comments and feedback; many thanks to Shunsaku Horiuchi, James Keehn, Orlando L. G. Peres and Lili Yang for useful discussions. I acknowledge the support of the NSF under Grant No. PHY0854827 and PHY-1205745.

[1] K. Eguchi et al. [KamLAND Collaboration], Phys. Rev. Lett. 90, 021802 (2003) [hep-ex/0212021].

[2] N. Arnaud, M. Barsuglia, M. A. Bizouard, V. Brisson, F. Cavalier, M. Davier, P. Hello and S. Kreckelbergh et al., Astropart. Phys. 21, 201 (2004) [gr-qc/0307101].

[3] S. Ando, J. F. Beacom and H. Yuksel, Phys. Rev. Lett. 95, 171101 (2005) [astro-ph/0503321].

[4] G. S. Bisnovatyi-Kogan and Z. F. Seidov, Soviet Astronomy (Tr: A. Zhurn.) 26, 132, 1982. 
[5] L. M. Krauss, S. L. Glashow and D. N. Schramm, Nature 310, 191 (1984).

[6] W. Zhang, E. W. Beier, L. Feldscher, E. D. Frank, W. Frati, S. B. Kim, A. K. Mann and F. M. Newcomer et al., Phys. Rev. Lett. 61, 385 (1988).

[7] M. Aglietta, P. Antonioli, G. Badino, G. Bologna, C. Castagnoli, A. Castellina, V. L. Dadykin and W. Fulgione et al., Astropart. Phys. 1, 1 (1992).

[8] M. Malek et al. [Super-Kamiokande Collaboration], Phys. Rev. Lett. 90, 061101 (2003) [hep-ex/0209028].

[9] T. Totani and K. Sato, Astropart. Phys. 3, 367 (1995) [astro$\mathrm{ph} / 9504015]$.

[10] S. E. Woosley, J. R. Wilson, and R. Mayle, Astrophys. Jour., Part 1 302, 19 (1986).

[11] T. Totani, K. Sato and Y. Yoshii, Astrophys. J. 460, 303 (1996) [astro$\mathrm{ph} / 9509130]$.

[12] R. A. Malaney, Astropart. Phys. 7, 125 (1997) [astro-ph/9612012].

[13] D. H. Hartmann and S. E. Woosley, Astropart. Phys. 7, 137 (1997).

[14] M. Kaplinghat, G. Steigman and T. P. Walker, Phys. Rev. D 62, 043001 (2000) [astro-ph/9912391].

[15] K. Bays et al. [Super-Kamiokande Collaboration], Phys. Rev. D 85, 052007 (2012) [arXiv:1111.5031 [hep-ex]].

[16] H. Zhang et al. [Super-Kamiokande Collaboration], Astropart. Phys. 60, 41 (2015) [arXiv:1311.3738 [hep-ex]].

[17] S. Ando and K. Sato, Phys. Lett. B 559, 113 (2003) [astro-ph/0210502].

[18] G. L. Fogli, E. Lisi, A. Mirizzi and D. Montanino, JCAP 0504, 002 (2005) [hep-ph/0412046].

[19] S. Ando and K. Sato, New J. Phys. 6, 170 (2004) [astro-ph/0410061]. 
[20] A. G. Cocco, A. Ereditato, G. Fiorillo, G. Mangano and V. Pettorino, JCAP 0412, 002 (2004) [hep-ph/0408031].

[21] C. Lunardini, Astropart. Phys. 26, 190 (2006) [astro-ph/0509233].

[22] S. Chakraborty, S. Choubey, B. Dasgupta and K. Kar, JCAP 0809, 013 (2008) [arXiv:0805.3131 [hep-ph]].

[23] S. Galais, J. Kneller, C. Volpe and J. Gava, Phys. Rev. D 81, 053002 (2010) [arXiv:0906.5294 [hep-ph]].

[24] S. Chakraborty, S. Choubey and K. Kar, Phys. Lett. B 702, 209 (2011) [arXiv:1006.3756 [hep-ph]].

[25] C. Lunardini and I. Tamborra, JCAP 1207, 012 (2012) [arXiv:1205.6292 [astro-ph.SR]].

[26] S. Ando, K. Sato and T. Totani, Astropart. Phys. 18, 307 (2003) [astro$\mathrm{ph} / 0202450]$.

[27] L. E. Strigari, M. Kaplinghat, G. Steigman and T. P. Walker, JCAP 0403, 007 (2004) [astro-ph/0312346].

[28] S. Ando, Astrophys. J. 607, 20 (2004) [astro-ph/0401531].

[29] F. Iocco, G. Mangano, G. Miele, G. G. Raffelt and P. D. Serpico, Astropart. Phys. 23, 303 (2005) [astro-ph/0411545].

[30] F. Daigne, K. A. Olive, P. Sandick and E. Vangioni, Phys. Rev. D 72, 103007 (2005) [astro-ph/0509404].

[31] G. J. Mathews, J. Hidaka, T. Kajino and J. Suzuki, Astrophys. J. 790, 115 (2014) [arXiv:1405.0458 [astro-ph.CO]].

[32] S. Horiuchi, J. F. Beacom and E. Dwek, Phys. Rev. D 79, 083013 (2009) [arXiv:0812.3157 [astro-ph]].

[33] K. i. Nakazato, Phys. Rev. D 88, no. 8, 083012 (2013) [arXiv:1306.4526 [astro-ph.HE]].

[34] M. Fukugita and M. Kawasaki, Mon. Not. Roy. Astron. Soc. 340, L7 (2003) [astro-ph/0204376]. 
[35] H. Yuksel and J. F. Beacom, Phys. Rev. D 76, 083007 (2007) [astro$\mathrm{ph} / 0702613$ [ASTRO-PH]].

[36] F. Vissani and G. Pagliaroli, Astron. Astrophys. 528, L1 (2011) [arXiv:1102.0447 [astro-ph.HE]].

[37] S. Ando, Phys. Lett. B 570, 11 (2003) [hep-ph/0307169].

[38] G. L. Fogli, E. Lisi, A. Mirizzi and D. Montanino, Phys. Rev. D 70, 013001 (2004) [hep-ph/0401227].

[39] H. Goldberg, G. Perez and I. Sarcevic, JHEP 0611, 023 (2006) [hep$\mathrm{ph} / 0505221]$.

[40] C. Volpe and J. Welzel, arXiv:0711.3237 [astro-ph].

[41] Y. Farzan and S. Palomares-Ruiz, JCAP 1406, 014 (2014) [arXiv:1401.7019 [hep-ph]].

[42] C. Lunardini, Phys. Rev. Lett. 102, 231101 (2009) [arXiv:0901.0568 [astro-ph.SR]].

[43] A. Lien, B. D. Fields and J. F. Beacom, Phys. Rev. D 81, 083001 (2010) [arXiv:1001.3678 [astro-ph.CO]].

[44] H. Yuksel and M. D. Kistler, arXiv:1212.4844 [astro-ph.HE].

[45] L. E. Strigari, J. F. Beacom, T. P. Walker and P. Zhang, JCAP 0504, 017 (2005) [astro-ph/0502150].

[46] H. Yuksel, S. Ando and J. F. Beacom, Phys. Rev. C 74, 015803 (2006) [astro-ph/0509297].

[47] C. Lunardini, Nucl. Phys. Proc. Suppl. 168, 131 (2007).

[48] K. Abe, T. Abe, H. Aihara, Y. Fukuda, Y. Hayato, K. Huang, A. K. Ichikawa and M. Ikeda et al., arXiv:1109.3262 [hep-ex].

[49] L. Agostino et al. [MEMPHYS Collaboration], JCAP 1301, 024 (2013) [arXiv:1206.6665 [hep-ex]].

[50] M. Wurm et al. [LENA Collaboration], Astropart. Phys. 35, 685 (2012) [arXiv:1104.5620 [astro-ph.IM]]. 
[51] A. Stahl, C. Wiebusch, A. M. Guler, M. Kamiscioglu, R. Sever, A. U. Yilmazer, C. Gunes and D. Yilmaz et al., CERN-SPSC-2012-021.

[52] C. Adams et al. [LBNE Collaboration], arXiv:1307.7335 [hep-ex].

[53] C. Baltay et al., Particle Physics Project Prioritization Panel (P5), 2014, available at http://science.energy.gov/hep/hepap/reports/

[54] M. Bishai et al., Long-Baseline Neutrino Facility (LBNF) and Deep Underground Neutrino Experiment (DUNE) Conceptual Design Report, Vol. 1, 2015. Available at http://lbne2-docdb.fnal.gov/cgibin/ShowDocument?docid $=10687$

[55] F. An et al. [JUNO Collaboration], arXiv:1507.05613 [physics.ins-det].

[56] S. B. Kim, arXiv:1412.2199 [hep-ex].

[57] J. F. Beacom and M. R. Vagins, Phys. Rev. Lett. 93, 171101 (2004) [hep-ph/0309300].

[58] M. Wurm, F. von Feilitzsch, M. Goeger-Neff, K. A. Hochmuth, T. M. Undagoitia, L. Oberauer and W. Potzel, Phys. Rev. D 75, 023007 (2007) [astro-ph/0701305].

[59] E. E. Salpeter, Astrophys. J. 121, 161 (1955).

[60] S. E. Woosley, A. Heger and T. A. Weaver, Rev. Mod. Phys. 74, 1015 (2002).

[61] A. M. Hopkins and J. F. Beacom, Astrophys. J. 651, 142 (2006) [astro$\mathrm{ph} / 0601463]$.

[62] S. Cole et al. [2dFGRS Collaboration], Mon. Not. Roy. Astron. Soc. 326, 255 (2001) [astro-ph/0012429].

[63] M. T. Botticella, M. Riello, E. Cappellaro, S. Benetti, G. Altavilla, A. Pastorello, M. Turatto and L. Greggio et al., Astron. Astrophys. 479, 49 (2008) [arXiv:0710.3763 [astro-ph]].

[64] E. Cappellaro, R. Evans and M. Turatto, Astron. Astrophys. 351, 459 (1999) [astro-ph/9904225]. 
[65] W. Li, R. Chornock, J. Leaman, A. V. Filippenko, D. Poznanski, X. Wang, M. Ganeshalingam and F. Mannucci, arXiv:1006.4613 [astroph.SR].

[66] J. Melinder, T. Dahlen, L. M. Trinchant, G. Ostlin, S. Mattila, J. Sollerman, C. Fransson and M. Hayes et al., arXiv:1206.6897 [astro-ph.CO].

[67] O. Graur, D. Poznanski, D. Maoz, N. Yasuda, T. Totani, M. Fukugita, A. V. Filippenko and R. J. Foley et al., arXiv:1102.0005 [astro-ph.CO].

[68] G. Bazin et al. [SNLS Collaboration], arXiv:0904.1066 [astro-ph.CO].

[69] T. Dahlen, L. G. Strolger, A. G. Riess, S. Mattila, E. Kankare and B. Mobasher, Astrophys. J. 757, 70 (2012) [arXiv:1208.0342 [astroph.CO]].

[70] L. Yang, PhD thesis, Arizona State University, 2013. Available at http://repository.asu.edu/items/20985 .

[71] L. Yang and C. Lunardini, in preparation.

[72] S. J. Smartt, J. J. Eldridge, R. M. Crockett and J. R. Maund, arXiv:0809.0403 [astro-ph].

[73] S. Horiuchi, J. F. Beacom, C. S. Kochanek, J. L. Prieto, K. Z. Stanek and T. A. Thompson, Astrophys. J. 738, 154 (2011) [arXiv:1102.1977 [astro-ph.CO]].

[74] M. T. Keil, G. G. Raffelt and H. T. Janka, Astrophys. J. 590, 971 (2003) [astro-ph/0208035].

[75] C. J. Horowitz, Phys. Rev. D 65, 043001 (2002) [astro-ph/0109209].

[76] T. Totani, K. Sato, H. E. Dalhed and J. R. Wilson, Astrophys. J. 496, 216 (1998) [astro-ph/9710203].

[77] T. A. Thompson, A. Burrows and P. A. Pinto, Astrophys. J. 592, 434 (2003) [astro-ph/0211194].

[78] C. Y. Cardall, A. O. Razoumov, E. Endeve and A. Mezzacappa, astro$\mathrm{ph} / 0510704$. 
[79] O. Papish, J. Nordhaus and N. Soker, arXiv:1402.4362 [astro-ph.SR].

[80] A. Marek and H.-T. Janka, Astrophys. J. 694, 664 (2009) [arXiv:0708.3372 [astro-ph]].

[81] C. D. Ott, A. Burrows, L. Dessart and E. Livne, Astrophys. J. 685, 1069 (2008) [arXiv:0804.0239 [astro-ph]].

[82] L. Hudepohl, B. Muller, H.-T. Janka, A. Marek and G. G. Raffelt, Phys. Rev. Lett. 104, 251101 (2010) [Erratum-ibid. 105, 249901 (2010)] [arXiv:0912.0260 [astro-ph.SR]].

[83] T. Fischer, S. C. Whitehouse, A. Mezzacappa, F.-K. Thielemann and M. Liebendorfer, Astron. Astrophys. 517, A80 (2010) [arXiv:0908.1871 [astro-ph.HE]].

[84] K. Sumiyoshi, S. Yamada, H. Suzuki and S. Chiba, Phys. Rev. Lett. 97, 091101 (2006) [astro-ph/0608509].

[85] E. O'Connor and C. D. Ott, Astrophys. J. 762, 126 (2013) [arXiv:1207.1100 [astro-ph.HE]].

[86] K. Nakazato, K. Sumiyoshi, H. Suzuki, T. Totani, H. Umeda and S. Yamada, Astrophys. J. Supp. 205, 2 (2013) [arXiv:1210.6841 [astroph.HE]].

[87] K. Nakazato, K. Sumiyoshi, H. Suzuki and S. Yamada, Phys. Rev. D 78, 083014 (2008) [Erratum-ibid. D 79, 069901 (2009)] [arXiv:0810.3734 [astro-ph]].

[88] M. Liebendoerfer, O. E. B. Messer, A. Mezzacappa, S. W. Bruenn, C. Y. Cardall and F. K. Thielemann, Astrophys. J. Suppl. 150, 263 (2004) [astro-ph/0207036].

[89] K. Sumiyoshi, S. Yamada and H. Suzuki, Astrophys. J. 667, 382 (2007) [arXiv:0706.3762 [astro-ph]].

[90] T. Fischer, S. C. Whitehouse, A. Mezzacappa, F.-K. Thielemann and M. Liebendorfer, arXiv:0809.5129 [astro-ph].

[91] K. Sumiyoshi, S. Yamada and H. Suzuki, arXiv:0808.0384 [astro-ph]. 
[92] J. G. Keehn and C. Lunardini, Phys. Rev. D 85, 043011 (2012) [arXiv:1012.1274 [astro-ph.CO]].

[93] S. E. Woosley, and T. A. Weaver, Astrophys. J. Suppl. 101, 181 (1995).

[94] H. Shen, H. Toki, K. Oyamatsu, and K. Sumiyoshi, Nucl. Phys. A, 637, 435 (1998).

[95] J. M. Lattimer and F. D. Swesty, Nucl. Phys. A 535, 331 (1991).

[96] Q. R. Ahmad et al. [SNO Collaboration], Phys. Rev. Lett. 87, 071301 (2001) [nucl-ex/0106015].

[97] P. I. Krastev and S. T. Petcov, Phys. Lett. B 205, 84 (1988).

[98] J. Beringer et al. [Particle Data Group Collaboration], Phys. Rev. D 86, 010001 (2012).

[99] E. K. Akhmedov, C. Lunardini and A. Y. Smirnov, Nucl. Phys. B 643, 339 (2002) [hep-ph/0204091].

[100] A. B. Balantekin, J. Gava and C. Volpe, Phys. Lett. B 662, 396 (2008) [arXiv:0710.3112 [astro-ph]].

[101] H. Duan, G. M. Fuller, J. Carlson and Y. Z. Qian, Phys. Rev. D 74, 105014 (2006) [astro-ph/0606616].

[102] H. Duan, G. M. Fuller, J. Carlson and Y. Z. Qian, Phys. Rev. Lett. 97, 241101 (2006) [astro-ph/0608050].

[103] H. Duan and J. P. Kneller, J. Phys. G 36, 113201 (2009) [arXiv:0904.0974 [astro-ph.HE]].

[104] A. Esteban-Pretel, A. Mirizzi, S. Pastor, R. Tomas, G. G. Raffelt, P. D. Serpico and G. Sigl, Phys. Rev. D 78, 085012 (2008) [arXiv:0807.0659 [astro-ph]].

[105] H. Duan, G. M. Fuller, J. Carlson and Y. Q. Zhong, Phys. Rev. Lett. 99, 241802 (2007) [arXiv:0707.0290 [astro-ph]].

[106] S. Chakraborty, T. Fischer, A. Mirizzi, N. Saviano and R. Tomas, Phys. Rev. Lett. 107, 151101 (2011) [arXiv:1104.4031 [hep-ph]]. 
[107] S. Chakraborty, T. Fischer, A. Mirizzi, N. Saviano and R. Tomas, Phys. Rev. D 84, 025002 (2011) [arXiv:1105.1130 [hep-ph]].

[108] B. Dasgupta, E. P. O'Connor and C. D. Ott, Phys. Rev. D 85, 065008 (2012) [arXiv:1106.1167 [astro-ph.SR]].

[109] L. Wolfenstein, Phys. Rev. D 17, 2369 (1978).

[110] S. P. Mikheev and A. Y. Smirnov, Sov. J. Nucl. Phys. 42, 913 (1985) [Yad. Fiz. 42, 1441 (1985)].

[111] S. P. Mikheev and A. Y. Smirnov, Sov. Phys. JETP 64, 4 (1986) [Zh. Eksp. Teor. Fiz. 91, 7 (1986)] [arXiv:0706.0454 [hep-ph]].

[112] C. Lunardini and A. Y. Smirnov, JCAP 0306, 009 (2003) [hep$\mathrm{ph} / 0302033]$.

[113] R. C. Schirato and G. M. Fuller, astro-ph/0205390.

[114] A. S. Dighe and A. Y. Smirnov, Phys. Rev. D 62, 033007 (2000) [hep$\mathrm{ph} / 9907423]$.

[115] C. Lunardini, Phys. Rev. D 73, 083009 (2006) [hep-ph/0601054].

[116] H. Duan, G. M. Fuller and Y. Z. Qian, Phys. Rev. D 76, 085013 (2007) [arXiv:0706.4293 [astro-ph]].

[117] B. Dasgupta, A. Dighe, A. Mirizzi and G. G. Raffelt, Phys. Rev. D 77, 113007 (2008) [arXiv:0801.1660 [hep-ph]].

[118] B. Dasgupta, A. Dighe, G. G. Raffelt and A. Y. Smirnov, Phys. Rev. Lett. 103, 051105 (2009) [arXiv:0904.3542 [hep-ph]].

[119] J. F. Cherry, J. Carlson, A. Friedland, G. M. Fuller and A. Vlasenko, Phys. Rev. Lett. 108, 261104 (2012) [arXiv:1203.1607 [hep-ph]].

[120] S. Sarikas, I. Tamborra, G. Raffelt, L. Hudepohl and H. T. Janka, Phys. Rev. D 85, 113007 (2012) [arXiv:1204.0971 [hep-ph]].

[121] J. F. Cherry, J. Carlson, A. Friedland, G. M. Fuller and A. Vlasenko, Phys. Rev. D 87, 085037 (2013) [arXiv:1302.1159 [astro-ph.HE]]. 
[122] G. Raffelt, S. Sarikas and D. de Sousa Seixas, Phys. Rev. Lett. 111, no. 9, 091101 (2013) [arXiv:1305.7140 [hep-ph]].

[123] A. Mirizzi, Phys. Rev. D 88, no. 7, 073004 (2013) [arXiv:1308.1402 [hep-ph]].

[124] S. Chakraborty and A. Mirizzi, Phys. Rev. D 90, 033004 (2014) [arXiv:1308.5255 [hep-ph]].

[125] J. P. Kneller and C. Volpe, Phys. Rev. D 82, 123004 (2010) [arXiv:1006.0913 [hep-ph]].

[126] K. Hirata et al. [KAMIOKANDE-II Collaboration], Phys. Rev. Lett. 58, 1490 (1987).

[127] R. M. Bionta, G. Blewitt, C. B. Bratton, D. Casper, A. Ciocio, R. Claus, B. Cortez and M. Crouch et al., Phys. Rev. Lett. 58, 1494 (1987).

[128] C. Lunardini, Phys. Rev. D 75, 073022 (2007) [astro-ph/0612701].

[129] M. S. Malek, UMI-31-06530.

[130] G. Battistoni, A. Ferrari, T. Montaruli and P. R. Sala, Astropart. Phys. 23, 526 (2005).

[131] J. N. Bahcall, A. M. Serenelli and S. Basu, Astrophys. J. 621, L85 (2005) [astro-ph/0412440].

[132] T. Araki et al. [KamLAND Collaboration], Phys. Rev. Lett. 94, 081801 (2005) [hep-ex/0406035].

[133] T. Araki, S. Enomoto, K. Furuno, Y. Gando, K. Ichimura, H. Ikeda, K. Inoue and Y. Kishimoto et al., Nature 436, 499 (2005).

[134] G. Bellini et al. [ Borexino Collaboration], Phys. Lett. B 687, 299 (2010) [arXiv:1003.0284 [hep-ex]].

[135] O. L. G. Peres and A. Y. Smirnov, Phys. Rev. D 79, 113002 (2009) [arXiv:0903.5323 [hep-ph]]. 
[136] Suzuki, Y., 2004, talk at the 2004 Nobel Symposium on Neutrino Physics, Enkoping, Sweden, published in L. Bergstrom, O. Botner, P. Carlson, P. O. Hulth, T. Ohlsson: Neutrino Physics, Physica Scripta, vol. T121, 2005.

[137] M. Smy, private communication.

[138] A. Strumia and F. Vissani, Phys. Lett. B 564, 42 (2003) [astroph/0302055].

[139] A. Rubbia, private communication.

[140] H. K. M. Tanaka, H. Watanabe, Nature Scientific Reports, Volume 4, id. 4708 (2014).

[141] M. Apollonio et al. [CHOOZ Collaboration], Phys. Lett. B 466, 415 (1999) [hep-ex/9907037].

[142] E. N. Alekseev, L. N. Alekseeva, V. I. Volchenko and I. V. Krivosheina, JETP Lett. 45, 589 (1987) [Pisma Zh. Eksp. Teor. Fiz. 45, 461 (1987)].

[143] V. L. Dadykin, G. T. Zatsepin, V. B. Korchagin, P. V. Korchagin, A. S. Malgin, O. G. Ryazhskaya, V. G. Ryasnyi and V. P. Talochkin et al., JETP Lett. 45, 593 (1987) [Pisma Zh. Eksp. Teor. Fiz. 45, 464 (1987)].

[144] M. Selvi [LVD Collaboration], AIP Conf. Proc. 944, 16 (2007).

[145] N. Y. Agafonova, M. Aglietta, P. Antonioli, G. Bari, V. V. Boyarkin, G. Bruno, W. Fulgione and P. Galeotti et al., Astropart. Phys. 27, 254 (2007) [hep-ph/0609305].

[146] S. Amerio et al. [ICARUS Collaboration], Nucl. Instrum. Meth. A 527, 329 (2004).

[147] D. B. Cline, F. Raffaelli and F. Sergiampietri, JINST 1, T09001 (2006) [astro-ph/0604548].

[148] A. Rubbia, J. Phys. Conf. Ser. 171, 012020 (2009) [arXiv:0908.1286 [hep-ph]]. 
[149] K. Eguchi et al. [KamLAND Collaboration], Phys. Rev. Lett. 92, 071301 (2004) [hep-ex/0310047].

[150] B. Aharmim et al. [SNO Collaboration], Phys. Rev. D 70, 093014 (2004) [hep-ex/0407029].

[151] B. Aharmim et al. [SNO Collaboration], Astrophys. J. 653, 1545 (2006) [hep-ex/0607010].

[152] A. Gando, Y. Gando, K. Ichimura, H. Ikeda, K. Inoue, Y. Kibe, Y. Kishimoto and M. Koga et al., Astrophys. J. 745, 193 (2012) [arXiv:1105.3516 [astro-ph.HE]].

[153] C. Lunardini and O. L. G. Peres, JCAP 0808, 033 (2008) [arXiv:0805.4225 [astro-ph]].

[154] G. Bellini et al. [Borexino Collaboration], Phys. Lett. B 696, 191 (2011) [arXiv:1010.0029 [hep-ex]].

[155] T. Marrodan Undagoitia, F. von Feilitzsch, M. Goder-Neff, K. A. Hochmuth, L. Oberauer, W. Potzel and M. Wurm, Prog. Part. Nucl. Phys. 57, 283 (2006) [J. Phys. Conf. Ser. 39, 278 (2006)] [hep$\mathrm{ph} / 0605229]$.

[156] J. F. Beacom, Ann. Rev. Nucl. Part. Sci. 60, 439 (2010) [arXiv:1004.3311 [astro-ph.HE]].

[157] K. Bays, private communication.

[158] B. Jegerlehner, F. Neubig and G. Raffelt, Phys. Rev. D 54, 1194 (1996) [astro-ph/9601111].

[159] K. S. Hirata, T. Kajita, M. Koshiba, M. Nakahata, Y. Oyama, N. Sato, A. Suzuki and M. Takita et al., Phys. Rev. D 38, 448 (1988).

[160] J. N. Bahcall, P. I. Krastev and E. Lisi, Phys. Rev. C 55, 494 (1997) [nucl-ex/9610010].

[161] K. Nakamura, Int. J. Mod. Phys. A 18, 4053 (2003).

[162] M. Diwan, S. H. Kettell, L. Littenberg, W. Marciano, Z. Parsa, N. Samios, S. White and R. Lanou et al., hep-ex/0608023. 
[163] A. Achterberg et al. [IceCube Collaboration], Astropart. Phys. 26, 155 (2006) [astro-ph/0604450].

[164] D. Autiero, J. Aysto, A. Badertscher, L. B. Bezrukov, J. Bouchez, A. Bueno, J. Busto and J. E. Campagne et al., JCAP 0711, 011 (2007) [arXiv:0705.0116 [hep-ph]].

[165] A. Bernstein, M. Bishai, E. Blucher, D. B. Cline, M. V. Diwan, B. Fleming, M. Goodman and Z. J. Hladysz et al., arXiv:0907.4183 [hep-ex].

[166] A. Renshaw et al. [Super-Kamiokande Collaboration], Phys. Rev. Lett. 112, 091805 (2014) [arXiv:1312.5176 [hep-ex]].

[167] M. Ikeda, talk at the Workshop for Neutrino Programs with facilities in Japan, Ibaraki, Japan, available at http://wwwconf.kek.jp/ws_nu_prog_in_jp/

[168] H. Watanabe et al. [Super-Kamiokande Collaboration], Astropart. Phys. 31, 320 (2009) [arXiv:0811.0735 [hep-ex]].

[169] A. Kibayashi [Super-Kamiokande Collaboration], arXiv:0909.5528 [astro-ph.IM].

[170] S. M. Adams, C. S. Kochanek, J. F. Beacom, M. R. Vagins and K. Z. Stanek, Astrophys. J. 778, 164 (2013) [arXiv:1306.0559 [astroph.HE]].

[171] P. Fernandez, talk at ICHEP2014, available at http://ichep2014.es.

[172] M. Vagins, talk at $\mathrm{TeV}$ particle astrophysics 2009, available at http://www-conf.slac.stanford.edu/tevpa09/.

[173] Y. Efremenko, talk at Advances in Neutrino Technology, Manoa, HI, 2009, at http://www.physics.ucdavis.edu/ANT09/.

[174] R. Moellenberg, Diploma thesis, Technische Universität München, 2009.

[175] CERN Courier, February 23 issue, 2015. Available at http://cerncourier.com/cws/article/cern/60150 . 
[176] A. Ereditato and A. Rubbia, Nucl. Phys. Proc. Suppl. 155, 233 (2006) [hep-ph/0510131].

[177] M. Antonello, B. Baibussinov, V. Bellini, H. Bilokon, F. Boffelli, M. Bonesini, E. Calligarich and S. Centro et al., arXiv:1312.7252 [physics.ins-det].

[178] G. L. Raselli [ICARUS Collaboration], Nuovo Cim. C 036, no. 01, 243 (2013).

[179] H. Chen et al. [MicroBooNE Collaboration], FERMILABPROPOSAL-0974.

[180] M. Soderberg [MicroBooNE Collaboration], AIP Conf. Proc. 1189, 83 (2009) [arXiv:0910.3497 [physics.ins-det]].

[181] M. Soderberg [ArgoNeuT Collaboration], arXiv:0910.3433 [physics.insdet].

[182] T. Yang [ArgoNeuT Collaboration], arXiv:1311.2096 [hep-ex].

[183] S. Skadhauge and R. Zukanovich Funchal, JCAP 0704, 014 (2007) [hep-ph/0611194].

[184] H. Minakata, H. Nunokawa, R. Tomas and J. W. F. Valle, JCAP 0812, 006 (2008) [arXiv:0802.1489 [hep-ph]].

[185] L. J. Hall, H. Murayama, M. Papucci and G. Perez, hep-ph/0607109.

[186] S. Palomares-Ruiz and S. Pascoli, Phys. Rev. D 77, 025025 (2008) [arXiv:0710.5420 [astro-ph]].

[187] S. Palomares-Ruiz, Phys. Lett. B 665, 50 (2008) [arXiv:0712.1937 [astro-ph]].

[188] N. Bernal, J. Martn-Albo and S. Palomares-Ruiz, JCAP 1308, 011 (2013) [arXiv:1208.0834 [hep-ph]].

[189] G. Raffelt and T. Rashba, Phys. Atom. Nucl. 73, 609 (2010) [arXiv:0902.4832 [astro-ph.HE]].

[190] Y. Suzuki et al. [TITAND Working Group Collaboration], hepex/0110005. 
[191] M. D. Kistler, H. Yuksel, S. Ando, J. F. Beacom and Y. Suzuki, Phys. Rev. D 83, 123008 (2011) [arXiv:0810.1959 [astro-ph]].

[192] S. Bser, M. Kowalski, L. Schulte, N. L. Strotjohann and M. Voge, arXiv:1304.2553 [astro-ph.IM].

[193] K. A. Hochmuth, F. V. Feilitzsch, B. D. Fields, T. Marrodan Undagoitia, L. Oberauer, W. Potzel, G. G. Raffelt and M. Wurm, Astropart. Phys. 27, 21 (2007) [hep-ph/0509136].

[194] A. Terashima et al. [KamLAND Collaboration], J. Phys. Conf. Ser. 120, 052029 (2008).

[195] A. Gando et al. [KamLAND Collaboration], Phys. Rev. D 88, no. 3, 033001 (2013) [arXiv:1303.4667 [hep-ex]].

[196] J. G. Learned, S. T. Dye and S. Pakvasa, arXiv:0810.4975 [hep-ex].

[197] Supernova Legacy Survey, http://cfht.hawaii.edu/SNLS/.

[198] Dark Energy Survey, http://www.darkenergysurvey.org

[199] Large Synoptic Survey Telescope, http://www.lsst.org

[200] James Webb Space Telescope, http://www.jwst.nasa.gov/.

[201] European Extremely Large Telescope, http://www.eso.org/sci/facilities/eelt/ 